\title{
Strong and Weak Convergence Criteria of Composite Iterative Algorithms for Systems of Generalized Equilibria
}

\author{
Lu-Chuan Ceng, ${ }^{1}$ Cheng-Wen Liao, ${ }^{2}$ Chin-Tzong Pang, \\ Ching-Feng Wen, ${ }^{4}$ and Zhao-Rong Kong ${ }^{5}$ \\ ${ }^{1}$ Department of Mathematics, Shanghai Normal University and Scientific Computing Key Laboratory of Shanghai Universities, \\ Shanghai 200234, China \\ ${ }^{2}$ Department of Food and Beverage Management, Vanung University, Chung-Li 320061, Taiwan \\ ${ }^{3}$ Department of Information Management, Yuan Ze University, Chung-Li 32003, Taiwan \\ ${ }^{4}$ Center for Fundamental Science, Kaohsiung Medical University, Kaohsiung 807, Taiwan \\ ${ }^{5}$ Department of Mathematics, Shanghai Normal University, Shanghai 200234, China
}

Correspondence should be addressed to Chin-Tzong Pang; imctpang@saturn.yzu.edu.tw

Received 20 January 2014; Accepted 12 February 2014; Published 25 March 2014

Academic Editor: Jen-Chih Yao

Copyright (C) 2014 Lu-Chuan Ceng et al. This is an open access article distributed under the Creative Commons Attribution License, which permits unrestricted use, distribution, and reproduction in any medium, provided the original work is properly cited.

We first introduce and analyze one iterative algorithm by using the composite shrinking projection method for finding a solution of the system of generalized equilibria with constraints of several problems: a generalized mixed equilibrium problem, finitely many variational inequalities, and the common fixed point problem of an asymptotically strict pseudocontractive mapping in the intermediate sense and infinitely many nonexpansive mappings in a real Hilbert space. We prove a strong convergence theorem for the iterative algorithm under suitable conditions. On the other hand, we also propose another iterative algorithm involving no shrinking projection method and derive its weak convergence under mild assumptions. Our results improve and extend the corresponding results in the earlier and recent literature.

\section{Introduction}

Let $H$ be a real Hilbert space with inner product $\langle\cdot, \cdot\rangle$ and norm $\|\cdot\|, C$ a nonempty closed convex subset of $H$, and $P_{C}$ the metric projection of $H$ onto $C$. Let $S: C \rightarrow H$ be a nonlinear mapping on $C$. We denote by $\operatorname{Fix}(S)$ the set of fixed points of $S$ and by $\mathbf{R}$ the set of all real numbers. A mapping $V$ is called strongly positive on $H$ if there exists a constant $\bar{\gamma}>0$ such that

$$
\langle V x, x\rangle \geq \bar{\gamma}\|x\|^{2}, \quad \forall x \in H .
$$

A mapping $S: C \rightarrow H$ is called $L$-Lipschitz continuous if there exists a constant $L \geq 0$ such that

$$
\|S x-S y\| \leq L\|x-y\|, \quad \forall x, y \in C .
$$

In particular, if $L=1$ then $S$ is called a nonexpansive mapping; if $L \in[0,1)$ then $A$ is called a contraction.

Let $A: C \rightarrow H$ be a nonlinear mapping on $C$. We consider the following variational inequality problem (VIP): find a point $x \in C$ such that

$$
\langle A x, y-x\rangle \geq 0, \quad \forall y \in C .
$$

The solution set of VIP (3) is denoted by VI $(C, A)$.

The VIP (3) was first discussed by Lions [1] and now is well known; there are a lot of different approaches towards solving VIP (3) in finite-dimensional and infinite-dimensional spaces, and the research is intensively continued. The VIP (3) has many applications in computational mathematics, mathematical physics, operations research, mathematical economics, optimization theory, and other fields; see, for example, [2-5]. It is well known that if $A$ is strongly monotone 
and Lipschitz-continuous mapping on C, then VIP (3) has a unique solution. Not only are the existence and uniqueness of solutions important topics in the study of VIP (3), but also how to actually find a solution of VIP (3) is important. Up to now, there have been many iterative algorithms in the literature, for finding approximate solutions of VIP (3) and its extended versions; see, for example, [6-11].

In 1976, Korpelevič [12] proposed an iterative algorithm for solving the VIP (3) in Euclidean space $\mathbf{R}^{n}$ :

$$
\begin{gathered}
y_{n}=P_{C}\left(x_{n}-\tau A x_{n}\right), \\
x_{n+1}=P_{C}\left(x_{n}-\tau A y_{n}\right), \quad \forall n \geq 0,
\end{gathered}
$$

with $\tau>0$ a given number, which is known as the extragradient method. The literature on the VIP is vast and Korpelevich's extragradient method has received great attention given by many authors, who improved it in various ways; see, for example, $[10,11,13-23]$ and references therein, to name but a few.

Let $\varphi: C \rightarrow \mathbf{R}$ be a real-valued function, $A: H \rightarrow H$ a nonlinear mapping, and $\Theta: C \times C \rightarrow \mathbf{R}$ a bifunction. In 2008, Peng and Yao [18] introduced the following generalized mixed equilibrium problem (GMEP) of finding $x \in C$ such that

$$
\Theta(x, y)+\varphi(y)-\varphi(x)+\langle A x, y-x\rangle \geq 0, \quad \forall y \in C .
$$

We denote the set of solutions of GMEP (5) by $\operatorname{GMEP}(\Theta, \varphi, A)$. The GMEP (5) is very general in the sense that it includes, as special cases, optimization problems, variational inequalities, minimax problems, Nash equilibrium problems in noncooperative games, and others. The GMEP is further considered and studied; see, for example, [20, 23-28].

If $\varphi=0$, then GMEP (5) reduces to the generalized equilibrium problem (GEP) which is to find $x \in C$ such that

$$
\Theta(x, y)+\langle A x, y-x\rangle \geq 0, \quad \forall y \in C .
$$

It is introduced and studied by S. Takahashi and W. Takahashi [29]. The set of solutions of GEP is denoted by $\operatorname{GEP}(\Theta, A)$.

If $A=0$, then GMEP (5) reduces to the mixed equilibrium problem (MEP) which is to find $x \in C$ such that

$$
\Theta(x, y)+\varphi(y)-\varphi(x) \geq 0, \quad \forall y \in C .
$$

It is considered and studied in [30-32]. The set of solutions of MEP is denoted by $\operatorname{MEP}(\Theta, \varphi)$.

If $\varphi=0, A=0$, then GMEP (5) reduces to the equilibrium problem (EP) which is to find $x \in C$ such that

$$
\Theta(x, y) \geq 0, \quad \forall y \in C .
$$

It is considered and studied in $[33,34]$. The set of solutions of EP is denoted by $\operatorname{EP}(\Theta)$. It is worth mentioning that the EP is a unified model of several problems, namely, variational inequality problems, optimization problems, saddle point problems, complementarity problems, fixed point problems, Nash equilibrium problems, and so forth.
Throughout this paper, we assume as in [18] that $\Theta: C \times$ $C \rightarrow \mathbf{R}$ is a bifunction satisfying conditions (H1)-(H4) and $\varphi: C \rightarrow \mathbf{R}$ is a lower semicontinuous and convex function with restriction $(\mathrm{H} 5)$, where

(H1) $\Theta(x, x)=0$ for all $x \in C$;

$(\mathrm{H} 2) \Theta$ is monotone; that is, $\Theta(x, y)+\Theta(y, x) \leq 0$ for any $x, y \in C$;

(H3) $\Theta$ is upper-hemicontinuous; that is, for each $x, y, z \in$ C,

$$
\limsup _{t \rightarrow 0^{+}} \Theta(t z+(1-t) x, y) \leq \Theta(x, y)
$$

(H4) $\Theta(x, \cdot)$ is convex and lower semicontinuous for each $x \in C$;

(H5) for each $x \in H$ and $r>0$ there exists a bounded subset $D_{x} \subset C$ and $y_{x} \in C$ such that, for any $z \in C \backslash D_{x}$,

$$
\Theta\left(z, y_{x}\right)+\varphi\left(y_{x}\right)-\varphi(z)+\frac{1}{r}\left\langle y_{x}-z, z-x\right\rangle<0 .
$$

Given a positive number $r>0$, let $S_{r}^{(\Theta, \varphi)}: H \rightarrow C$ be the solution set of the auxiliary mixed equilibrium problem; that is, for each $x \in H$,

$$
\begin{aligned}
S_{r}^{(\Theta, \varphi)}(x):= & \{y \in C: \Theta(y, z)+\varphi(z)-\varphi(y) \\
& \left.+\frac{1}{r}\left\langle K^{\prime}(y)-K^{\prime}(x), z-y\right\rangle \geq 0, \forall z \in C\right\} .
\end{aligned}
$$

In particular, whenever $K(x)=(1 / 2)\|x\|^{2}, \forall x \in H, S_{r}^{(\Theta, \varphi)}$ is rewritten as $T_{r}^{(\Theta, \varphi)}$.

Let $\Theta_{1}, \Theta_{2}: C \times C \rightarrow \mathbf{R}$ be two bifunctions and $A_{1}, A_{2}$ : $C \rightarrow H$ two nonlinear mappings. Consider the following system of generalized equilibrium problems (SGEP): find $\left(x^{*}, y^{*}\right) \in C \times C$ such that

$$
\begin{aligned}
& \Theta_{1}\left(x^{*}, x\right)+\left\langle A_{1} y^{*}, x-x^{*}\right\rangle \\
& +\frac{1}{v_{1}}\left\langle x^{*}-y^{*}, x-x^{*}\right\rangle \geq 0, \quad \forall x \in C, \\
& \Theta_{2}\left(y^{*}, y\right)+\left\langle A_{2} x^{*}, y-y^{*}\right\rangle \\
& +\frac{1}{v_{2}}\left\langle y^{*}-x^{*}, y-y^{*}\right\rangle \geq 0, \quad \forall y \in C,
\end{aligned}
$$

where $v_{1}>0$ and $v_{2}>0$ are two constants. It is introduced and studied in [19]. Whenever $\Theta_{1} \equiv \Theta_{2} \equiv 0$, the SGEP reduces to a system of variational inequalities, which is considered and studied in [13]. It is worth mentioning that the system of variational inequalities is a tool to solve the Nash equilibrium problem for noncooperative games.

In 2010, Ceng and Yao [19] transformed the SGEP into a fixed point problem in the following way. 
Proposition CY (see [19]). Let $\Theta_{1}, \Theta_{2}: C \times C \rightarrow \mathbf{R}$ be two bifunctions satisfying conditions (H1)-(H4) and let $A_{k}$ : $C \rightarrow H$ be $\zeta_{k}$-inverse strongly monotone for $k=1,2$. Let $\nu_{k} \in\left(0,2 \zeta_{k}\right)$ for $k=1,2$. Then $\left(x^{*}, y^{*}\right) \in C \times C$ is a solution of SGEP (12) if and only if $x^{*}$ is a fixed point of the mapping $G: C \rightarrow C$ defined by $G=T_{v_{1}}^{\Theta_{1}}\left(I-v_{1} A_{1}\right) T_{v_{2}}^{\Theta_{2}}\left(I-v_{2} A_{2}\right)$, where $y^{*}=T_{\nu_{2}}^{\Theta_{2}}\left(I-v_{2} A_{2}\right) x^{*}$. Here, one denotes the fixed point set of $G$ by $\operatorname{SGEP}(G)$.

Let $\left\{T_{n}\right\}_{n=1}^{\infty}$ be an infinite family of nonexpansive mappings on $H$ and $\left\{\lambda_{n}\right\}_{n=1}^{\infty}$ a sequence of nonnegative numbers in $[0,1]$. For any $n \geq 1$, define a mapping $W_{n}$ on $H$ as follows:

$$
\begin{gathered}
U_{n, n+1}=I, \\
U_{n, n}=\lambda_{n} T_{n} U_{n, n+1}+\left(1-\lambda_{n}\right) I, \\
U_{n, n-1}=\lambda_{n-1} T_{n-1} U_{n, n}+\left(1-\lambda_{n-1}\right) I, \\
\vdots \\
U_{n, k}=\lambda_{k} T_{k} U_{n, k+1}+\left(1-\lambda_{k}\right) I, \\
U_{n, k-1}=\lambda_{k-1} T_{k-1} U_{n, k}+\left(1-\lambda_{k-1}\right) I, \\
\vdots \\
U_{n, 2}=\lambda_{2} T_{2} U_{n, 3}+\left(1-\lambda_{2}\right) I, \\
W_{n}=U_{n, 1}=\lambda_{1} T_{1} U_{n, 2}+\left(1-\lambda_{1}\right) I .
\end{gathered}
$$

Such a mapping $W_{n}$ is called the $W$-mapping generated by $T_{n}, T_{n-1}, \ldots, T_{1}$ and $\lambda_{n}, \lambda_{n-1}, \ldots, \lambda_{1}$.

In 2011, for the case where $C=H$, Yao et al. [25] proposed the following hybrid iterative algorithm:

$$
\begin{gathered}
\Theta\left(y_{n}, z\right)+\varphi(z)-\varphi\left(y_{n}\right) \\
+\frac{1}{r}\left\langle K^{\prime}\left(y_{n}\right)-K^{\prime}\left(x_{n}\right), z-y_{n}\right\rangle \geq 0, \quad z \in H, \\
x_{n+1}=\alpha_{n}\left(u+\gamma f\left(x_{n}\right)\right)+\beta_{n} x_{n} \\
+\left(\left(1-\beta_{n}\right) I-\alpha_{n}(I+\mu V)\right) W_{n} y_{n}, \quad \forall n \geq 1,
\end{gathered}
$$

where $f: H \rightarrow H$ is a contraction, $K: H \rightarrow \mathbf{R}$ is differentiable and strongly convex, $\left\{\alpha_{n}\right\},\left\{\beta_{n}\right\} \subset(0,1)$, and $x_{0}, u \in H$ are given, for finding a common element of the set $\operatorname{MEP}(\Theta, \varphi)$ and the fixed point set $\cap_{n=1}^{\infty} \operatorname{Fix}\left(T_{n}\right)$ of an infinite family of nonexpansive mappings $\left\{T_{n}\right\}_{n=1}^{\infty}$ on $H$. They proved the strong convergence of the sequence generated by the hybrid iterative algorithm (14) to a point $x^{*} \in \Omega:=\cap_{n=1}^{\infty} \operatorname{Fix}\left(T_{n}\right) \cap \operatorname{MEP}(\Theta, \varphi)$ under some appropriate conditions. This point $x^{*}$ also solves the following optimization problem:

$$
\min _{x \in \Omega} \frac{\mu}{2}\langle V x, x\rangle+\frac{1}{2}\|x-u\|^{2}-h(x),
$$

where $h: H \rightarrow \mathbf{R}$ is the potential function of $\gamma f$.
Let $f: H \rightarrow H$ be a contraction and $V$ a strongly positive bounded linear operator on $H$. Assume that $\varphi$ : $H \rightarrow \mathbf{R}$ is a lower semicontinuous and convex functional, that $\Theta, \Theta_{1}, \Theta_{2}: H \times H \rightarrow \mathbf{R}$ satisfy conditions (H1)(H4), and that $A, A_{1}, A_{2}: H \rightarrow H$ are inverse strongly monotone. Let the mapping $G$ be defined as in Proposition CY. Very recently, Ceng et al. [20] introduced the following hybrid extragradient-like iterative algorithm:

$$
\begin{gathered}
z_{n}=S_{r_{n}}^{(\Theta, \varphi)}\left(x_{n}-r_{n} A x_{n}\right), \\
x_{n+1}=\alpha_{n}\left(u+\gamma f\left(x_{n}\right)\right)+\beta_{n} x_{n} \\
+\left(\left(1-\beta_{n}\right) I-\alpha_{n}(I+\mu V)\right) W_{n} G z_{n}, \quad \forall n \geq 0,
\end{gathered}
$$

for finding a common solution of GMEP (5), SGEP (12), and the fixed point problem of an infinite family of nonexpansive mappings $\left\{T_{n}\right\}_{n=1}^{\infty}$ on $H$, where $\left\{r_{n}\right\} \subset(0, \infty),\left\{\alpha_{n}\right\},\left\{\beta_{n}\right\} \subset$ $(0,1), v_{k} \in\left(0,2 \zeta_{k}\right), k=1,2$, and $x_{0}, u \in H$ are given. The authors proved the strong convergence of the sequence generated by the hybrid iterative algorithm (16) to a point $x^{*} \in \Omega:=\cap_{n=1}^{\infty} \operatorname{Fix}\left(T_{n}\right) \cap \operatorname{GMEP}(\Theta, \varphi, A) \cap \operatorname{SGEP}(G)$ under some suitable conditions. This point $x^{*}$ also solves the following optimization problem:

$$
\min _{x \in \Omega} \frac{\mu}{2}\langle V x, x\rangle+\frac{1}{2}\|x-u\|^{2}-h(x),
$$

where $h: H \rightarrow \mathbf{R}$ is the potential function of $\gamma f$.

On the other hand, let $C$ be a nonempty subset of a normed space $X$. A mapping $S: C \rightarrow C$ is called uniformly Lipschitzian if there exists a constant $\mathscr{L}>0$ such that

$$
\left\|S^{n} x-S^{n} y\right\| \leq \mathscr{L}\|x-y\|, \quad \forall n \geq 1, \forall x, y \in C .
$$

Recently, Kim and $\mathrm{Xu}$ [35] introduced the concept of asymptotically $k$-strict pseudocontractive mappings in a Hilbert space as below.

Definition 1. Let $C$ be a nonempty subset of a Hilbert space $H$. A mapping $S: C \rightarrow C$ is said to be an asymptotically $k$ strict pseudocontractive mapping with sequence $\left\{\gamma_{n}\right\}$ if there exist a constant $k \in[0,1)$ and a sequence $\left\{\gamma_{n}\right\}$ in $[0, \infty)$ with $\lim _{n \rightarrow \infty} \gamma_{n}=0$ such that

$$
\begin{array}{r}
\left\|S^{n} x-S^{n} y\right\|^{2} \leq\left(1+\gamma_{n}\right)\|x-y\|^{2} \\
+k\left\|x-S^{n} x-\left(y-S^{n} y\right)\right\|^{2}, \\
\forall n \geq 1, \quad \forall x, y \in C .
\end{array}
$$

They studied weak and strong convergence theorems for this class of mappings. It is important to note that every asymptotically $k$-strict pseudocontractive mapping with sequence $\left\{\gamma_{n}\right\}$ is a uniformly $\mathscr{L}$-Lipschitzian mapping with $\mathscr{L}=\sup \left\{\left(k+\sqrt{1+(1-k) \gamma_{n}}\right) /(1+k): n \geq 1\right\}$. 
Subsequently, Sahu et al. [36] considered the concept of asymptotically $k$-strict pseudocontractive mappings in the intermediate sense, which are not necessarily Lipschitzian.

Definition 2. Let $C$ be a nonempty subset of a Hilbert space $H$. A mapping $S: C \rightarrow C$ is said to be an asymptotically $k$ strict pseudocontractive mapping in the intermediate sense with sequence $\left\{\gamma_{n}\right\}$ if there exist a constant $k \in[0,1)$ and a sequence $\left\{\gamma_{n}\right\}$ in $[0, \infty)$ with $\lim _{n \rightarrow \infty} \gamma_{n}=0$ such that

$$
\begin{array}{r}
\limsup _{n \rightarrow \infty} \sup _{x, y \in C}\left(\left\|S^{n} x-S^{n} y\right\|^{2}-\left(1+\gamma_{n}\right)\|x-y\|^{2}\right. \\
\left.-k\left\|x-S^{n} x-\left(y-S^{n} y\right)\right\|^{2}\right) \leq 0 .
\end{array}
$$

Put $c_{n}:=\max \left\{0, \sup _{x, y \in C}\left(\left\|S^{n} x-S^{n} y\right\|^{2}-\left(1+\gamma_{n}\right)\|x-y\|^{2}-\right.\right.$ $\left.\left.k\left\|x-S^{n} x-\left(y-S^{n} y\right)\right\|^{2}\right)\right\}$. Then $c_{n} \geq 0(\forall n \geq 1), c_{n} \rightarrow$ $0(n \rightarrow \infty)$, and (13) reduces to the relation

$$
\begin{aligned}
&\left\|S^{n} x-S^{n} y\right\|^{2} \leq\left(1+\gamma_{n}\right)\|x-y\|^{2} \\
&+k\left\|x-S^{n} x-\left(y-S^{n} y\right)\right\|^{2}+c_{n}, \\
& \forall n \geq 1, \forall x, y \in C .
\end{aligned}
$$

Whenever $c_{n}=0$ for all $n \geq 1$ in (21) then $S$ is an asymptotically $k$-strict pseudocontractive mapping with sequence $\left\{\gamma_{n}\right\}$. In 2009, Sahu et al. [36] derived the weak and strong convergence of the modified Mann iteration processes for an asymptotically $k$-strict pseudocontractive mapping in the intermediate sense with sequence $\left\{\gamma_{n}\right\}$. More precisely, they first established one weak convergence theorem for the following iterative scheme:

$$
\begin{gathered}
x_{1}=x \in C \text { chosen arbitrarily, } \\
x_{n+1}=\left(1-\alpha_{n}\right) x_{n}+\alpha_{n} S^{n} x_{n}, \quad \forall n \geq 1,
\end{gathered}
$$

where $0<\delta \leq \alpha_{n} \leq 1-k-\delta, \sum_{n=1}^{\infty} \alpha_{n} c_{n}<\infty$, and $\sum_{n=1}^{\infty} \gamma_{n}<$ $\infty$, and then obtained another strong convergence theorem for the following iterative scheme:

$$
\begin{aligned}
x_{1} & =x \in C \text { chosen arbitrary, } \\
y_{n} & =\left(1-\alpha_{n}\right) x_{n}+\alpha_{n} S^{n} x_{n}, \\
C_{n} & =\left\{z \in C:\left\|y_{n}-z\right\|^{2} \leq\left\|x_{n}-z\right\|^{2}+\theta_{n}\right\}, \\
Q_{n} & =\left\{z \in C:\left\langle x_{n}-z, x-x_{n}\right\rangle \geq 0\right\}, \\
x_{n+1} & =P_{C_{n} \cap Q_{n}} x, \quad \forall n \geq 1,
\end{aligned}
$$

where $0<\delta \leq \alpha_{n} \leq 1-k, \theta_{n}=c_{n}+\gamma_{n} \Delta_{n}$, and $\Delta_{n}=\sup \left\{\left\|x_{n}-z\right\|^{2}: z \in \operatorname{Fix}(S)\right\}<\infty$. Subsequently, the above iterative schemes are extended to develop new iterative algorithms for finding a common solution of the VIP and the fixed point problem of an asymptotically strict pseudocontractive mapping in the intermediate sense; see, for example, $[10,22]$.

In 2009, Yao et al. [30] proposed and analyzed iterative algorithms for finding a common element of the set of fixed points of an asymptotically $k$-strict pseudocontraction and the set of solutions of a mixed equilibrium problem in a real Hilbert space. Very recently, motivated by Yao et al. [30], Cai and $\mathrm{Bu}[26]$ introduced and analyzed the following iterative algorithm by the hybrid shrinking projection method:

$$
\begin{gathered}
\text { pick any } x_{0} \in H, \\
\text { set } C_{1}=C, \quad x_{1}=P_{C_{1}} x_{0}, \\
u_{n}=T_{r_{M, n}}^{\left(\Theta_{M}, \varphi_{M}\right)}\left(I-r_{M, n} A_{M}\right) T_{r_{M-1, n}}^{\left(\Theta_{M-1}, \varphi_{M-1}\right)} \\
\times\left(I-r_{M-1, n} A_{M-1}\right) \cdots T_{\left.r_{1, n}, \varphi_{1}\right)}^{(\Theta}\left(I-r_{1, n} A_{1}\right) x_{n}, \\
z_{n}=P_{C}\left(I-\lambda_{N, n} B_{N}\right) \\
\times P_{C}\left(I-\lambda_{N-1, n} B_{N-1}\right) \cdots P_{C}\left(I-\lambda_{2, n} B_{2}\right) \\
\times P_{C}\left(I-\lambda_{1, n} B_{1}\right) u_{n}, \\
k_{n}=\delta_{n} z_{n}+\left(1-\delta_{n}\right) S^{n} z_{n}, \\
y_{n}=\left(1-\alpha_{n}\right) x_{n}+\alpha_{n} k_{n}, \\
C_{n+1}=\left\{z \in C_{n}:\left\|y_{n}-z\right\|^{2} \leq\left\|x_{n}-z\right\|^{2}+\theta_{n}\right\}, \\
x_{n+1}=P_{C_{n+1}} x_{0}, \quad \forall n \geq 0,
\end{gathered}
$$

for finding a common element of the set $\cap_{k=1}^{M} \operatorname{GMEP}\left(\Theta_{k}, \varphi_{k}, A_{k}\right)$ of solutions of finitely many generalized mixed equilibrium problems, the set $\cap_{i=1}^{N} \mathrm{VI}\left(C, B_{i}\right)$ of solutions of finitely many variational inequalities for inverse strong monotone mappings $\left\{B_{i}\right\}_{i=1}^{N}$, and the set $\operatorname{Fix}(S)$ of fixed points of an asymptotically $k$-strict pseudocontractive mapping $S$ in the intermediate sense (provided that $\Omega=\bigcap_{k=1}^{M} \operatorname{GMEP}\left(\Theta_{k}, \varphi_{k}, A_{k}\right) \cap \cap_{i=1}^{N} \operatorname{VI}\left(C, B_{i}\right) \cap \operatorname{Fix}(S)$ is nonempty and bounded), where $\theta_{n}=\gamma_{n} \Delta_{n}^{2}+c_{n}, \Delta_{n}=$ $\sup \left\{\left\|x_{n}-p\right\|: p \in \Omega\right\}<\infty,\left\{\lambda_{i, n}\right\} \subset\left[a_{i}, b_{i}\right] \subset\left(0,2 \eta_{i}\right),\left\{r_{k, n}\right\} \subset$ $\left[e_{k}, f_{k}\right] \subset\left(0,2 \mu_{k}\right), i \in\{1,2, \ldots, N\}, k \in\{1,2, \ldots, M\}$. It was proven in [26] that under appropriate conditions $\left\{x_{n}\right\}$ converge strongly to $P_{\Omega} x_{0}$.

Motivated and inspired by the above facts, we first introduce and analyze one iterative algorithm by using a composite shrinking projection method for finding a solution of the system of generalized equilibria with constraints of several problems: a generalized mixed equilibrium problem, finitely many variational inequalities, and the common fixed point problem of an asymptotically strict pseudocontractive mapping in the intermediate sense and infinitely many nonexpansive mappings in a real Hilbert space. We prove strong convergence theorem for the iterative algorithm under suitable conditions. On the other hand, we also propose another iterative algorithm involving no shrinking projection method and derive its weak convergence under mild assumptions. Our results improve and extend the corresponding results in the earlier and recent literature. 


\section{Preliminaries}

Let $H$ be a real Hilbert space whose inner product and norm are denoted by $\langle\cdot, \cdot\rangle$ and $\|\cdot\|$, respectively. Let $C$ be a nonempty closed convex subset of $H$. We use the notations $x_{n} \rightarrow x$ and $x_{n} \rightarrow x$ to indicate the weak convergence of $\left\{x_{n}\right\}$ to $x$ and the strong convergence of $\left\{x_{n}\right\}$ to $x$, respectively. Moreover, we use $\omega_{w}\left(x_{n}\right)$ to denote the weak $\omega$-limit set of $\left\{x_{n}\right\}$; that is,

$$
\begin{aligned}
\omega_{w}\left(x_{n}\right):=\left\{x \in H: x_{n_{i}} \rightarrow x\right. \text { for some } \\
\text { subsequence } \left.\left\{x_{n_{i}}\right\} \text { of }\left\{x_{n}\right\}\right\} .
\end{aligned}
$$

Definition 3. A mapping $A: C \rightarrow H$ is called

(i) monotone if

$$
\langle A x-A y, x-y\rangle \geq 0, \quad \forall x, y \in C
$$

(ii) $\eta$-strongly monotone if there exists a constant $\eta>0$ such that

$$
\langle A x-A y, x-y\rangle \geq \eta\|x-y\|^{2}, \quad \forall x, y \in C ;
$$

(iii) $\zeta$-inverse strongly monotone if there exists a constant $\zeta>0$ such that

$$
\langle A x-A y, x-y\rangle \geq \zeta\|A x-A y\|^{2}, \quad \forall x, y \in C .
$$

It is easy to see that the projection $P_{C}$ is 1-inverse strongly monotone. The inverse strongly monotone (also referred to as cocoercive) operators have been applied widely in solving practical problems in various fields.

Definition 4. A differentiable function $K: H \rightarrow \mathbf{R}$ is called

(i) convex if

$$
K(y)-K(x) \geq\left\langle K^{\prime}(x), y-x\right\rangle, \quad \forall x, y \in H,
$$

where $K^{\prime}(x)$ is the Fréchet derivative of $K$ at $x$;

(ii) strongly convex if there exists a constant $\sigma>0$ such that

$$
K(y)-K(x)-\left\langle K^{\prime}(x), y-x\right\rangle \geq \frac{\sigma}{2}\|x-y\|^{2}, \quad \forall x, y \in H .
$$

It is easy to see that if $K: H \rightarrow \mathbf{R}$ is a differentiable strongly convex function with constant $\sigma>0$ then $K^{\prime}: H \rightarrow$ $H$ is strongly monotone with constant $\sigma>0$.

The metric (or nearest point) projection from $H$ onto $C$ is the mapping $P_{C}: H \rightarrow C$ which assigns to each point $x \in H$ the unique point $P_{C} x \in C$ satisfying the property

$$
\left\|x-P_{C} x\right\|=\inf _{y \in C}\|x-y\|=: d(x, C) .
$$

Some important properties of projections are gathered in the following proposition.
Proposition 5. For given $x \in H$ and $z \in C$,

(i) $z=P_{C} x \Leftrightarrow\langle x-z, y-z\rangle \leq 0, \forall y \in C$;

(ii) $z=P_{C} x \Leftrightarrow\|x-z\|^{2} \leq\|x-y\|^{2}-\|y-z\|^{2}, \forall y \in C$;

(iii) $\left\langle P_{C} x-P_{C} y, x-y\right\rangle \geq\left\|P_{C} x-P_{C} y\right\|^{2}, \forall y \in H$. (This implies that $P_{C}$ is nonexpansive and monotone.)

By using the technique of [32], we can readily obtain the following elementary result.

Proposition 6 (see [20, Lemma 1 and Proposition 1]). Let $C$ be a nonempty closed convex subset of a real Hilbert space $H$ and let $\varphi: C \rightarrow \mathbf{R}$ be a lower semicontinuous and convex function. Let $\Theta: C \times C \rightarrow \mathbf{R}$ be a bifunction satisfying the conditions (H1)-(H4). Assume that

(i) $K: H \rightarrow \mathbf{R}$ is strongly convex with constant $\sigma>0$ and the function $x \mapsto\left\langle y-x, K^{\prime}(x)\right\rangle$ is weakly upper semicontinuous for each $y \in H$;

(ii) for each $x \in H$ and $r>0$ there exists a bounded subset $D_{x} \subset C$ and $y_{x} \in C$ such that, for any $z \in C \backslash D_{x}$,

$$
\begin{aligned}
& \Theta\left(z, y_{x}\right)+\varphi\left(y_{x}\right)-\varphi(z) \\
& +\frac{1}{r}\left\langle K^{\prime}(z)-K^{\prime}(x), y_{x}-z\right\rangle<0 .
\end{aligned}
$$

Then the following hold:

(a) for each $x \in H, S_{r}^{(\Theta, \varphi)}(x) \neq \emptyset$;

(b) $S_{r}^{(\Theta, \varphi)}$ is single valued;

(c) $S_{r}^{(\Theta, \varphi)}$ is nonexpansive if $K^{\prime}$ is Lipschitz continuous with constant $v>0$ and

$$
\begin{array}{r}
\left\langle K^{\prime}\left(x_{1}\right)-K^{\prime}\left(x_{2}\right), u_{1}-u_{2}\right\rangle \\
\leq\left\langle K^{\prime}\left(u_{1}\right)-K^{\prime}\left(u_{2}\right), u_{1}-u_{2}\right\rangle, \\
\forall\left(x_{1}, x_{2}\right) \in H \times H,
\end{array}
$$

where $u_{i}=S_{r}^{(\Theta, \varphi)}\left(x_{i}\right)$ for $i=1,2$;

(d) for all $s, t>0$ and $x \in H$,

$$
\begin{aligned}
& \left\langle K^{\prime}\left(S_{s}^{(\Theta, \varphi)} x\right)-K^{\prime}\left(S_{t}^{(\Theta, \varphi)} x\right), S_{s}^{(\Theta, \varphi)} x-S_{t}^{(\Theta, \varphi)} x\right\rangle \\
& \quad \leq \frac{s-t}{s}\left\langle K^{\prime}\left(S_{s}^{(\Theta, \varphi)} x\right)-K^{\prime}(x), S_{s}^{(\Theta, \varphi)} x-S_{t}^{(\Theta, \varphi)} x\right\rangle ;
\end{aligned}
$$

(e) $\operatorname{Fix}\left(S_{r}^{(\Theta, \varphi)}\right)=\operatorname{MEP}(\Theta, \varphi)$;

(f) $\operatorname{MEP}(\Theta, \varphi)$ is closed and convex.

Remark 7. In Proposition 6, whenever $\Theta: C \times C \rightarrow \mathbf{R}$ is a bifunction satisfying the conditions $(\mathrm{H} 1)-(\mathrm{H} 4)$ and $K(x)=$ $(1 / 2)\|x\|^{2}, \forall x \in H$, we have, for any $x, y \in H$,

$$
\left\|S_{r}^{(\Theta, \varphi)} x-S_{r}^{(\Theta, \varphi)} y\right\|^{2} \leq\left\langle S_{r}^{(\Theta, \varphi)} x-S_{r}^{(\Theta, \varphi)} y, x-y\right\rangle
$$


$\left(S_{r}^{(\Theta, \varphi)}\right.$ is firmly nonexpansive $)$ and

$$
\begin{array}{r}
\left\|S_{s}^{(\Theta, \varphi)} x-S_{t}^{(\Theta, \varphi)} x\right\| \leq \frac{|s-t|}{s}\left\|S_{s}^{(\Theta, \varphi)} x-x\right\|, \\
\forall s, t>0, x \in H .
\end{array}
$$

In this case, $S_{r}^{(\Theta, \varphi)}$ is rewritten as $T_{r}^{(\Theta, \varphi)}$. If, in addition, $\varphi \equiv 0$, then $T_{r}^{(\Theta, \varphi)}$ is rewritten as $T_{r}^{\Theta}$; see [19, Lemma 2.1] for more details.

We need some facts and tools in a real Hilbert space $H$ which are listed as lemmas below.

Lemma 8. Let $X$ be a real inner product space. Then the following inequality holds:

$$
\|x+y\|^{2} \leq\|x\|^{2}+2\langle y, x+y\rangle, \quad \forall x, y \in X .
$$

Lemma 9. Let $H$ be a real Hilbert space. Then the following hold:

(a) $\|x-y\|^{2}=\|x\|^{2}-\|y\|^{2}-2\langle x-y, y\rangle$ for all $x, y \in H$;

(b) $\|\lambda x+\mu y\|^{2}=\lambda\|x\|^{2}+\mu\|y\|^{2}-\lambda \mu\|x-y\|^{2}$ for all $x, y \in$ $H$ and $\lambda, \mu \in[0,1]$ with $\lambda+\mu=1$;

(c) if $\left\{x_{n}\right\}$ is a sequence in $H$ such that $x_{n} \rightarrow x$, it follows that

$\limsup _{n \rightarrow \infty}\left\|x_{n}-y\right\|^{2}=\limsup _{n \rightarrow \infty}\left\|x_{n}-x\right\|^{2}+\|x-y\|^{2}, \quad \forall y \in H$.

We have the following crucial lemmas concerning the $W$ mappings defined by (13).

Lemma 10 (see [37, Lemma 3.2]). Let $\left\{T_{n}\right\}_{n=1}^{\infty}$ be a sequence of nonexpansive self-mappings on $H$ such that $\cap_{n=1}^{\infty} \operatorname{Fix}\left(T_{n}\right) \neq \emptyset$ and let $\left\{\lambda_{n}\right\}$ be a sequence in $(0, b]$ for some $b \in(0,1)$. Then, for every $x \in H$ and $k \geq 1$ the limit $\lim _{n \rightarrow \infty} U_{n, k} x$ exists, where $U_{n, k}$ is defined by (13).

Lemma 11 (see [37, Lemma 3.3]). Let $\left\{T_{n}\right\}_{n=1}^{\infty}$ be a sequence of nonexpansive self-mappings on $H$ such that $\cap_{n=1}^{\infty} \operatorname{Fix}\left(T_{n}\right) \neq \emptyset$, and let $\left\{\lambda_{n}\right\}$ be a sequence in $(0, b]$ for some $b \in(0,1)$. Then $\operatorname{Fix}(W)=\bigcap_{n=1}^{\infty} \operatorname{Fix}\left(T_{n}\right)$.

Lemma 12 (see [38, Demiclosedness principle]). Let $C$ be a nonempty closed convex subset of a real Hilbert space H. Let T be a nonexpansive self-mapping on $C$. Then $I-T$ is demiclosed. That is, whenever $\left\{x_{n}\right\}$ is a sequence in $C$ weakly converging to some $x \in C$ and the sequence $\left\{(I-T) x_{n}\right\}$ strongly converges to some $y$, it follows that $(I-T) x=y$. Here $I$ is the identity operator of $H$.

Lemma 13. Let $A: C \rightarrow H$ be a monotone mapping. In the context of the variational inequality problem the characterization of the projection (see Proposition 5(i)) implies

$$
u \in \mathrm{VI}(C, A) \Longleftrightarrow u=P_{C}(u-\lambda A u), \quad \lambda>0 .
$$

Lemma 14 (see [36, Lemma 2.5]). Let $H$ be a real Hilbert space. Given a nonempty closed convex subset of $H$ and points $x, y, z \in H$ and given also a real number $a \in \mathbf{R}$, the set

$$
\left\{v \in C:\|y-v\|^{2} \leq\|x-v\|^{2}+\langle z, v\rangle+a\right\}
$$

is convex (and closed).

Recall that a set-valued mapping $T: D(T) \subset H \rightarrow 2^{H}$ is called monotone if, for all $x, y \in D(T), f \in T x$ and $g \in T y$ imply

$$
\langle f-g, x-y\rangle \geq 0 \text {. }
$$

A set-valued mapping $T$ is called maximal monotone if $T$ is monotone and $(I+\lambda T) D(T)=H$ for each $\lambda>0$, where $I$ is the identity mapping of $H$. We denote by $G(T)$ the graph of $T$. It is known that a monotone mapping $T$ is maximal if and only if, for $(x, f) \in H \times H,\langle f-g, x-y\rangle \geq 0$ for every $(y, g) \in G(T)$ implies $f \in T x$. Let $A: C \rightarrow H$ be a monotone, $k$-Lipschitzcontinuous mapping, and let $N_{C} v$ be the normal cone to $C$ at $v \in C$; that is,

$$
N_{C} v=\{w \in H:\langle v-u, w\rangle \geq 0, \forall u \in C\} .
$$

Define

$$
T v= \begin{cases}A v+N_{C} v, & \text { if } v \in C, \\ \emptyset, & \text { if } v \notin C .\end{cases}
$$

Then, $T$ is maximal monotone and $0 \in T v$ if and only if $v \in$ $\mathrm{VI}(C, A)$; see [39].

Lemma 15 (see [36, Lemma 2.6]). Let $C$ be a nonempty subset of a Hilbert space $H$ and $S: C \rightarrow C$ an asymptotically $k$-strict pseudocontractive mapping in the intermediate sense with sequence $\left\{\gamma_{n}\right\}$. Then

$$
\begin{aligned}
\left\|S^{n} x-S^{n} y\right\| \leq & \frac{1}{1-k} \\
& \quad \times(k\|x-y\| \\
& \left.\quad+\sqrt{\left(1+(1-k) \gamma_{n}\right)\|x-y\|^{2}+(1-k) c_{n}}\right)
\end{aligned}
$$

for all $x, y \in C$ and $n \geq 1$.

Lemma 16 (see [36, Lemma 2.7]). Let $C$ be a nonempty subset of a Hilbert space $H$ and $S: C \rightarrow C$ a uniformly continuous asymptotically $k$-strict pseudocontractive mapping in the intermediate sense with sequence $\left\{\gamma_{n}\right\}$. Let $\left\{x_{n}\right\}$ be a sequence in $C$ such that $\left\|x_{n}-x_{n+1}\right\| \rightarrow 0$ and $\left\|x_{n}-S^{n} x_{n}\right\| \rightarrow 0$ as $n \rightarrow \infty$. Then $\left\|x_{n}-S x_{n}\right\| \rightarrow 0$ as $n \rightarrow \infty$.

Lemma 17 (see Demiclosedness principle [36, Proposition 3.1]). Let $C$ be a nonempty closed convex subset of a Hilbert space $H$ and $S: C \rightarrow C$ a continuous asymptotically $k$-strict pseudocontractive mapping in the intermediate sense with sequence $\left\{\gamma_{n}\right\}$. Then $I-S$ is demiclosed at zero in the sense that if $\left\{x_{n}\right\}$ is a sequence in $C$ such that $x_{n} \rightarrow x \in C$ and $\lim \sup _{m \rightarrow \infty} \lim \sup _{n \rightarrow \infty}\left\|x_{n}-S^{m} x_{n}\right\|=0$, then $(I-S) x=0$. 
Lemma 18 (see [36, Proposition 3.2]). Let $C$ be a nonempty closed convex subset of a Hilbert space $H$ and $S: C \rightarrow C$ a continuous asymptotically $k$-strict pseudocontractive mapping in the intermediate sense with sequence $\left\{\gamma_{n}\right\}$ such that $\operatorname{Fix}(S) \neq \emptyset$. Then $\operatorname{Fix}(S)$ is closed and convex.

Remark 19. Lemmas 17 and 18 give some basic properties of an asymptotically $k$-strict pseudocontractive mapping in the intermediate sense with sequence $\left\{\gamma_{n}\right\}$. Moreover, Lemma 17 extends the Demiclosedness principles studied for certain classes of nonlinear mappings in Kim and Xu [35], Górnicki [40], $\mathrm{Xu}$ [41], and Marino and $\mathrm{Xu}$ [42].

Lemma 20 (see [43, page 80]). Let $\left\{a_{n}\right\}_{n=1}^{\infty},\left\{b_{n}\right\}_{n=1}^{\infty}$, and $\left\{\delta_{n}\right\}_{n=1}^{\infty}$ be sequences of nonnegative real numbers satisfying the inequality

$$
a_{n+1} \leq\left(1+\delta_{n}\right) a_{n}+b_{n}, \quad \forall n \geq 1 .
$$

If $\sum_{n=1}^{\infty} \delta_{n}<\infty$ and $\sum_{n=1}^{\infty} b_{n}<\infty$, then $\lim _{n \rightarrow \infty} a_{n}$ exists. If, in addition, $\left\{a_{n}\right\}_{n=1}^{\infty}$ has a subsequence which converges to zero, then $\lim _{n \rightarrow \infty} a_{n}=0$.

Recall that a Banach space $X$ is said to satisfy the Opial condition [38] if, for any given sequence $\left\{x_{n}\right\} \subset X$ which converges weakly to an element $x \in X$, there holds the inequality

$$
\limsup _{n \rightarrow \infty}\left\|x_{n}-x\right\|<\limsup _{n \rightarrow \infty}\left\|x_{n}-y\right\|, \quad \forall y \in X, \quad y \neq x .
$$

It is well known in [38] that every Hilbert space $H$ satisfies the Opial condition.

Lemma 21 (see [22, Proposition 3.1]). Let $C$ be a nonempty closed convex subset of a real Hilbert space $H$ and let $\left\{x_{n}\right\}$ be a sequence in $H$. Suppose that

$$
\left\|x_{n+1}-p\right\|^{2} \leq\left(1+\lambda_{n}\right)\left\|x_{n}-p\right\|^{2}+\delta_{n}, \quad \forall p \in C, n \geq 1,
$$

where $\left\{\lambda_{n}\right\}$ and $\left\{\delta_{n}\right\}$ are sequences of nonnegative real numbers such that $\sum_{n=1}^{\infty} \lambda_{n}<\infty$ and $\sum_{n=1}^{\infty} \delta_{n}<\infty$. Then $\left\{P_{C} x_{n}\right\}$ converges strongly in $C$.

Lemma 22 (see [44]). Let $C$ be a closed convex subset of a real Hilbert space $H$. Let $\left\{x_{n}\right\}$ be a sequence in $H$ and $u \in H$. Let $q=P_{C} u$. If $\left\{x_{n}\right\}$ is such that $\omega_{w}\left(x_{n}\right) \subset C$ and satisfies the condition

$$
\left\|x_{n}-u\right\| \leq\|u-q\|, \quad \forall n
$$

then $x_{n} \rightarrow$ qas $n \rightarrow \infty$.

\section{Strong Convergence Theorem}

In this section, we will introduce and analyze one iterative algorithm by using a composite shrinking projection method for finding a solution of the system of generalized equilibria with constraints of several problems: a generalized mixed equilibrium problem, finitely many variational inequalities, and the common fixed point problem of an asymptotically strict pseudocontractive mapping in the intermediate sense and infinitely many nonexpansive mappings in a real Hilbert space. Under appropriate conditions we will prove strong convergence of the proposed algorithm.

Theorem 23. Let $C$ be a nonempty closed convex subset of a real Hilbert space $H$. Let $N$ be an integer. Let $\Theta, \Theta_{1}, \Theta_{2}$ be three bifunctions from $\mathrm{C} \times \mathrm{C}$ to $\mathbf{R}$ satisfying (H1)-(H4) and let $\varphi: C \rightarrow \mathbf{R}$ be a lower semicontinuous and convex functional. Let $A, A_{k}: H \rightarrow H$ and $B_{i}: C \rightarrow H$ be $\zeta$ inverse strongly monotone, $\zeta_{k}$-inverse strongly monotone, and $\eta_{i}$-inverse strongly monotone, respectively, where $k \in\{1,2\}$ and $i \in\{1,2, \ldots, N\}$. Let $S: C \rightarrow C$ be a uniformly continuous asymptotically $k$-strict pseudocontractive mapping in the intermediate sense for some $0 \leq k<1$ with sequence $\left\{\gamma_{n}\right\} \subset[0, \infty)$ such that $\lim _{n \rightarrow \infty} \gamma_{n}=0$ and $\left\{c_{n}\right\} \subset[0, \infty)$ such that $\lim _{n \rightarrow \infty} c_{n}=0$. Let $\left\{T_{n}\right\}_{n=1}^{\infty}$ be a sequence of nonexpansive mappings on $H$ and $\left\{\lambda_{n}\right\}$ a sequence in $(0, b]$ for some $b \in$ $(0,1)$. Let $V$ be a $\bar{\gamma}$-strongly positive bounded linear operator with $\bar{\gamma} \in(1,2]$. Let $W_{n}$ be the $W$-mapping defined by (13). Assume that $\Omega:=\cap_{n=1}^{\infty} \operatorname{Fix}\left(T_{n}\right) \cap \operatorname{GMEP}(\Theta, \varphi, A) \cap \operatorname{SGEP}(G) \cap$ $\cap_{i=1}^{N} \operatorname{VI}\left(C, B_{i}\right) \cap \operatorname{Fix}(S)$ is nonempty and bounded where $G$ is defined as in Proposition CY. Let $\left\{r_{n}\right\}$ be a sequence in $[0,2 \zeta]$ and $\left\{\alpha_{n}\right\},\left\{\beta_{n}\right\},\left\{\sigma_{n}\right\}$, and $\left\{\delta_{n}\right\}$ sequences in $[0,1]$ such that $\lim _{n \rightarrow \infty} \sigma_{n}=0,0<\alpha \leq \alpha_{n} \leq 1$, and $k \leq \delta_{n} \leq d<1$. Pick any $x_{0} \in H$ and set $C_{1}=C, x_{1}=P_{C_{1}} x_{0}$. Let $\left\{x_{n}\right\}$ be a sequence generated by the following algorithm:

$$
\begin{gathered}
u_{n}=S_{r_{n}}^{(\Theta, \varphi)}\left(I-r_{n} A\right) x_{n}, \\
v_{n}=P_{C}\left(I-\lambda_{N, n} B_{N}\right) \\
\times P_{C}\left(I-\lambda_{N-1, n} B_{N-1}\right) \cdots P_{C}\left(I-\lambda_{1, n} B_{1}\right) u_{n}, \\
z_{n}=\beta_{n} x_{n}+\sigma_{n} G v_{n}+\left[\left(1-\beta_{n}\right) I-\sigma_{n} V\right] W_{n} G v_{n}, \\
k_{n}=\delta_{n} z_{n}+\left(1-\delta_{n}\right) S^{n} z_{n}, \\
y_{n}=\left(1-\alpha_{n}\right) x_{n}+\alpha_{n} k_{n}, \\
C_{n+1}=\left\{z \in C_{n}:\left\|y_{n}-z\right\|^{2} \leq\left\|x_{n}-z\right\|^{2}+\theta_{n}\right\}, \\
x_{n+1}=P_{C_{n+1}} x_{0}, \quad \forall n \geq 1,
\end{gathered}
$$

where $\theta_{n}=\left(\sigma_{n}+\gamma_{n}\right)\left(1+\gamma_{n}\right) \Delta_{n}+c_{n}, \Delta_{n}=\sup \left\{\left\|x_{n}-p\right\|^{2}+\right.$ $\left.\|(I-V) p\|^{2} /(\bar{\gamma}-1): p \in \Omega\right\}<\infty, v_{k} \in\left(0,2 \zeta_{k}\right), k \in\{1,2\}$, and $\left\{\lambda_{i, n}\right\} \subset\left[a_{i}, b_{i}\right] \subset\left(0,2 \eta_{i}\right), \forall i \in\{1,2, \ldots, N\}$. Assume that the following conditions are satisfied:

(i) $\mathrm{K}: H \rightarrow \mathbf{R}$ is strongly convex with constant $\sigma>0$ and its derivative $K^{\prime}$ is Lipschitz continuous with constant $v>0$ such that the function $x \mapsto\left\langle y-x, K^{\prime}(x)\right\rangle$ is weakly upper semicontinuous for each $y \in H$; 
(ii) for each $x \in H$, there exists a bounded subset $D_{x} \subset C$ and $z_{x} \in C$ such that, for any $y \notin D_{x}$,

$$
\begin{aligned}
& \Theta\left(y, z_{x}\right)+\varphi\left(z_{x}\right)-\varphi(y) \\
& \quad+\frac{1}{r}\left\langle K^{\prime}(y)-K^{\prime}(x), z_{x}-y\right\rangle<0 ;
\end{aligned}
$$

(iii) $0<\liminf _{n \rightarrow \infty} \beta_{n} \leq \limsup _{n \rightarrow \infty} \beta_{n}<1$ and $0<$ $\liminf _{n \rightarrow \infty} r_{n} \leq \lim \sup _{n \rightarrow \infty} r_{n}<2 \zeta$.

Then $\left\{x_{n}\right\}$ converges strongly to $x^{*}=P_{\Omega} x_{0}$ provided that $S_{r}^{(\Theta, \varphi)}$ is firmly nonexpansive.

Proof. As $\lim _{n \rightarrow \infty} \sigma_{n}=0,0<\liminf _{n \rightarrow \infty} \beta_{n} \leq$ $\lim \sup _{n \rightarrow \infty} \beta_{n}<1$ and $0<\liminf _{n \rightarrow \infty} r_{n} \leq$ $\lim \sup _{n \rightarrow \infty} r_{n}<2 \zeta$, we may assume, without loss of generality, that $\left\{\beta_{n}\right\} \subset[a, \widehat{a}] \subset[0,1],\left\{r_{n}\right\} \subset[c, \widehat{c}] \subset(0,2 \zeta)$ and $\beta_{n}+\sigma_{n}\|V\| \leq 1$ for all $n \geq 1$. Since $V$ is a $\bar{\gamma}$-strongly positive bounded linear operator on $H$, we know that

$$
\|V\|=\sup \{\langle V u, u\rangle: u \in H,\|u\|=1\} \geq \bar{\gamma}>1 .
$$

Taking into account that $\beta_{n}+\sigma_{n}\|V\| \leq 1$ for all $n \geq 1$, we have

$$
\begin{aligned}
\left\langle\left(\left(1-\beta_{n}\right) I-\sigma_{n} V\right) u, u\right\rangle & =1-\beta_{n}-\sigma_{n}\langle V u, u\rangle \\
& \geq 1-\beta_{n}-\sigma_{n}\|V\| \\
& \geq 0 ;
\end{aligned}
$$

that is, $\left(1-\beta_{n}\right) I-\sigma_{n} V$ is positive. It follows that

$$
\begin{aligned}
\|( & \left.-\beta_{n}\right) I-\sigma_{n} V \| \\
& =\sup \left\{\left\langle\left(\left(1-\beta_{n}\right) I-\sigma_{n} V\right) u, u\right\rangle: u \in H,\|u\|=1\right\} \\
& =\sup \left\{1-\beta_{n}-\sigma_{n}\langle V u, u\rangle: u \in H,\|u\|=1\right\} \\
& \leq 1-\beta_{n}-\sigma_{n} \bar{\gamma} .
\end{aligned}
$$

Put

$$
\Lambda_{n}^{i}=P_{C}\left(I-\lambda_{i, n} B_{i}\right) P_{C}\left(I-\lambda_{i-1, n} B_{i-1}\right) \cdots P_{C}\left(I-\lambda_{1, n} B_{1}\right)
$$

for all $i \in\{1,2, \ldots, N\}$, and $\Lambda_{n}^{0}=I$, where $I$ is the identity mapping on $H$. Then we have $v_{n}=\Lambda_{n}^{N} u_{n}$.

We divide the rest of the proof into several steps.

Step 1. We show that $\left\{x_{n}\right\}$ is well defined. It is obvious that $C_{n}$ is closed and convex. As the defining inequality in $C_{n}$ is equivalent to the inequality

$$
\left\langle 2\left(x_{n}-y_{n}\right), z\right\rangle \leq\left\|x_{n}\right\|^{2}-\left\|y_{n}\right\|^{2}+\theta_{n},
$$

by Lemma 14 we know that $C_{n}$ is convex for every $n \geq 1$.

First of all, let us show that $\Omega \subset C_{n}$ for all $n \geq 1$. Suppose that $\Omega \subset C_{n}$ for some $n \geq 1$. Take $p \in \Omega$ arbitrarily. Since
$p=S_{r_{n}}^{(\Theta, \varphi)}\left(p-r_{n} A p\right), A$ is $\zeta$-inverse strongly monotone, and $0 \leq r_{n} \leq 2 \zeta$, we have, for any $n \geq 1$,

$$
\begin{aligned}
\| u_{n}- & p \|^{2} \\
= & \left\|S_{r_{n}}^{(\Theta, \varphi)}\left(I-r_{n} A\right) x_{n}-S_{r_{n}}^{(\Theta, \varphi)}\left(I-r_{n} A\right) p\right\|^{2} \\
\leq & \left\|\left(I-r_{n} A\right) x_{n}-\left(I-r_{n} A\right) p\right\|^{2} \\
= & \left\|\left(x_{n}-p\right)-r_{n}\left(A x_{n}-A p\right)\right\|^{2} \\
= & \left\|x_{n}-p\right\|^{2}-2 r_{n}\left\langle x_{n}-p, A x_{n}-A p\right\rangle \\
& +r_{n}^{2}\left\|A x_{n}-A p\right\|^{2} \\
\leq & \left\|x_{n}-p\right\|^{2}-2 r_{n} \zeta\left\|A x_{n}-A p\right\|^{2} \\
& +r_{n}^{2}\left\|A x_{n}-A p\right\|^{2} \\
= & \left\|x_{n}-p\right\|^{2}+r_{n}\left(r_{n}-2 \zeta\right)\left\|A x_{n}-A p\right\|^{2} \\
\leq & \left\|x_{n}-p\right\|^{2} .
\end{aligned}
$$

Since $p=P_{C}\left(I-\lambda_{i, n} B_{i}\right) p, \Lambda_{n}^{i} p=p$, and $B_{i}$ is $\eta_{i}$-inverse strongly monotone, where $\lambda_{i, n} \in\left(0,2 \eta_{i}\right), i \in\{1,2, \ldots, N\}$, by Proposition 5(iii) we deduce that for each $n \geq 1$

$$
\begin{aligned}
& \left\|v_{n}-p\right\| \\
& \quad=\left\|P_{C}\left(I-\lambda_{N, n} B_{N}\right) \Lambda_{n}^{N-1} u_{n}-P_{C}\left(I-\lambda_{N, n} B_{N}\right) \Lambda_{n}^{N-1} p\right\| \\
& \quad \leq\left\|\left(I-\lambda_{N, n} B_{N}\right) \Lambda_{n}^{N-1} u_{n}-\left(I-\lambda_{N, n} B_{N}\right) \Lambda_{n}^{N-1} p\right\| \\
& \quad \leq\left\|\Lambda_{n}^{N-1} u_{n}-\Lambda_{n}^{N-1} p\right\| \\
& \quad \vdots \\
& \quad \leq\left\|\Lambda_{n}^{0} x_{n}-\Lambda_{n}^{0} p\right\| \\
& \quad=\left\|u_{n}-p\right\| .
\end{aligned}
$$

Combining (56) and (57), we have

$$
\left\|v_{n}-p\right\| \leq\left\|x_{n}-p\right\|
$$

Since $p=G p=T_{\nu_{1}}^{\Theta_{1}}\left(I-\nu_{1} A_{1}\right) T_{\nu_{2}}^{\Theta_{2}}\left(I-\nu_{2} A_{2}\right) p, A_{k}$ is $\zeta_{k}$-inverse strongly monotone, for $k=1,2$, and $0 \leq \nu_{k} \leq 2 \zeta_{k}$ for $k=1,2$, 
we deduce that, for any $n \geq 1$,

$$
\begin{aligned}
\left\|G v_{n}-p\right\|^{2} & \| T_{v_{1}}^{\Theta_{1}}\left(I-v_{1} A_{1}\right) T_{v_{2}}^{\Theta_{2}}\left(I-v_{2} A_{2}\right) v_{n} \\
& \quad-T_{v_{1}}^{\Theta_{1}}\left(I-v_{1} A_{1}\right) T_{v_{2}}^{\Theta_{2}}\left(I-v_{2} A_{2}\right) p \|^{2} \\
\leq & \|\left(I-v_{1} A_{1}\right) T_{v_{2}}^{\Theta_{2}}\left(I-v_{2} A_{2}\right) v_{n} \\
& \quad-\left(I-v_{1} A_{1}\right) T_{v_{2}}^{\Theta_{2}}\left(I-v_{2} A_{2}\right) p \|^{2} \\
= & \|\left[T_{v_{2}}^{\Theta_{2}}\left(I-v_{2} A_{2}\right) v_{n}-T_{v_{2}}^{\Theta_{2}}\left(I-v_{2} A_{2}\right) p\right] \\
& \quad-v_{1}\left[A_{1} T_{v_{2}}^{\Theta_{2}}\left(I-v_{2} A_{2}\right) v_{n}-A_{1} T_{v_{2}}^{\Theta_{2}}\left(I-v_{2} A_{2}\right) p\right] \|^{2} \\
\leq & \left\|T_{v_{2}}^{\Theta_{2}}\left(I-v_{2} A_{2}\right) v_{n}-T_{v_{2}}^{\Theta_{2}}\left(I-v_{2} A_{2}\right) p\right\|^{2} \\
& +v_{1}\left(v_{1}-2 \zeta_{1}\right) \\
& \times\left\|A_{1} T_{v_{2}}^{\Theta_{2}}\left(I-v_{2} A_{2}\right) v_{n}-A_{1} T_{v_{2}}^{\Theta_{2}}\left(I-v_{2} A_{2}\right) p\right\|^{2} \\
\leq & \left\|T_{v_{2}}^{\Theta_{2}}\left(I-v_{2} A_{2}\right) v_{n}-T_{v_{2}}^{\Theta_{2}}\left(I-v_{2} A_{2}\right) p\right\|^{2} \\
\leq & \left\|\left(I-v_{2} A_{2}\right) v_{n}-\left(I-v_{2} A_{2}\right) p\right\|^{2} \\
= & \left\|\left(v_{n}-p\right)-v_{2}\left(A_{2} v_{n}-A_{2} p\right)\right\|^{2} \\
\leq & \left\|v_{n}-p\right\|^{2}+v_{2}\left(v_{2}-2 \zeta_{2}\right)\left\|A_{2} v_{n}-A_{2} p\right\|^{2} \\
\leq & \left\|v_{n}-p\right\|^{2} .
\end{aligned}
$$

(This shows that $G$ is nonexpansive.) Also, from (49), (53), (58), and (59) it follows that

$$
\begin{aligned}
\| z_{n}- & p \| \\
= & \| \beta_{n}\left(x_{n}-p\right)+\sigma_{n}\left(G v_{n}-p\right)+\left[\left(1-\beta_{n}\right) I-\sigma_{n} V\right] \\
& \quad \times\left(W_{n} G v_{n}-p\right)+\sigma_{n}(I-V) p \| \\
\leq & \beta_{n}\left\|x_{n}-p\right\|+\sigma_{n}\left\|G v_{n}-p\right\| \\
& +\left\|\left[\left(1-\beta_{n}\right) I-\sigma_{n} V\right]\left(W_{n} G v_{n}-p\right)\right\| \\
& +\sigma_{n}\|(I-V) p\| \\
\leq & \beta_{n}\left\|x_{n}-p\right\|+\sigma_{n}\left\|G v_{n}-p\right\| \\
& +\left(1-\beta_{n}-\sigma_{n} \bar{\gamma}\right)\left\|W_{n} G v_{n}-p\right\| \\
& +\sigma_{n}\|(I-V) p\| \\
\leq & \beta_{n}\left\|x_{n}-p\right\|+\gamma_{n}\left\|G v_{n}-p\right\| \\
& +\left(1-\beta_{n}-\gamma_{n} \bar{\gamma}\right)\left\|G v_{n}-p\right\| \\
& +\gamma_{n}\|(I-V) p\| \\
= & \beta_{n}\left\|x_{n}-p\right\|+\left(1-\beta_{n}-\sigma_{n}(\bar{\gamma}-1)\right)
\end{aligned}
$$

$$
\begin{aligned}
& \times\left\|G v_{n}-p\right\|+\sigma_{n}\|(I-V) p\| \\
\leq & \beta_{n}\left\|x_{n}-p\right\|+\left(1-\beta_{n}-\sigma_{n}(\bar{\gamma}-1)\right) \\
& \times\left\|v_{n}-p\right\|+\sigma_{n}\|(I-V) p\| \\
\leq & \beta_{n}\left\|x_{n}-p\right\|+\left(1-\beta_{n}-\sigma_{n}(\bar{\gamma}-1)\right) \\
& \times\left\|x_{n}-p\right\|+\sigma_{n}\|(I-V) p\| \\
= & \left(1-\sigma_{n}(\bar{\gamma}-1)\right)\left\|x_{n}-p\right\|+\sigma_{n}\|(I-V) p\| \\
= & \left(1-\sigma_{n}(\bar{\gamma}-1)\right)\left\|x_{n}-p\right\|+\sigma_{n}(\bar{\gamma}-1) \frac{\|(I-V) p\|}{\bar{\gamma}-1},
\end{aligned}
$$

which hence yields

$$
\begin{aligned}
\left\|z_{n}-p\right\|^{2} \leq & \left(1-\sigma_{n}(\bar{\gamma}-1)\right)\left\|x_{n}-p\right\|^{2} \\
& +\sigma_{n}(\bar{\gamma}-1) \frac{\|(I-V) p\|^{2}}{(\bar{\gamma}-1)^{2}} \\
\leq & \left\|x_{n}-p\right\|^{2}+\sigma_{n} \frac{\|(I-V) p\|^{2}}{\bar{\gamma}-1} .
\end{aligned}
$$

By Lemma 9(b), we deduce from (49) and (61) that

$$
\begin{aligned}
\| k_{n}- & p \|^{2} \\
= & \left\|\delta_{n}\left(z_{n}-p\right)+\left(1-\delta_{n}\right)\left(S^{n} z_{n}-p\right)\right\|^{2} \\
= & \delta_{n}\left\|z_{n}-p\right\|^{2}+\left(1-\delta_{n}\right)\left\|S^{n} z_{n}-p\right\|^{2} \\
& -\delta_{n}\left(1-\delta_{n}\right)\left\|z_{n}-S^{n} z_{n}\right\|^{2} \\
\leq & \delta_{n}\left\|z_{n}-p\right\|^{2}+\left(1-\delta_{n}\right) \\
& \times\left[\left(1+\gamma_{n}\right)\left\|z_{n}-p\right\|^{2}+k\left\|z_{n}-S^{n} z_{n}\right\|^{2}+c_{n}\right] \\
& -\delta_{n}\left(1-\delta_{n}\right)\left\|z_{n}-S^{n} z_{n}\right\|^{2} \\
= & {\left[1+\gamma_{n}\left(1-\delta_{n}\right)\right]\left\|z_{n}-p\right\|^{2}+\left(1-\delta_{n}\right) } \\
& \times\left(k-\delta_{n}\right)\left\|z_{n}-S^{n} z_{n}\right\|^{2}+\left(1-\delta_{n}\right) c_{n} \\
\leq & \left(1+\gamma_{n}\right)\left\|z_{n}-p\right\|^{2}+\left(1-\delta_{n}\right) \\
& \times\left(k-\delta_{n}\right)\left\|z_{n}-S^{n} z_{n}\right\|^{2}+c_{n} \\
\leq & \left(1+\gamma_{n}\right)\left\|z_{n}-p\right\|^{2}+c_{n} \\
\leq & \left(1+\gamma_{n}\right)\left(\left\|x_{n}-p\right\|^{2}+\sigma_{n} \frac{\|(I-V) p\|^{2}}{\bar{\gamma}-1}\right)+c_{n} .
\end{aligned}
$$


So, from (49) and (62) we get

$$
\begin{aligned}
& \left\|y_{n}-p\right\|^{2} \\
& =\left\|\left(1-\alpha_{n}\right)\left(x_{n}-p\right)+\alpha_{n}\left(k_{n}-p\right)\right\|^{2} \\
& \leq\left(1-\alpha_{n}\right)\left\|x_{n}-p\right\|^{2}+\alpha_{n}\left\|k_{n}-p\right\|^{2} \\
& \leq\left(1-\alpha_{n}\right)\left\|x_{n}-p\right\|^{2} \\
& +\alpha_{n}\left[\left(1+\gamma_{n}\right)\left(\left\|x_{n}-p\right\|^{2}+\sigma_{n} \frac{\|(I-V) p\|^{2}}{\bar{\gamma}-1}\right)+c_{n}\right] \\
& \leq\left(1+\gamma_{n}\right)\left(\left\|x_{n}-p\right\|^{2}+\sigma_{n} \frac{\|(I-V) p\|^{2}}{\bar{\gamma}-1}\right)+c_{n} \\
& =\left\|x_{n}-p\right\|^{2}+\gamma_{n}\left\|x_{n}-p\right\|^{2} \\
& +\left(1+\gamma_{n}\right) \sigma_{n} \frac{\|(I-V) p\|^{2}}{\bar{\gamma}-1}+c_{n} \\
& \leq\left\|x_{n}-p\right\|^{2}+\gamma_{n}\left(1+\gamma_{n}\right)\left\|x_{n}-p\right\|^{2} \\
& +\sigma_{n}\left(1+\gamma_{n}\right) \frac{\|(I-V) p\|^{2}}{\bar{\gamma}-1}+c_{n} \\
& \leq\left\|x_{n}-p\right\|^{2}+\left(\gamma_{n}+\sigma_{n}\right)\left(1+\gamma_{n}\right)\left\|x_{n}-p\right\|^{2} \\
& +\left(\sigma_{n}+\gamma_{n}\right)\left(1+\gamma_{n}\right) \frac{\|(I-V) p\|^{2}}{\bar{\gamma}-1}+c_{n} \\
& =\left\|x_{n}-p\right\|^{2}+\left(\sigma_{n}+\gamma_{n}\right)\left(1+\gamma_{n}\right) \\
& \times\left(\left\|x_{n}-p\right\|^{2}+\frac{\|(I-V) p\|^{2}}{\bar{\gamma}-1}\right)+c_{n} \\
& \leq\left\|x_{n}-p\right\|^{2}+\left(\sigma_{n}+\gamma_{n}\right)\left(1+\gamma_{n}\right) \Delta_{n}+c_{n} \\
& =\left\|x_{n}-p\right\|^{2}+\theta_{n} \text {, }
\end{aligned}
$$

where $\theta_{n}=\left(\sigma_{n}+\gamma_{n}\right)\left(1+\gamma_{n}\right) \Delta_{n}+c_{n}$ and $\Delta_{n}=\sup \left\{\left\|x_{n}-p\right\|^{2}+\right.$ $\left.\|(I-V) p\|^{2} /(\bar{\gamma}-1): p \in \Omega\right\}<\infty$. Hence $p \in C_{n+1}$. This implies that $\Omega \subset C_{n}$ for all $n \geq 1$. Therefore, $\left\{x_{n}\right\}$ is well defined.

Step 2. We prove that $\left\|x_{n}-k_{n}\right\| \rightarrow 0,\left\|x_{n}-z_{n}\right\| \rightarrow 0$, and $\left\|S^{n} z_{n}-z_{n}\right\| \rightarrow 0$ as $n \rightarrow \infty$.

Indeed, let $x^{*}=P_{\Omega} x_{0}$. From $x_{n}=P_{C_{n}} x_{0}$ and $x^{*} \in \Omega \subset$ $C_{n}$, we obtain

$$
\left\|x_{n}-x_{0}\right\| \leq\left\|x^{*}-x_{0}\right\| .
$$

This implies that $\left\{x_{n}\right\}$ is bounded and hence $\left\{u_{n}\right\},\left\{v_{n}\right\},\left\{z_{n}\right\}$, $\left\{k_{n}\right\}$, and $\left\{y_{n}\right\}$ are also bounded. Since $x_{n+1} \in C_{n+1} \subset C_{n}$ and $x_{n}=P_{C_{n}} x_{0}$, we have

$$
\left\|x_{n}-x_{0}\right\| \leq\left\|x_{n+1}-x_{0}\right\|, \quad \forall n \geq 1 .
$$

Therefore $\lim _{n \rightarrow \infty}\left\|x_{n}-x_{0}\right\|$ exists. From $x_{n}=P_{C_{n}} x_{0}, x_{n+1} \epsilon$ $C_{n+1} \subset C_{n}$, by Proposition 5(ii) we obtain

$$
\left\|x_{n+1}-x_{n}\right\|^{2} \leq\left\|x_{0}-x_{n+1}\right\|^{2}-\left\|x_{0}-x_{n}\right\|^{2},
$$

which implies

$$
\lim _{n \rightarrow \infty}\left\|x_{n+1}-x_{n}\right\|=0
$$

It follows from $x_{n+1} \in C_{n+1}$ that $\left\|y_{n}-x_{n+1}\right\|^{2} \leq\left\|x_{n}-x_{n+1}\right\|^{2}+$ $\theta_{n}$ and hence

$$
\begin{aligned}
\| x_{n} & -y_{n} \|^{2} \\
& \leq 2\left(\left\|x_{n}-x_{n+1}\right\|^{2}+\left\|x_{n+1}-y_{n}\right\|^{2}\right) \\
& \leq 2\left(\left\|x_{n}-x_{n+1}\right\|^{2}+\left\|x_{n}-x_{n+1}\right\|^{2}+\theta_{n}\right) \\
& =2\left(2\left\|x_{n}-x_{n+1}\right\|^{2}+\theta_{n}\right) .
\end{aligned}
$$

From (67) and $\lim _{n \rightarrow \infty} \theta_{n}=0$, we have

$$
\lim _{n \rightarrow \infty}\left\|x_{n}-y_{n}\right\|=0 \text {. }
$$

Since $y_{n}-x_{n}=\alpha_{n}\left(k_{n}-x_{n}\right)$ and $0<\alpha \leq \alpha_{n} \leq 1$, we have

$$
\alpha\left\|k_{n}-x_{n}\right\| \leq \alpha_{n}\left\|k_{n}-x_{n}\right\|=\left\|y_{n}-x_{n}\right\|,
$$

which immediately leads to

$$
\lim _{n \rightarrow \infty}\left\|k_{n}-x_{n}\right\|=0 \text {. }
$$

Also, utilizing Lemmas 8 and 9(b) we obtain from (49), (58), (59), and (62) that

$$
\begin{aligned}
\| z_{n}- & p \|^{2} \\
= & \left\|\beta_{n} x_{n}+\sigma_{n} G v_{n}+\left[\left(1-\beta_{n}\right) I-\sigma_{n} V\right] W_{n} G v_{n}-p\right\|^{2} \\
= & \| \beta_{n}\left(x_{n}-p\right)+\left(1-\beta_{n}\right)\left(W_{n} G v_{n}-p\right) \\
& +\sigma_{n}\left(G v_{n}-V W_{n} G v_{n}\right) \|^{2} \\
\leq & \left\|\beta_{n}\left(x_{n}-p\right)+\left(1-\beta_{n}\right)\left(W_{n} G v_{n}-p\right)\right\|^{2} \\
& +2 \sigma_{n}\left\langle\left(G v_{n}-V W_{n} G v_{n}\right), z_{n}-p\right\rangle \\
= & \beta_{n}\left\|x_{n}-p\right\|^{2}+\left(1-\beta_{n}\right)\left\|W_{n} G v_{n}-p\right\|^{2} \\
& -\beta_{n}\left(1-\beta_{n}\right)\left\|x_{n}-W_{n} G v_{n}\right\|^{2} \\
& +2 \sigma_{n}\left\langle\left(G v_{n}-V W_{n} G v_{n}\right), z_{n}-p\right\rangle \\
\leq & \beta_{n}\left\|x_{n}-p\right\|^{2}+\left(1-\beta_{n}\right)\left\|G v_{n}-p\right\|^{2} \\
& -\beta_{n}\left(1-\beta_{n}\right)\left\|x_{n}-W_{n} G v_{n}\right\|^{2} \\
& +2 \sigma_{n}\left\|G v_{n}-V W_{n} G v_{n}\right\|\left\|z_{n}-p\right\| \\
\leq & \beta_{n}\left\|x_{n}-p\right\|^{2}+\left(1-\beta_{n}\right)\left\|v_{n}-p\right\|^{2} \\
& -\beta_{n}\left(1-\beta_{n}\right)\left\|x_{n}-W_{n} G v_{n}\right\|^{2} \\
& +2 \sigma_{n}\left\|G v_{n}-V W_{n} G v_{n}\right\|\left\|z_{n}-p\right\|
\end{aligned}
$$




$$
\begin{aligned}
\leq & \beta_{n}\left\|x_{n}-p\right\|^{2}+\left(1-\beta_{n}\right)\left\|x_{n}-p\right\|^{2} \\
& -\beta_{n}\left(1-\beta_{n}\right)\left\|x_{n}-W_{n} G v_{n}\right\|^{2} \\
& +2 \sigma_{n}\left\|G v_{n}-V W_{n} G v_{n}\right\|\left\|z_{n}-p\right\| \\
= & \left\|x_{n}-p\right\|^{2}-\beta_{n}\left(1-\beta_{n}\right)\left\|x_{n}-W_{n} G v_{n}\right\|^{2} \\
& +2 \sigma_{n}\left\|G v_{n}-V W_{n} G v_{n}\right\|\left\|z_{n}-p\right\|,
\end{aligned}
$$

and hence

$$
\begin{aligned}
& \left\|y_{n}-p\right\|^{2} \\
& \leq\left(1-\alpha_{n}\right)\left\|x_{n}-p\right\|^{2}+\alpha_{n}\left\|k_{n}-p\right\|^{2} \\
& \leq\left(1-\alpha_{n}\right)\left\|x_{n}-p\right\|^{2} \\
& +\alpha_{n}\left[\left(1+\gamma_{n}\right)\left\|z_{n}-p\right\|^{2}+c_{n}\right] \\
& \leq\left(1-\alpha_{n}\right)\left\|x_{n}-p\right\|^{2} \\
& +\alpha_{n}\left[\left(1+\gamma_{n}\right)\right. \\
& \times\left(\left\|x_{n}-p\right\|^{2}-\beta_{n}\left(1-\beta_{n}\right)\right. \\
& \times\left\|x_{n}-W_{n} G v_{n}\right\|^{2} \\
& \left.\left.+2 \sigma_{n}\left\|G v_{n}-V W_{n} G v_{n}\right\|\left\|z_{n}-p\right\|\right)+c_{n}\right] \\
& \leq\left(1-\alpha_{n}\right)\left\|x_{n}-p\right\|^{2}+\alpha_{n}\left(1+\gamma_{n}\right) \\
& \times\left(\left\|x_{n}-p\right\|^{2}-\beta_{n}\left(1-\beta_{n}\right)\left\|x_{n}-W_{n} G v_{n}\right\|^{2}\right. \\
& \left.+2 \sigma_{n}\left\|G v_{n}-V W_{n} G v_{n}\right\|\left\|z_{n}-p\right\|\right)+c_{n} \\
& \leq\left(1-\alpha_{n}\right)\left\|x_{n}-p\right\|^{2}+\alpha_{n}\left(1+\gamma_{n}\right)\left\|x_{n}-p\right\|^{2} \\
& -\alpha_{n}\left(1+\gamma_{n}\right) \beta_{n}\left(1-\beta_{n}\right)\left\|x_{n}-W_{n} G v_{n}\right\|^{2} \\
& +\left(1+\gamma_{n}\right) 2 \sigma_{n}\left\|G v_{n}-V W_{n} G v_{n}\right\|\left\|z_{n}-p\right\|+c_{n} \\
& \leq\left(1+\gamma_{n}\right)\left\|x_{n}-p\right\|^{2}-\alpha_{n}\left(1+\gamma_{n}\right) \\
& \times \beta_{n}\left(1-\beta_{n}\right)\left\|x_{n}-W_{n} G v_{n}\right\|^{2} \\
& +2 \sigma_{n}\left(1+\gamma_{n}\right)\left\|G v_{n}-V W_{n} G v_{n}\right\|\left\|z_{n}-p\right\|+c_{n} .
\end{aligned}
$$

So, it follows that

$$
\begin{aligned}
\alpha(1 & \left.+\gamma_{n}\right) a(1-\widehat{a})\left\|x_{n}-W_{n} G v_{n}\right\|^{2} \\
\leq & \alpha_{n}\left(1+\gamma_{n}\right) \beta_{n}\left(1-\beta_{n}\right)\left\|x_{n}-W_{n} G v_{n}\right\|^{2} \\
\leq & \left\|x_{n}-p\right\|^{2}-\left\|y_{n}-p\right\|^{2}+\gamma_{n}\left\|x_{n}-p\right\|^{2} \\
& +2 \sigma_{n}\left(1+\gamma_{n}\right)\left\|G v_{n}-V W_{n} G v_{n}\right\|\left\|z_{n}-p\right\|+c_{n}
\end{aligned}
$$

$$
\begin{aligned}
\leq & \left\|x_{n}-y_{n}\right\|\left(\left\|x_{n}-p\right\|+\left\|y_{n}-p\right\|\right)+\gamma_{n}\left\|x_{n}-p\right\|^{2} \\
& +2 \sigma_{n}\left(1+\gamma_{n}\right)\left\|G v_{n}-V W_{n} G v_{n}\right\|\left\|z_{n}-p\right\|+c_{n} .
\end{aligned}
$$

Since $\lim _{n \rightarrow \infty} \sigma_{n}=0, \lim _{n \rightarrow \infty} \gamma_{n}=0$, and $\lim _{n \rightarrow \infty} c_{n}=0$, it follows from (69) and the boundedness of $\left\{x_{n}\right\},\left\{y_{n}\right\},\left\{z_{n}\right\}$, and $\left\{v_{n}\right\}$ that

$$
\lim _{n \rightarrow \infty}\left\|x_{n}-W_{n} G v_{n}\right\|=0
$$

Note that

$$
\begin{aligned}
& \left\|z_{n}-x_{n}\right\| \\
& \quad=\left\|\left(1-\beta_{n}\right)\left(W_{n} G v_{n}-x_{n}\right)+\sigma_{n}\left(G v_{n}-V W_{n} G v_{n}\right)\right\| \\
& \quad \leq\left(1-\beta_{n}\right)\left\|W_{n} G v_{n}-x_{n}\right\|+\sigma_{n}\left\|G v_{n}-V W_{n} G v_{n}\right\| \\
& \quad \leq\left\|W_{n} G v_{n}-x_{n}\right\|+\sigma_{n}\left\|G v_{n}-V W_{n} G v_{n}\right\| .
\end{aligned}
$$

Hence, it follows from (75) and $\lim _{n \rightarrow \infty} \sigma_{n}=0$ that

$$
\lim _{n \rightarrow \infty}\left\|x_{n}-z_{n}\right\|=0
$$

Note that

$$
\left\|k_{n}-z_{n}\right\| \leq\left\|k_{n}-x_{n}\right\|+\left\|x_{n}-z_{n}\right\|
$$

Thus, we deduce from (71) and (77) that

$$
\lim _{n \rightarrow \infty}\left\|k_{n}-z_{n}\right\|=0
$$

Since $k_{n}-z_{n}=\left(1-\delta_{n}\right)\left(S^{n} z_{n}-z_{n}\right)$ and $k \leq \delta_{n} \leq d<1$, we have

$$
(1-d)\left\|S^{n} z_{n}-z_{n}\right\| \leq\left(1-\delta_{n}\right)\left\|S^{n} z_{n}-z_{n}\right\|=\left\|k_{n}-z_{n}\right\|,
$$

which, together with (79), yields

$$
\lim _{n \rightarrow \infty}\left\|S^{n} z_{n}-z_{n}\right\|=0
$$

Step 3. We prove that $\left\|x_{n}-u_{n}\right\| \rightarrow 0,\left\|x_{n}-v_{n}\right\| \rightarrow 0, \| v_{n}-$ $G v_{n}\|\rightarrow 0,\| x_{n}-W x_{n} \| \rightarrow 0$, and $\left\|z_{n}-S z_{n}\right\| \rightarrow 0$ as $n \rightarrow \infty$. Indeed, from (57), (59), and $\bar{\gamma} \in(1,2]$ it follows that

$$
\begin{aligned}
& \left\|z_{n}-p\right\|^{2} \\
& =\| \beta_{n}\left(x_{n}-p\right)+\sigma_{n}\left(G v_{n}-p\right) \\
& \quad+\left[\left(1-\beta_{n}\right) I-\sigma_{n} V\right]\left(W_{n} G v_{n}-p\right) \\
& \quad+\sigma_{n}(I-V) p \|^{2}
\end{aligned}
$$




$$
\begin{aligned}
\leq & \| \beta_{n}\left(x_{n}-p\right)+\sigma_{n}\left(G v_{n}-p\right) \\
& +\left[\left(1-\beta_{n}\right) I-\sigma_{n} V\right]\left(W_{n} G v_{n}-p\right) \|^{2} \\
& +2 \sigma_{n}\left\langle(I-V) p, z_{n}-p\right\rangle \\
\leq & {\left[\beta_{n}\left\|x_{n}-p\right\|+\sigma_{n}\left\|G v_{n}-p\right\|\right.} \\
& \left.+\left(1-\beta_{n}-\sigma_{n} \bar{\gamma}\right)\left\|W_{n} G v_{n}-p\right\|\right]^{2} \\
& +2 \sigma_{n}\|(I-V) p\|\left\|z_{n}-p\right\| \\
\leq & {\left[\beta_{n}\left\|x_{n}-p\right\|+\sigma_{n}\left\|G v_{n}-p\right\|\right.} \\
& \left.+\left(1-\beta_{n}-\sigma_{n} \bar{\gamma}\right)\left\|G v_{n}-p\right\|\right]^{2} \\
& +2 \sigma_{n}\|(I-V) p\|\left\|z_{n}-p\right\| \\
= & {\left[\beta_{n}\left\|x_{n}-p\right\|+\left(1-\beta_{n}-\sigma_{n}(\bar{\gamma}-1)\right)\right.} \\
& \left.\times\left\|G v_{n}-p\right\|\right]^{2} \\
& +2 \sigma_{n}\|(I-V) p\|\left\|z_{n}-p\right\| \\
\leq & {\left[\beta_{n}\left\|x_{n}-p\right\|+\left(1-\beta_{n}\right)\left\|G v_{n}-p\right\|\right]^{2} } \\
+ & 2 \sigma_{n}\|(I-V) p\|\left\|z_{n}-p\right\| \\
\leq & \beta_{n}\left\|x_{n}-p\right\|^{2}+\left(1-\beta_{n}\right)\left\|G v_{n}-p\right\|^{2} \\
+ & 2 \sigma_{n}\|(I-V) p\|\left\|z_{n}-p\right\| \\
\leq & \beta_{n}\left\|x_{n}-p\right\|^{2}+\left(1-\beta_{n}\right)\left\|v_{n}-p\right\|^{2} \\
+ & 2 \sigma_{n}\|(I-V) p\|\left\|z_{n}-p\right\| \\
\leq & \beta_{n}\left\|x_{n}-p\right\|^{2}+\left(1-\beta_{n}\right)\left\|u_{n}-p\right\|^{2} \\
+ & 2 \sigma_{n}\|(I-V) p\|\left\|z_{n}-p\right\| . \\
&
\end{aligned}
$$

Next let us show that

$$
\lim _{n \rightarrow \infty}\left\|x_{n}-u_{n}\right\|=0 \text {. }
$$

For $p \in \Omega$, we find that

$$
\begin{aligned}
\| u_{n} & -p \|^{2} \\
& =\left\|S_{r_{n}}^{(\Theta, \varphi)}\left(I-r_{n} A\right) x_{n}-S_{r_{n}}^{(\Theta, \varphi)}\left(I-r_{n} A\right) p\right\|^{2} \\
& \leq\left\|\left(I-r_{n} A\right) x_{n}-\left(I-r_{n} A\right) p\right\|^{2} \\
& =\left\|x_{n}-p-r_{n}\left(A x_{n}-A p\right)\right\|^{2} \\
& \leq\left\|x_{n}-p\right\|^{2}+r_{n}\left(r_{n}-2 \zeta\right)\left\|A x_{n}-A p\right\|^{2} .
\end{aligned}
$$

Combining (82) and (84), we obtain

$$
\begin{aligned}
& \left\|z_{n}-p\right\|^{2} \\
& \leq \beta_{n}\left\|x_{n}-p\right\|^{2}+\left(1-\beta_{n}\right)\left\|u_{n}-p\right\|^{2} \\
& \quad+2 \sigma_{n}\|(I-V) p\|\left\|z_{n}-p\right\|
\end{aligned}
$$

$$
\begin{aligned}
\leq & \beta_{n}\left\|x_{n}-p\right\|^{2}+\left(1-\beta_{n}\right) \\
& \times\left[\left\|x_{n}-p\right\|^{2}+r_{n}\left(r_{n}-2 \zeta\right)\left\|A x_{n}-A p\right\|^{2}\right] \\
& +2 \sigma_{n}\|(I-V) p\|\left\|z_{n}-p\right\| \\
= & \left\|x_{n}-p\right\|^{2}+\left(1-\beta_{n}\right) r_{n}\left(r_{n}-2 \zeta\right) \\
& \times\left\|A x_{n}-A p\right\|^{2}+2 \sigma_{n}\|(I-V) p\|\left\|z_{n}-p\right\|,
\end{aligned}
$$

which immediately implies that

$$
\begin{aligned}
&(1-\widehat{a}) c(2 \zeta-\widehat{c})\left\|A x_{n}-A p\right\|^{2} \\
& \leq\left(1-\beta_{n}\right) r_{n}\left(2 \zeta-r_{n}\right)\left\|A x_{n}-A p\right\|^{2} \\
& \leq\left\|x_{n}-p\right\|^{2}-\left\|z_{n}-p\right\|^{2} \\
&+2 \sigma_{n}\|(I-V) p\|\left\|z_{n}-p\right\| \\
& \leq\left\|x_{n}-z_{n}\right\|\left(\left\|x_{n}-p\right\|+\left\|z_{n}-p\right\|\right) \\
&+2 \sigma_{n}\|(I-V) p\|\left\|z_{n}-p\right\| .
\end{aligned}
$$

Since $\lim _{n \rightarrow \infty} \sigma_{n}=0$ and $\left\{x_{n}\right\}$ and $\left\{z_{n}\right\}$ are bounded sequences, it follows from (77) that

$$
\lim _{n \rightarrow \infty}\left\|A x_{n}-A p\right\|=0
$$

Furthermore, from the firm nonexpansivity of $S_{r_{n}}^{(\Theta, \varphi)}$, we have

$$
\begin{aligned}
& \left\|u_{n}-p\right\|^{2} \\
& =\left\|S_{r_{n}}^{(\Theta, \varphi)}\left(I-r_{n} A\right) x_{n}-S_{r_{n}}^{(\Theta, \varphi)}\left(I-r_{n} A\right) p\right\|^{2} \\
& \leq\left\langle\left(I-r_{n} A\right) x_{n}-\left(I-r_{n} A\right) p, u_{n}-p\right\rangle \\
& =\frac{1}{2}\left[\left\|\left(I-r_{n} A\right) x_{n}-\left(I-r_{n} A\right) p\right\|^{2}+\left\|u_{n}-p\right\|^{2}\right. \\
& \left.\quad-\left\|\left(I-r_{n} A\right) x_{n}-\left(I-r_{n} A\right) p-\left(u_{n}-p\right)\right\|^{2}\right] \\
& \leq \frac{1}{2}\left[\left\|x_{n}-p\right\|^{2}+\left\|u_{n}-p\right\|^{2}\right. \\
& \left.\quad-\left\|x_{n}-u_{n}-r_{n}\left(A x_{n}-A p\right)\right\|^{2}\right] \\
& =\frac{1}{2}\left[\left\|x_{n}-p\right\|^{2}+\left\|u_{n}-p\right\|^{2}-\left\|x_{n}-u_{n}\right\|^{2}\right. \\
& \left.\quad+2 r_{n}\left\langle A x_{n}-A p, x_{n}-u_{n}\right\rangle-r_{n}^{2}\left\|A x_{n}-A p\right\|^{2}\right],
\end{aligned}
$$

which leads to

$$
\begin{aligned}
\left\|u_{n}-p\right\|^{2} \leq & \left\|x_{n}-p\right\|^{2}-\left\|x_{n}-u_{n}\right\|^{2} \\
& +2 r_{n}\left\|A x_{n}-A p\right\|\left\|x_{n}-u_{n}\right\| .
\end{aligned}
$$


From (82) and (89), we have

$$
\begin{aligned}
\| z_{n}- & p \|^{2} \\
\leq & \beta_{n}\left\|x_{n}-p\right\|^{2}+\left(1-\beta_{n}\right)\left\|u_{n}-p\right\|^{2} \\
& +2 \sigma_{n}\|(I-V) p\|\left\|z_{n}-p\right\| \\
\leq & \beta_{n}\left\|x_{n}-p\right\|^{2}+\left(1-\beta_{n}\right) \\
& \times\left[\left\|x_{n}-p\right\|^{2}-\left\|x_{n}-u_{n}\right\|^{2}\right. \\
& \left.+2 r_{n}\left\|A x_{n}-A p\right\|\left\|x_{n}-u_{n}\right\|\right] \\
& +2 \sigma_{n}\|(I-V) p\|\left\|z_{n}-p\right\| \\
\leq & \left\|x_{n}-p\right\|^{2}-\left(1-\beta_{n}\right)\left\|x_{n}-u_{n}\right\|^{2} \\
& +2 r_{n}\left\|A x_{n}-A p\right\|\left\|x_{n}-u_{n}\right\| \\
& +2 \sigma_{n}\|(I-V) p\|\left\|z_{n}-p\right\|,
\end{aligned}
$$

which hence implies that

$$
\begin{aligned}
\| x_{n}- & u_{n} \|^{2} \\
\leq & \left\|x_{n}-p\right\|^{2}-\left\|z_{n}-p\right\|^{2}+2 r_{n}\left\|A x_{n}-A p\right\| \\
& \times\left\|x_{n}-u_{n}\right\|+2 \sigma_{n}\|(I-V) p\|\left\|z_{n}-p\right\| \\
\leq & \left\|x_{n}-z_{n}\right\|\left(\left\|x_{n}-p\right\|+\left\|z_{n}-p\right\|\right) \\
& +2 r_{n}\left\|A x_{n}-A p\right\|\left\|x_{n}-u_{n}\right\| \\
& +2 \sigma_{n}\|(I-V) p\|\left\|z_{n}-p\right\| .
\end{aligned}
$$

Since $\lim _{n \rightarrow \infty} \sigma_{n}=0$ and $\left\{x_{n}\right\},\left\{u_{n}\right\}$, and $\left\{z_{n}\right\}$ are bounded sequences, it follows from (77) and (87) that (83) holds.

Next we show that $\lim _{n \rightarrow \infty}\left\|B_{i} \Lambda_{n}^{i} u_{n}-B_{i} p\right\|=0, i=$ $1,2, \ldots, N$. As a matter of fact, observe that

$$
\begin{aligned}
& \left\|\Lambda_{n}^{i} u_{n}-p\right\|^{2} \\
& \quad=\left\|P_{C}\left(I-\lambda_{i, n} B_{i}\right) \Lambda_{n}^{i-1} u_{n}-P_{C}\left(I-\lambda_{i, n} B_{i}\right) p\right\|^{2} \\
& \quad \leq\left\|\left(I-\lambda_{i, n} B_{i}\right) \Lambda_{n}^{i-1} u_{n}-\left(I-\lambda_{i, n} B_{i}\right) p\right\|^{2} \\
& \quad \leq\left\|\Lambda_{n}^{i-1} u_{n}-p\right\|^{2}+\lambda_{i, n}\left(\lambda_{i, n}-2 \eta_{i}\right)\left\|B_{i} \Lambda_{n}^{i-1} u_{n}-B_{i} p\right\|^{2} \\
& \quad \leq\left\|u_{n}-p\right\|^{2}+\lambda_{i, n}\left(\lambda_{i, n}-2 \eta_{i}\right)\left\|B_{i} \Lambda_{n}^{i-1} u_{n}-B_{i} p\right\|^{2} \\
& \quad \leq\left\|x_{n}-p\right\|^{2}+\lambda_{i, n}\left(\lambda_{i, n}-2 \eta_{i}\right)\left\|B_{i} \Lambda_{n}^{i-1} u_{n}-B_{i} p\right\|^{2} .
\end{aligned}
$$

Combining (59), (82), and (92), we have

$$
\begin{aligned}
\left\|z_{n}-p\right\|^{2} & \\
\leq & \beta_{n}\left\|x_{n}-p\right\|^{2}+\left(1-\beta_{n}\right)\left\|v_{n}-p\right\|^{2} \\
& +2 \sigma_{n}\|(I-V) p\|\left\|z_{n}-p\right\|
\end{aligned}
$$

$$
\begin{aligned}
\leq & \beta_{n}\left\|x_{n}-p\right\|^{2}+\left(1-\beta_{n}\right)\left\|\Lambda_{n}^{i} u_{n}-p\right\|^{2} \\
& +2 \sigma_{n}\|(I-V) p\|\left\|z_{n}-p\right\| \\
\leq & \beta_{n}\left\|x_{n}-p\right\|^{2}+\left(1-\beta_{n}\right) \\
& \times\left[\left\|x_{n}-p\right\|^{2}+\lambda_{i, n}\left(\lambda_{i, n}-2 \eta_{i}\right)\right. \\
& \left.\times\left\|B_{i} \Lambda_{n}^{i-1} u_{n}-B_{i} p\right\|^{2}\right] \\
& +2 \sigma_{n}\|(I-V) p\|\left\|z_{n}-p\right\| \\
= & \left\|x_{n}-p\right\|^{2}+\left(1-\beta_{n}\right) \lambda_{i, n} \\
& \times\left(\lambda_{i, n}-2 \eta_{i}\right)\left\|B_{i} \Lambda_{n}^{i-1} u_{n}-B_{i} p\right\|^{2} \\
& +2 \sigma_{n}\|(I-V) p\|\left\|z_{n}-p\right\|,
\end{aligned}
$$

which together with $\left\{\lambda_{i, n}\right\} \quad \subset \quad\left[a_{i}, b_{i}\right] \subset\left(0,2 \eta_{i}\right), \forall i \in$ $\{1,2, \ldots, N\}$, implies that

$$
\begin{aligned}
&(1-\widehat{a}) a_{i}\left(2 \eta_{i}-b_{i}\right)\left\|B_{i} \Lambda_{n}^{i-1} u_{n}-B_{i} p\right\|^{2} \\
& \leq\left(1-\beta_{n}\right) \lambda_{i, n}\left(2 \eta_{i}-\lambda_{i, n}\right)\left\|B_{i} \Lambda_{n}^{i-1} u_{n}-B_{i} p\right\|^{2} \\
& \leq\left\|x_{n}-p\right\|^{2}-\left\|z_{n}-p\right\|^{2}+2 \sigma_{n}\|(I-V) p\| \\
& \times\left\|z_{n}-p\right\| \\
& \leq\left\|x_{n}-z_{n}\right\|\left(\left\|x_{n}-p\right\|+\left\|z_{n}-p\right\|\right) \\
&+2 \sigma_{n}\|(I-V) p\|\left\|z_{n}-p\right\| .
\end{aligned}
$$

Since $\lim _{n \rightarrow \infty} \sigma_{n}=0$ and $\left\{x_{n}\right\}$ and $\left\{z_{n}\right\}$ are bounded sequences, it follows from (77) that

$$
\lim _{n \rightarrow \infty}\left\|B_{i} \Lambda_{n}^{i-1} u_{n}-B_{i} p\right\|=0, \quad i=1,2, \ldots, N
$$

By Proposition 5(iii) and Lemma 9(a), we obtain

$$
\begin{aligned}
& \left\|\Lambda_{n}^{i} u_{n}-p\right\|^{2} \\
& \quad=\left\|P_{C}\left(I-\lambda_{i, n} B_{i}\right) \Lambda_{n}^{i-1} u_{n}-P_{C}\left(I-\lambda_{i, n} B_{i}\right) p\right\|^{2} \\
& \quad \leq\left\langle\left(I-\lambda_{i, n} B_{i}\right) \Lambda_{n}^{i-1} u_{n}-\left(I-\lambda_{i, n} B_{i}\right) p, \Lambda_{n}^{i} u_{n}-p\right\rangle
\end{aligned}
$$


14

Abstract and Applied Analysis

$$
\begin{aligned}
& =\frac{1}{2}\left(\left\|\left(I-\lambda_{i, n} B_{i}\right) \Lambda_{n}^{i-1} u_{n}-\left(I-\lambda_{i, n} B_{i}\right) p\right\|^{2}\right. \\
& +\left\|\Lambda_{n}^{i} u_{n}-p\right\|^{2}-\|\left(I-\lambda_{i, n} B_{i}\right) \Lambda_{n}^{i-1} u_{n} \\
& \left.-\left(I-\lambda_{i, n} B_{i}\right) p-\left(\Lambda_{n}^{i} u_{n}-p\right) \|^{2}\right) \\
& \leq \frac{1}{2}\left(\left\|\Lambda_{n}^{i-1} u_{n}-p\right\|^{2}+\left\|\Lambda_{n}^{i} u_{n}-p\right\|^{2}\right. \\
& \left.-\left\|\Lambda_{n}^{i-1} u_{n}-\Lambda_{n}^{i} u_{n}-\lambda_{i, n}\left(B_{i} \Lambda_{n}^{i-1} u_{n}-B_{i} p\right)\right\|^{2}\right) \\
& \leq \frac{1}{2}\left(\left\|u_{n}-p\right\|^{2}+\left\|\Lambda_{n}^{i} u_{n}-p\right\|^{2}\right. \\
& \left.-\left\|\Lambda_{n}^{i-1} u_{n}-\Lambda_{n}^{i} u_{n}-\lambda_{i, n}\left(B_{i} \Lambda_{n}^{i-1} u_{n}-B_{i} p\right)\right\|^{2}\right) \\
& \leq \frac{1}{2}\left(\left\|x_{n}-p\right\|^{2}+\left\|\Lambda_{n}^{i} u_{n}-p\right\|^{2}\right. \\
& \left.-\left\|\Lambda_{n}^{i-1} u_{n}-\Lambda_{n}^{i} u_{n}-\lambda_{i, n}\left(B_{i} \Lambda_{n}^{i-1} u_{n}-B_{i} p\right)\right\|^{2}\right),
\end{aligned}
$$

which implies

$$
\begin{aligned}
&\left\|\Lambda_{n}^{i} u_{n}-p\right\|^{2} \\
& \leq\left\|x_{n}-p\right\|^{2} \\
& \quad-\left\|\Lambda_{n}^{i-1} u_{n}-\Lambda_{n}^{i} u_{n}-\lambda_{i, n}\left(B_{i} \Lambda_{n}^{i-1} u_{n}-B_{i} p\right)\right\|^{2} \\
&=\left\|x_{n}-p\right\|^{2}-\left\|\Lambda_{n}^{i-1} u_{n}-\Lambda_{n}^{i} u_{n}\right\|^{2} \\
& \quad-\lambda_{i, n}^{2}\left\|B_{i} \Lambda_{n}^{i-1} u_{n}-B_{i} p\right\|^{2} \\
& \quad+2 \lambda_{i, n}\left\langle\Lambda_{n}^{i-1} u_{n}-\Lambda_{n}^{i} u_{n}, B_{i} \Lambda_{n}^{i-1} u_{n}-B_{i} p\right\rangle \\
& \leq\left\|x_{n}-p\right\|^{2}-\left\|\Lambda_{n}^{i-1} u_{n}-\Lambda_{n}^{i} u_{n}\right\|^{2} \\
&+2 \lambda_{i, n}\left\|\Lambda_{n}^{i-1} u_{n}-\Lambda_{n}^{i} u_{n}\right\|\left\|B_{i} \Lambda_{n}^{i-1} u_{n}-B_{i} p\right\| .
\end{aligned}
$$

Combining (59), (82), and (97), we have

$$
\begin{aligned}
\| z_{n}- & p \|^{2} \\
\leq & \beta_{n}\left\|x_{n}-p\right\|^{2}+\left(1-\beta_{n}\right)\left\|v_{n}-p\right\|^{2} \\
& +2 \sigma_{n}\|(I-V) p\|\left\|z_{n}-p\right\| \\
\leq & \beta_{n}\left\|x_{n}-p\right\|^{2}+\left(1-\beta_{n}\right)\left\|\Lambda_{n}^{i} u_{n}-p\right\|^{2} \\
& +2 \sigma_{n}\|(I-V) p\|\left\|z_{n}-p\right\| \\
\leq & \beta_{n}\left\|x_{n}-p\right\|^{2}+\left(1-\beta_{n}\right) \\
\quad & \times\left[\left\|x_{n}-p\right\|^{2}-\left\|\Lambda_{n}^{i-1} u_{n}-\Lambda_{n}^{i} u_{n}\right\|^{2}\right.
\end{aligned}
$$

$$
\begin{aligned}
& \left.\quad+2 \lambda_{i, n}\left\|\Lambda_{n}^{i-1} u_{n}-\Lambda_{n}^{i} u_{n}\right\|\left\|B_{i} \Lambda_{n}^{i-1} u_{n}-B_{i} p\right\|\right] \\
& +2 \sigma_{n}\|(I-V) p\|\left\|z_{n}-p\right\| \\
& \leq\left\|x_{n}-p\right\|^{2}-\left(1-\beta_{n}\right)\left\|\Lambda_{n}^{i-1} u_{n}-\Lambda_{n}^{i} u_{n}\right\|^{2} \\
& +2 \lambda_{i, n}\left\|\Lambda_{n}^{i-1} u_{n}-\Lambda_{n}^{i} u_{n}\right\|\left\|B_{i} \Lambda_{n}^{i-1} u_{n}-B_{i} p\right\| \\
& +2 \sigma_{n}\|(I-V) p\|\left\|z_{n}-p\right\| .
\end{aligned}
$$

So, we conclude that

$$
\begin{aligned}
(1-\widehat{a}) & \left\|\Lambda_{n}^{i-1} u_{n}-\Lambda_{n}^{i} u_{n}\right\|^{2} \\
\leq & \left(1-\beta_{n}\right)\left\|\Lambda_{n}^{i-1} u_{n}-\Lambda_{n}^{i} u_{n}\right\|^{2} \\
\leq & \left\|x_{n}-p\right\|^{2}-\left\|z_{n}-p\right\|^{2} \\
& +2 \lambda_{i, n}\left\|\Lambda_{n}^{i-1} u_{n}-\Lambda_{n}^{i} u_{n}\right\|\left\|B_{i} \Lambda_{n}^{i-1} u_{n}-B_{i} p\right\| \\
& +2 \sigma_{n}\|(I-V) p\|\left\|z_{n}-p\right\| \\
\leq & \left\|x_{n}-z_{n}\right\|\left(\left\|x_{n}-p\right\|+\left\|z_{n}-p\right\|\right) \\
& +2 b_{i}\left\|\Lambda_{n}^{i-1} u_{n}-\Lambda_{n}^{i} u_{n}\right\|\left\|B_{i} \Lambda_{n}^{i-1} u_{n}-B_{i} p\right\| \\
& +2 \sigma_{n}\|(I-V) p\|\left\|z_{n}-p\right\| .
\end{aligned}
$$

Since $\lim _{n \rightarrow \infty} \sigma_{n}=0$ and $\left\{x_{n}\right\},\left\{z_{n}\right\}$, and $\left\{u_{n}\right\}$ are bounded, from (77) and (95) we get

$$
\lim _{n \rightarrow \infty}\left\|\Lambda_{n}^{i-1} u_{n}-\Lambda_{n}^{i} u_{n}\right\|=0
$$

From (100) we get

$$
\begin{aligned}
& \left\|u_{n}-v_{n}\right\| \\
& =\left\|\Lambda_{n}^{0} u_{n}-\Lambda_{n}^{N} u_{n}\right\| \\
& \leq\left\|\Lambda_{n}^{0} u_{n}-\Lambda_{n}^{1} u_{n}\right\|+\left\|\Lambda_{n}^{1} u_{n}-\Lambda_{n}^{2} u_{n}\right\| \\
& +\cdots+\left\|\Lambda_{n}^{N-1} u_{n}-\Lambda_{n}^{N} u_{n}\right\| \longrightarrow 0 \\
& \text { as } n \longrightarrow \infty .
\end{aligned}
$$

Taking into account that $\left\|x_{n}-v_{n}\right\| \leq\left\|x_{n}-u_{n}\right\|+\left\|u_{n}-v_{n}\right\|$, we conclude from (83) and (101) that

$$
\lim _{n \rightarrow \infty}\left\|x_{n}-v_{n}\right\|=0 .
$$

On the other hand, for simplicity, we write $\widetilde{p}=T_{v_{2}}^{\Theta_{2}}(I-$ $\left.v_{2} A_{2}\right) p, \widetilde{v}_{n}=T_{v_{2}}^{\Theta_{2}}\left(I-v_{2} A_{2}\right) v_{n}$, and $w_{n}=G v_{n}=T_{v_{1}}^{\Theta_{1}}(I-$ $\left.\nu_{1} A_{1}\right) \widetilde{v}_{n}$ for all $n \geq 1$. Then

$$
\begin{aligned}
p & =G p=T_{v_{1}}^{\Theta_{1}}\left(I-v_{1} A_{1}\right) \tilde{p} \\
& =T_{v_{1}}^{\Theta_{1}}\left(I-v_{1} A_{1}\right) T_{v_{2}}^{\Theta_{2}}\left(I-v_{2} A_{2}\right) p .
\end{aligned}
$$


We now show that $\lim _{n \rightarrow \infty}\left\|G v_{n}-v_{n}\right\|=0$; that is, $\lim _{n \rightarrow \infty}\left\|w_{n}-v_{n}\right\|=0$. As a matter of fact, for $p \in \Omega$, it follows from (58), (59), and (82) that

$$
\begin{aligned}
\| z_{n}- & p \|^{2} \\
\leq & \beta_{n}\left\|x_{n}-p\right\|^{2}+\left(1-\beta_{n}\right)\left\|G v_{n}-p\right\|^{2} \\
& +2 \sigma_{n}\|(I-V) p\|\left\|z_{n}-p\right\| \\
= & \beta_{n}\left\|x_{n}-p\right\|^{2}+\left(1-\beta_{n}\right)\left\|w_{n}-p\right\|^{2} \\
& +2 \sigma_{n}\|(I-V) p\|\left\|z_{n}-p\right\| \\
\leq & \beta_{n}\left\|x_{n}-p\right\|^{2}+\left(1-\beta_{n}\right) \\
& \times\left[\left\|\widetilde{v}_{n}-\tilde{p}\right\|^{2}+v_{1}\left(v_{1}-2 \zeta_{1}\right)\left\|A_{1} \widetilde{v}_{n}-A_{1} \tilde{p}\right\|^{2}\right] \\
& +2 \sigma_{n}\|(I-V) p\|\left\|z_{n}-p\right\| \\
\leq & \beta_{n}\left\|x_{n}-p\right\|^{2}+\left(1-\beta_{n}\right) \\
& \times\left[\left\|v_{n}-p\right\|^{2}+v_{2}\left(v_{2}-2 \zeta_{2}\right)\left\|A_{2} v_{n}-A_{2} p\right\|^{2}\right. \\
& \left.+v_{1}\left(v_{1}-2 \zeta_{1}\right)\left\|A_{1} \widetilde{v}_{n}-A_{1} \tilde{p}\right\|^{2}\right] \\
& +2 \sigma_{n}\|(I-V) p\|\left\|z_{n}-p\right\| \\
\leq & \beta_{n}\left\|x_{n}-p\right\|^{2}+\left(1-\beta_{n}\right) \\
& \times\left[\left\|x_{n}-p\right\|^{2}+v_{2}\left(v_{2}-2 \zeta_{2}\right)\left\|A_{2} v_{n}-A_{2} p\right\|^{2}\right. \\
& \left.+v_{1}\left(v_{1}-2 \zeta_{1}\right)\left\|A_{1} \widetilde{v}_{n}-A_{1} \tilde{p}\right\|^{2}\right] \\
+ & 2 \sigma_{n}\|(I-V) p\|\left\|z_{n}-p\right\| \\
= & \left\|x_{n}-p\right\|^{2}+\left(1-\beta_{n}\right) \\
& \times\left[v_{2}\left(v_{2}-2 \zeta_{2}\right)\left\|A_{2} v_{n}-A_{2} p\right\|^{2}\right. \\
& \left.+2 \sigma_{n}\left(v_{1}-2 \zeta_{1}\right)\left\|A_{1} \widetilde{v}_{n}-A_{1} \tilde{p}\right\|^{2}\right] \\
&
\end{aligned}
$$

which immediately yields

$$
\begin{gathered}
(1-\widehat{a})\left[v_{2}\left(2 \zeta_{2}-v_{2}\right)\left\|A_{2} v_{n}-A_{2} p\right\|^{2}\right. \\
\left.+v_{1}\left(2 \zeta_{1}-v_{1}\right)\left\|A_{1} \widetilde{v}_{n}-A_{1} \widetilde{p}\right\|^{2}\right] \\
\leq\left(1-\beta_{n}\right)\left[v_{2}\left(2 \zeta_{2}-v_{2}\right)\left\|A_{2} v_{n}-A_{2} p\right\|^{2}\right. \\
\left.\quad+v_{1}\left(2 \zeta_{1}-v_{1}\right)\left\|A_{1} \widetilde{v}_{n}-A_{1} \widetilde{p}\right\|^{2}\right] \\
\leq\left\|x_{n}-p\right\|^{2}-\left\|z_{n}-p\right\|^{2}+2 \sigma_{n}\|(I-V) p\|\left\|z_{n}-p\right\| \\
\leq\left\|x_{n}-z_{n}\right\|\left(\left\|x_{n}-p\right\|+\left\|z_{n}-p\right\|\right) \\
+2 \sigma_{n}\|(I-V) p\|\left\|z_{n}-p\right\| .
\end{gathered}
$$

Since $\lim _{n \rightarrow \infty} \sigma_{n}=0$ and $\left\{x_{n}\right\}$ and $\left\{z_{n}\right\}$ are bounded, from (77) we get

$$
\lim _{n \rightarrow \infty}\left\|A_{2} v_{n}-A_{2} p\right\|=0, \quad \lim _{n \rightarrow \infty}\left\|A_{1} \widetilde{v}_{n}-A_{1} \tilde{p}\right\|=0 .
$$

Also, in terms of the firm nonexpansivity of $T_{\nu_{k}}^{\Theta_{k}}$ and the $\zeta_{k}$ inverse strong monotonicity of $A_{k}$ for $k=1,2$, we obtain from $v_{k} \in\left(0,2 \zeta_{k}\right), k \in\{1,2\}$, and (59) that

$$
\begin{aligned}
& \left\|\widetilde{v}_{n}-\widetilde{p}\right\|^{2} \\
& =\left\|T_{v_{2}}^{\Theta_{2}}\left(I-v_{2} A_{2}\right) v_{n}-T_{v_{2}}^{\Theta_{2}}\left(I-v_{2} A_{2}\right) p\right\|^{2} \\
& \leq\left\langle\left(I-v_{2} A_{2}\right) v_{n}-\left(I-v_{2} A_{2}\right) p, \widetilde{v}_{n}-\tilde{p}\right\rangle \\
& =\frac{1}{2}\left[\left\|\left(I-v_{2} A_{2}\right) v_{n}-\left(I-v_{2} A_{2}\right) p\right\|^{2}+\left\|\widetilde{v}_{n}-\tilde{p}\right\|^{2}\right. \\
& \left.-\left\|\left(I-v_{2} A_{2}\right) v_{n}-\left(I-v_{2} A_{2}\right) p-\left(\widetilde{v}_{n}-\widetilde{p}\right)\right\|^{2}\right] \\
& \leq \frac{1}{2}\left[\left\|v_{n}-p\right\|^{2}+\left\|\widetilde{v}_{n}-\tilde{p}\right\|^{2}\right. \\
& \left.-\left\|\left(v_{n}-\widetilde{v}_{n}\right)-v_{2}\left(A_{2} v_{n}-A_{2} p\right)-(p-\widetilde{p})\right\|^{2}\right] \\
& =\frac{1}{2}\left[\left\|v_{n}-p\right\|^{2}+\left\|\widetilde{v}_{n}-\widetilde{p}\right\|^{2}-\left\|\left(v_{n}-\widetilde{v}_{n}\right)-(p-\widetilde{p})\right\|^{2}\right. \\
& +2 v_{2}\left\langle\left(v_{n}-\widetilde{v}_{n}\right)-(p-\widetilde{p}), A_{2} v_{n}-A_{2} p\right\rangle \\
& \left.-v_{2}^{2}\left\|A_{2} v_{n}-A_{2} p\right\|^{2}\right] \\
& \left\|w_{n}-p\right\|^{2} \\
& =\left\|T_{v_{1}}^{\Theta_{1}}\left(I-v_{1} A_{1}\right) \widetilde{v}_{n}-T_{v_{1}}^{\Theta_{1}}\left(I-v_{1} A_{1}\right) \tilde{p}\right\|^{2} \\
& \leq\left\langle\left(I-v_{1} A_{1}\right) \widetilde{v}_{n}-\left(I-v_{1} A_{1}\right) \tilde{p}, w_{n}-p\right\rangle \\
& =\frac{1}{2}\left[\left\|\left(I-v_{1} A_{1}\right) \widetilde{v}_{n}-\left(I-v_{1} A_{1}\right) \tilde{p}\right\|^{2}+\left\|w_{n}-p\right\|^{2}\right. \\
& \left.-\left\|\left(I-v_{1} A_{1}\right) \widetilde{v}_{n}-\left(I-v_{1} A_{1}\right) \tilde{p}-\left(w_{n}-p\right)\right\|^{2}\right] \\
& \leq \frac{1}{2}\left[\left\|\widetilde{v}_{n}-\widetilde{p}\right\|^{2}+\left\|w_{n}-p\right\|^{2}-\left\|\left(\widetilde{v}_{n}-w_{n}\right)+(p-\widetilde{p})\right\|^{2}\right. \\
& +2 v_{1}\left\langle A_{1} \widetilde{v}_{n}-A_{1} \widetilde{p},\left(\widetilde{v}_{n}-w_{n}\right)+(p-\widetilde{p})\right\rangle \\
& \left.-v_{1}^{2}\left\|A_{1} \widetilde{v}_{n}-A_{1} \tilde{p}\right\|^{2}\right] \\
& \leq \frac{1}{2}\left[\left\|v_{n}-p\right\|^{2}+\left\|w_{n}-p\right\|^{2}-\left\|\left(\widetilde{v}_{n}-w_{n}\right)+(p-\widetilde{p})\right\|^{2}\right. \\
& \left.+2 v_{1}\left\langle A_{1} \widetilde{v}_{n}-A_{1} \tilde{p},\left(\widetilde{v}_{n}-w_{n}\right)+(p-\widetilde{p})\right\rangle\right] .
\end{aligned}
$$


Thus, we have

$$
\begin{aligned}
\left\|\widetilde{v}_{n}-\tilde{p}\right\|^{2} \leq & \left\|v_{n}-p\right\|^{2}-\left\|\left(v_{n}-\widetilde{v}_{n}\right)-(p-\widetilde{p})\right\|^{2} \\
& +2 v_{2}\left\langle\left(v_{n}-\widetilde{v}_{n}\right)-(p-\widetilde{p}), A_{2} v_{n}-A_{2} p\right\rangle \\
& -v_{2}^{2}\left\|A_{2} v_{n}-A_{2} p\right\|^{2},
\end{aligned}
$$

$$
\begin{aligned}
\left\|w_{n}-p\right\|^{2} \leq & \left\|v_{n}-p\right\|^{2}-\left\|\left(\widetilde{v}_{n}-w_{n}\right)+(p-\widetilde{p})\right\|^{2} \\
& +2 v_{1}\left\|A_{1} \widetilde{v}_{n}-A_{1} \tilde{p}\right\|\left\|\left(\widetilde{v}_{n}-w_{n}\right)+(p-\widetilde{p})\right\| .
\end{aligned}
$$

Consequently, from (58), (104), and (108) it follows that

$$
\begin{aligned}
& \left\|z_{n}-p\right\|^{2} \\
& \leq \beta_{n}\left\|x_{n}-p\right\|^{2}+\left(1-\beta_{n}\right) \\
& \times\left[\left\|\widetilde{v}_{n}-\tilde{p}\right\|^{2}+v_{1}\left(\nu_{1}-2 \zeta_{1}\right)\left\|A_{1} \widetilde{v}_{n}-A_{1} \tilde{p}\right\|^{2}\right] \\
& +2 \sigma_{n}\|(I-V) p\|\left\|z_{n}-p\right\| \\
& \leq \beta_{n}\left\|x_{n}-p\right\|^{2}+\left(1-\beta_{n}\right)\left\|\widetilde{v}_{n}-\tilde{p}\right\|^{2} \\
& +2 \sigma_{n}\|(I-V) p\|\left\|z_{n}-p\right\| \\
& \leq \beta_{n}\left\|x_{n}-p\right\|^{2}+\left(1-\beta_{n}\right) \\
& \times\left[\left\|v_{n}-p\right\|^{2}-\left\|\left(v_{n}-\widetilde{v}_{n}\right)-(p-\widetilde{p})\right\|^{2}\right. \\
& +2 v_{2}\left\langle\left(v_{n}-\widetilde{v}_{n}\right)-(p-\tilde{p}), A_{2} v_{n}-A_{2} p\right\rangle \\
& \left.-v_{2}^{2}\left\|A_{2} v_{n}-A_{2} p\right\|^{2}\right] \\
& +2 \sigma_{n}\|(I-V) p\|\left\|z_{n}-p\right\| \\
& \leq \beta_{n}\left\|x_{n}-p\right\|^{2}+\left(1-\beta_{n}\right) \\
& \times\left[\left\|x_{n}-p\right\|^{2}-\left\|\left(v_{n}-\widetilde{v}_{n}\right)-(p-\tilde{p})\right\|^{2}\right. \\
& \left.+2 v_{2}\left\|\left(v_{n}-\widetilde{v}_{n}\right)-(p-\tilde{p})\right\|\left\|A_{2} v_{n}-A_{2} p\right\|\right] \\
& +2 \sigma_{n}\|(I-V) p\|\left\|z_{n}-p\right\| \\
& \leq\left\|x_{n}-p\right\|^{2}-\left(1-\beta_{n}\right)\left\|\left(v_{n}-\widetilde{v}_{n}\right)-(p-\widetilde{p})\right\|^{2} \\
& +2 v_{2}\left\|\left(v_{n}-\widetilde{v}_{n}\right)-(p-\widetilde{p})\right\|\left\|A_{2} v_{n}-A_{2} p\right\| \\
& +2 \sigma_{n}\|(I-V) p\|\left\|z_{n}-p\right\| \text {, }
\end{aligned}
$$

which hence leads to

$$
\begin{aligned}
& (1-\widehat{a})\left\|\left(v_{n}-\widetilde{v}_{n}\right)-(p-\widetilde{p})\right\|^{2} \\
& \quad \leq\left(1-\beta_{n}\right)\left\|\left(v_{n}-\widetilde{v}_{n}\right)-(p-\widetilde{p})\right\|^{2} \\
& \quad \leq\left\|x_{n}-p\right\|^{2}-\left\|z_{n}-p\right\|^{2}
\end{aligned}
$$

$$
\begin{aligned}
& +2 v_{2}\left\|\left(v_{n}-\widetilde{v}_{n}\right)-(p-\widetilde{p})\right\|\left\|A_{2} v_{n}-A_{2} p\right\| \\
& +2 \sigma_{n}\|(I-V) p\|\left\|z_{n}-p\right\| \\
\leq & \left\|x_{n}-z_{n}\right\|\left(\left\|x_{n}-p\right\|+\left\|z_{n}-p\right\|\right) \\
& +2 v_{2}\left\|\left(v_{n}-\widetilde{v}_{n}\right)-(p-\widetilde{p})\right\|\left\|A_{2} v_{n}-A_{2} p\right\| \\
& +2 \sigma_{n}\|(I-V) p\|\left\|z_{n}-p\right\| .
\end{aligned}
$$

Since $\lim _{n \rightarrow \infty} \sigma_{n}=0$ and $\left\{x_{n}\right\},\left\{z_{n}\right\},\left\{v_{n}\right\}$, and $\left\{\widetilde{v}_{n}\right\}$ are bounded sequences, we conclude from (77) and (106) that

$$
\lim _{n \rightarrow \infty}\left\|\left(v_{n}-\widetilde{v}_{n}\right)-(p-\tilde{p})\right\|=0 .
$$

Furthermore, from (58), (104), and (109) it follows that

$$
\begin{aligned}
\| z_{n}- & p \|^{2} \\
\leq & \beta_{n}\left\|x_{n}-p\right\|^{2}+\left(1-\beta_{n}\right)\left\|w_{n}-p\right\|^{2} \\
& +2 \sigma_{n}\|(I-V) p\|\left\|z_{n}-p\right\| \\
\leq & \beta_{n}\left\|x_{n}-p\right\|^{2}+\left(1-\beta_{n}\right) \\
& \times\left[\left\|v_{n}-p\right\|^{2}-\left\|\left(\widetilde{v}_{n}-w_{n}\right)+(p-\widetilde{p})\right\|^{2}\right. \\
& \left.+2 v_{1}\left\|A_{1} \widetilde{v}_{n}-A_{1} \tilde{p}\right\|\left\|\left(\widetilde{v}_{n}-w_{n}\right)+(p-\widetilde{p})\right\|\right] \\
& +2 \sigma_{n}\|(I-V) p\|\left\|z_{n}-p\right\| \\
\leq & \beta_{n}\left\|x_{n}-p\right\|^{2}+\left(1-\beta_{n}\right) \\
& \times\left[\left\|x_{n}-p\right\|^{2}-\left\|\left(\widetilde{v}_{n}-w_{n}\right)+(p-\widetilde{p})\right\|^{2}\right. \\
& \left.+2 v_{1}\left\|A_{1} \widetilde{v}_{n}-A_{1} \tilde{p}\right\|\left\|\left(\widetilde{v}_{n}-w_{n}\right)+(p-\widetilde{p})\right\|\right] \\
& +2 \sigma_{n}\|(I-V) p\|\left\|z_{n}-p\right\| \\
\leq & \left\|x_{n}-p\right\|^{2}-\left(1-\beta_{n}\right)\left\|\left(\widetilde{v}_{n}-w_{n}\right)+(p-\widetilde{p})\right\|^{2} \\
& +2 v_{1}\left\|A_{1} \widetilde{v}_{n}-A_{1} \tilde{p}\right\|\left\|\left(\widetilde{v}_{n}-w_{n}\right)+(p-\widetilde{p})\right\| \\
& +2 \sigma_{n}\|(I-V) p\|\left\|z_{n}-p\right\|,
\end{aligned}
$$

which hence yields

$$
\begin{aligned}
(1-\widehat{a}) & \left\|\left(\widetilde{v}_{n}-w_{n}\right)+(p-\tilde{p})\right\|^{2} \\
\leq & \left(1-\beta_{n}\right)\left\|\left(\widetilde{v}_{n}-w_{n}\right)+(p-\widetilde{p})\right\|^{2} \\
\leq & \left\|x_{n}-p\right\|^{2}-\left\|z_{n}-p\right\|^{2}+2 v_{1}\left\|A_{1} \widetilde{v}_{n}-A_{1} \widetilde{p}\right\| \\
& \times\left\|\left(\widetilde{v}_{n}-w_{n}\right)+(p-\widetilde{p})\right\| \\
& +2 \sigma_{n}\|(I-V) p\|\left\|z_{n}-p\right\| \\
\leq & \left\|x_{n}-z_{n}\right\|\left(\left\|x_{n}-p\right\|+\left\|z_{n}-p\right\|\right) \\
& +2 v_{1}\left\|A_{1} \widetilde{v}_{n}-A_{1} \tilde{p}\right\|\left\|\left(\widetilde{v}_{n}-w_{n}\right)+(p-\widetilde{p})\right\| \\
& +2 \sigma_{n}\|(I-V) p\|\left\|z_{n}-p\right\| .
\end{aligned}
$$


Since $\lim _{n \rightarrow \infty} \sigma_{n}=0$ and $\left\{x_{n}\right\},\left\{z_{n}\right\},\left\{w_{n}\right\}$, and $\left\{\widetilde{v}_{n}\right\}$ are bounded sequences, we conclude from (77) and (106) that

$$
\lim _{n \rightarrow \infty}\left\|\left(\widetilde{v}_{n}-w_{n}\right)+(p-\widetilde{p})\right\|=0 .
$$

Note that

$$
\left\|v_{n}-w_{n}\right\| \leq\left\|\left(v_{n}-\widetilde{v}_{n}\right)-(p-\tilde{p})\right\|+\left\|\left(\widetilde{v}_{n}-w_{n}\right)+(p-\tilde{p})\right\| .
$$

Hence from (112) and (115) we get

$$
\lim _{n \rightarrow \infty}\left\|v_{n}-G v_{n}\right\|=\lim _{n \rightarrow \infty}\left\|v_{n}-w_{n}\right\|=0,
$$

then by (75), (102), and (117), we have

$$
\begin{aligned}
& \left\|x_{n}-W_{n} x_{n}\right\| \\
& \quad \leq\left\|x_{n}-W_{n} G v_{n}\right\|+\left\|W_{n} G v_{n}-W_{n} x_{n}\right\| \\
& \quad \leq\left\|x_{n}-W_{n} G v_{n}\right\|+\left\|G v_{n}-x_{n}\right\| \\
& \quad \leq\left\|x_{n}-W_{n} G v_{n}\right\|+\left\|G v_{n}-v_{n}\right\|+\left\|v_{n}-x_{n}\right\| \rightarrow 0
\end{aligned}
$$$$
\text { as } n \longrightarrow \infty
$$

Also, observe that

$$
\left\|x_{n}-W x_{n}\right\| \leq\left\|x_{n}-W_{n} x_{n}\right\|+\left\|W_{n} x_{n}-W x_{n}\right\| .
$$

From (118), [45, Remark 3.2], and the boundedness of $\left\{x_{n}\right\}$ we immediately obtain

$$
\lim _{n \rightarrow \infty}\left\|x_{n}-W x_{n}\right\|=0 .
$$

In addition, from (67) and (77), we have

$$
\begin{aligned}
& \left\|z_{n+1}-z_{n}\right\| \\
& \quad \leq\left\|z_{n+1}-x_{n+1}\right\|+\left\|x_{n+1}-x_{n}\right\|+\left\|x_{n}-z_{n}\right\| \longrightarrow 0
\end{aligned}
$$

as $n \longrightarrow \infty$.

We note that

$$
\begin{aligned}
\left\|S^{n} z_{n}-S^{n+1} z_{n}\right\| \leq & \left\|S^{n} z_{n}-z_{n}\right\|+\left\|z_{n}-z_{n+1}\right\| \\
& +\left\|z_{n+1}-S^{n+1} z_{n+1}\right\| \\
& +\left\|S^{n+1} z_{n+1}-S^{n+1} z_{n}\right\| .
\end{aligned}
$$

From (81), (121), and Lemma 15, we obtain

$$
\lim _{n \rightarrow \infty}\left\|S^{n} z_{n}-S^{n+1} z_{n}\right\|=0
$$

In the meantime, we note that

$$
\begin{aligned}
\left\|z_{n}-S z_{n}\right\| \leq & \left\|z_{n}-S^{n} z_{n}\right\|+\left\|S^{n} z_{n}-S^{n+1} z_{n}\right\| \\
& +\left\|S^{n+1} z_{n}-S z_{n}\right\| .
\end{aligned}
$$

From (81), (123), and the uniform continuity of $S$, we have

$$
\lim _{n \rightarrow \infty}\left\|z_{n}-S z_{n}\right\|=0 .
$$

Step 4. We prove that $x_{n} \rightarrow x^{*}=P_{\Omega} x_{0}$ as $n \rightarrow \infty$.

Indeed, since $\left\{x_{n}\right\}$ is bounded, there exists a subsequence $\left\{x_{n_{i}}\right\}$ which converges weakly to some $w$. From (102), (83), (100), and (77), we have that $v_{n_{i}} \rightarrow w, u_{n_{i}} \rightarrow w, \Lambda_{n_{i}}^{m} u_{n_{i}} \rightarrow w$, and $z_{n_{i}} \rightarrow w$, where $m \in\{1,2, \ldots, N\}$. Since $S$ is uniformly continuous, by (125) we get $\lim _{n \rightarrow \infty}\left\|z_{n}-S^{m} z_{n}\right\|=0$ for any $m \geq 1$. Hence from Lemma 17, we obtain $w \in \operatorname{Fix}(S)$. In the meantime, utilizing Lemma 12, we deduce from $v_{n_{i}} \rightarrow$ $w, x_{n_{i}} \rightarrow w$, (117), and (120) that $w \in \operatorname{SGEP}(G)$ and $w \in$ $\operatorname{Fix}(W)=\cap_{n=1}^{\infty} \operatorname{Fix}\left(T_{n}\right)$ (due to Lemma 11). Next, we prove that $w \in \cap_{m=1}^{N} \operatorname{VI}\left(C, B_{m}\right)$. As a matter of fact, let

$$
\widetilde{T}_{m} v= \begin{cases}B_{m} v+N_{C} v, & v \in C, \\ \emptyset, & v \notin C,\end{cases}
$$

where $m \in\{1,2, \ldots, N\}$. Let $(v, u) \in G\left(\widetilde{T}_{m}\right)$. Since $u-B_{m} v \in$ $N_{C} v$ and $\Lambda_{n}^{m} u_{n} \in C$, we have

$$
\left\langle v-\Lambda_{n}^{m} u_{n}, u-B_{m} v\right\rangle \geq 0 .
$$

On the other hand, from $\Lambda_{n}^{m} u_{n}=P_{C}\left(I-\lambda_{m, n} B_{m}\right) \Lambda_{n}^{m-1} u_{n}$ and $v \in C$, we have

$$
\left\langle v-\Lambda_{n}^{m} u_{n}, \Lambda_{n}^{m} u_{n}-\left(\Lambda_{n}^{m-1} u_{n}-\lambda_{m, n} B_{m} \Lambda_{n}^{m-1} u_{n}\right)\right\rangle \geq 0,
$$

and hence

$$
\left\langle v-\Lambda_{n}^{m} u_{n}, \frac{\Lambda_{n}^{m} u_{n}-\Lambda_{n}^{m-1} u_{n}}{\lambda_{m, n}}+B_{m} \Lambda_{n}^{m-1} u_{n}\right\rangle \geq 0 .
$$

Therefore we have

$$
\begin{aligned}
\langle v- & \left.\Lambda_{n_{i}}^{m} u_{n_{i}}, u\right\rangle \\
\geq & \left\langle v-\Lambda_{n_{i}}^{m} u_{n_{i}}, B_{m} v\right\rangle \\
\geq & \left\langle v-\Lambda_{n_{i}}^{m} u_{n_{i}}, B_{m} v\right\rangle \\
& -\left\langle v-\Lambda_{n_{i}}^{m} u_{n_{i}}, \frac{\Lambda_{n_{i}}^{m} u_{n_{i}}-\Lambda_{n_{i}}^{m-1} u_{n_{i}}}{\lambda_{m, n_{i}}}+B_{m} \Lambda_{n_{i}}^{m-1} u_{n_{i}}\right\rangle \\
= & \left\langle v-\Lambda_{n_{i}}^{m} u_{n_{i}}, B_{m} v-B_{m} \Lambda_{n_{i}}^{m} u_{n_{i}}\right\rangle \\
& +\left\langle v-\Lambda_{n_{i}}^{m} u_{n_{i}}, B_{m} \Lambda_{n_{i}}^{m} u_{n_{i}}-B_{m} \Lambda_{n_{i}}^{m-1} u_{n_{i}}\right\rangle \\
& -\left\langle v-\Lambda_{n_{i}}^{m} u_{n_{i}}, \frac{\Lambda_{n_{i}}^{m} u_{n_{i}}-\Lambda_{n_{i}}^{m-1} u_{n_{i}}}{\lambda_{m, n_{i}}}\right\rangle \\
\geq & \left\langle v-\Lambda_{n_{i}}^{m} u_{n_{i}}, B_{m} \Lambda_{n_{i}}^{m} u_{n_{i}}-B_{m} \Lambda_{n_{i}}^{m-1} u_{n_{i}}\right\rangle \\
& -\left\langle v-\Lambda_{n_{i}}^{m} u_{n_{i}}, \frac{\Lambda_{n_{i}}^{m} u_{n_{i}}-\Lambda_{n_{i}}^{m-1} u_{n_{i}}}{\lambda_{m, n_{i}}}\right\rangle .
\end{aligned}
$$

From (100) and since $B_{m}$ is uniformly continuous, we obtain that $\lim _{n \rightarrow \infty}\left\|B_{m} \Lambda_{n}^{m} u_{n}-B_{m} \Lambda_{n}^{m-1} u_{n}\right\|=0$. From $\Lambda_{n_{i}}^{m} u_{n_{i}} \rightarrow w$, 
$\left\{\lambda_{m, n}\right\} \subset\left[a_{m}, b_{m}\right] \subset\left(0,2 \eta_{m}\right), \forall m \in\{1,2, \ldots, N\}$, and (100), we have

$$
\langle v-w, u\rangle \geq 0
$$

Since $\widetilde{T}_{m}$ is maximal monotone, we have $w \in \widetilde{T}_{m}^{-1} 0$ and hence $w \in \operatorname{VI}\left(C, B_{m}\right), m=1,2, \ldots, N$, which implies $w \in$ $\cap_{m=1}^{N} \operatorname{VI}\left(C, B_{m}\right)$.

Next, we show that $w \in \operatorname{GMEP}(\Theta, \varphi, A)$. In fact, from $u_{n}=S_{r_{n}}^{(\Theta, \varphi)}\left(I-r_{n} A\right) x_{n}$, we know that

$$
\begin{aligned}
& \Theta\left(u_{n}, y\right)+\varphi(y)-\varphi\left(u_{n}\right)+\left\langle A x_{n}, y-u_{n}\right\rangle \\
& +\frac{1}{r_{n}}\left\langle K^{\prime}\left(u_{n}\right)-K^{\prime}\left(x_{n}\right), y-u_{n}\right\rangle \geq 0, \quad \forall y \in C .
\end{aligned}
$$

From (H2) it follows that

$$
\begin{aligned}
& \varphi(y)-\varphi\left(u_{n}\right)+\left\langle A x_{n}, y-u_{n}\right\rangle \\
& \quad+\frac{1}{r_{n}}\left\langle K^{\prime}\left(u_{n}\right)-K^{\prime}\left(x_{n}\right), y-u_{n}\right\rangle \geq \Theta\left(y, u_{n}\right),
\end{aligned}
$$

$\forall y \in C$.

Replacing $n$ by $n_{i}$, we have

$$
\begin{aligned}
& \varphi(y)-\varphi\left(u_{n_{i}}\right)+\left\langle A x_{n_{i}}, y-u_{n_{i}}\right\rangle \\
& +\left\langle\frac{K^{\prime}\left(u_{n_{i}}\right)-K^{\prime}\left(x_{n_{i}}\right)}{r_{n_{i}}}, y-u_{n_{i}}\right\rangle \geq \Theta\left(y, u_{n_{i}}\right),
\end{aligned}
$$

$\forall y \in C$.

Put $u_{t}=t y+(1-t) w$ for all $t \in(0,1]$ and $y \in C$. Then from (134) we have

$$
\begin{aligned}
\left\langle u_{t}\right. & \left.-u_{n_{i}}, A u_{t}\right\rangle \\
\geq & \left\langle u_{t}-u_{n_{i}}, A u_{t}\right\rangle-\varphi\left(u_{t}\right)+\varphi\left(u_{n_{i}}\right)-\left\langle u_{t}-u_{n_{i}}, A x_{n_{i}}\right\rangle \\
& -\left\langle\frac{K^{\prime}\left(u_{n_{i}}\right)-K^{\prime}\left(x_{n_{i}}\right)}{r_{n_{i}}}, u_{t}-u_{n_{i}}\right\rangle+\Theta\left(u_{t}, u_{n_{i}}\right) \\
\geq & \left\langle u_{t}-u_{n_{i}}, A u_{t}-A u_{n_{i}}\right\rangle+\left\langle u_{t}-u_{n_{i}}, A u_{n_{i}}-A x_{n_{i}}\right\rangle \\
& -\varphi\left(u_{t}\right)+\varphi\left(u_{n_{i}}\right) \\
& -\left\langle\frac{K^{\prime}\left(u_{n_{i}}\right)-K^{\prime}\left(x_{n_{i}}\right)}{r_{n_{i}}}, u_{t}-u_{n_{i}}\right\rangle+\Theta\left(u_{t}, u_{n_{i}}\right) .
\end{aligned}
$$

Since $\left\|u_{n_{i}}-x_{n_{i}}\right\| \rightarrow 0$ as $i \rightarrow \infty$, we deduce from the Lipschitz continuity of $A$ and $K^{\prime}$ that $\left\|A u_{n_{i}}-A x_{n_{i}}\right\| \rightarrow 0$ and $\left\|K^{\prime}\left(u_{n_{i}}\right)-K^{\prime}\left(x_{n_{i}}\right)\right\| \rightarrow 0$ as $i \rightarrow \infty$. Further, from the monotonicity of $A$, we have $\left\langle u_{t}-u_{n_{i}}, A u_{t}-A u_{n_{i}}\right\rangle \geq 0$. So, from (H4), the weakly lower semicontinuity of $\varphi,\left(K^{\prime}\left(u_{n_{i}}\right)-\right.$ $\left.K^{\prime}\left(x_{n_{i}}\right)\right) / r_{n_{i}} \rightarrow 0$ and $u_{n_{i}} \rightarrow w$, we have

$$
\left\langle u_{t}-w, A u_{t}\right\rangle \geq-\varphi\left(u_{t}\right)+\varphi(w)+\Theta\left(u_{t}, w\right), \quad \text { as } i \longrightarrow \infty \text {. }
$$

From (H1), (H4), and (136) we also have

$$
\begin{aligned}
0= & \Theta\left(u_{t}, u_{t}\right)+\varphi\left(u_{t}\right)-\varphi\left(u_{t}\right) \\
\leq & t \Theta\left(u_{t}, y\right)+(1-t) \Theta\left(u_{t}, w\right) \\
& +t \varphi(y)+(1-t) \varphi(w)-\varphi\left(u_{t}\right) \\
= & t\left[\Theta\left(u_{t}, y\right)+\varphi(y)-\varphi\left(u_{t}\right)\right] \\
& +(1-t)\left[\Theta\left(u_{t}, w\right)+\varphi(w)-\varphi(w)-\varphi\left(u_{t}\right)\right] \\
\leq & t\left[\Theta\left(u_{t}, y\right)+\varphi(y)-\varphi\left(u_{t}\right)\right] \\
& +(1-t)\left\langle u_{t}-w, A u_{t}\right\rangle \\
= & t\left[\Theta\left(u_{t}, y\right)+\varphi(y)-\varphi\left(u_{t}\right)\right] \\
& +(1-t) t\left\langle y-w, A u_{t}\right\rangle,
\end{aligned}
$$

and hence

$$
0 \leq \Theta\left(u_{t}, y\right)+\varphi(y)-\varphi\left(u_{t}\right)+(1-t)\left\langle y-w, A u_{t}\right\rangle
$$

Letting $t \rightarrow 0^{+}$, we have, for each $y \in C$,

$$
0 \leq \Theta(w, y)+\varphi(y)-\varphi(w)+\langle A w, y-w\rangle
$$

This implies that $w \in \operatorname{GMEP}(\Theta, \varphi, A)$. Consequently, $w \in \Omega=\cap_{n=1}^{\infty} \operatorname{Fix}\left(T_{n}\right) \cap \operatorname{GMEP}(\Theta, \varphi, A) \cap \operatorname{SGEP}(G) \cap$ $\bigcap_{i=1}^{N} \operatorname{VI}\left(C, B_{i}\right) \cap \operatorname{Fix}(S)$. This shows that $\omega_{w}\left(x_{n}\right) \subset \Omega$. From (64) and Lemma 22 we infer that $x_{n} \rightarrow x^{*}=P_{\Omega} x_{0}$ as $n \rightarrow \infty$. This completes the proof.

Corollary 24. Choose $N=2$ in Theorem 23. For any $x_{0} \in H$, $C_{1}=C$, and $x_{1}=P_{C_{1}} x_{0}$, the iterative scheme (49) reduces to the following iterative one:

$$
\begin{gathered}
u_{n}=S_{r_{n}}^{(\Theta, \varphi)}\left(I-r_{n} A\right) x_{n}, \\
v_{n}=P_{C}\left(I-\lambda_{2, n} B_{2}\right) P_{C}\left(I-\lambda_{1, n} B_{1}\right) u_{n}, \\
z_{n}=\beta_{n} x_{n}+\sigma_{n} G v_{n}+\left[\left(1-\beta_{n}\right) I-\sigma_{n} V\right] W_{n} G v_{n}, \\
k_{n}=\delta_{n} z_{n}+\left(1-\delta_{n}\right) S^{n} z_{n}, \\
y_{n}=\left(1-\alpha_{n}\right) x_{n}+\alpha_{n} k_{n}, \\
C_{n+1}=\left\{z \in C_{n}:\left\|y_{n}-z\right\|^{2} \leq\left\|x_{n}-z\right\|^{2}+\theta_{n}\right\}, \\
x_{n+1}=P_{C_{n+1}} x_{0}, \quad \forall n \geq 1,
\end{gathered}
$$

where $\theta_{n}=\left(\sigma_{n}+\gamma_{n}\right)\left(1+\gamma_{n}\right) \Delta_{n}+c_{n}, \Delta_{n}=\sup \left\{\left\|x_{n}-p\right\|^{2}+\right.$ $\left.\|(I-V) p\|^{2} /(\bar{\gamma}-1): p \in \Omega\right\}<\infty, v_{k} \in\left(0,2 \zeta_{k}\right), k=$ 1,2 , and $\left\{\lambda_{i, n}\right\} \subset\left[a_{i}, b_{i}\right] \subset\left(0,2 \eta_{i}\right), i=1,2$. Then $\left\{x_{n}\right\}$ converges strongly to $x^{*}=P_{\Omega} x_{0}$ provided that $S_{r}^{(\Theta, \varphi)}$ is firmly nonexpansive. 
Corollary 25. Choose $N=1$ and $T_{n} \equiv I$ the identity operator of $H$ in Theorem 23. For any $x_{0} \in H, C_{1}=C$, and $x_{1}=P_{C_{1}} x_{0}$, the iterative scheme (49) reduces to the following iterative one:

$$
\begin{gathered}
u_{n}=S_{r_{n}}^{(\Theta, \varphi)}\left(I-r_{n} A\right) x_{n}, \\
v_{n}=P_{C}\left(I-\lambda_{1, n} B_{1}\right), \\
z_{n}=\beta_{n} x_{n}+\left(1-\beta_{n}\right) G v_{n}+\sigma_{n}(I-V) G v_{n}, \\
k_{n}=\delta_{n} z_{n}+\left(1-\delta_{n}\right) S^{n} z_{n}, \\
y_{n}=\left(1-\alpha_{n}\right) x_{n}+\alpha_{n} k_{n}, \\
C_{n+1}=\left\{z \in C_{n}:\left\|y_{n}-z\right\|^{2} \leq\left\|x_{n}-z\right\|^{2}+\theta_{n}\right\}, \\
x_{n+1}=P_{C_{n+1}} x_{0}, \quad \forall n \geq 1,
\end{gathered}
$$

where $\theta_{n}=\left(\sigma_{n}+\gamma_{n}\right)\left(1+\gamma_{n}\right) \Delta_{n}+c_{n}, \Delta_{n}=\sup \left\{\left\|x_{n}-p\right\|^{2}+\right.$ $\left.\|(I-V) p\|^{2} /(\bar{\gamma}-1): p \in \Omega\right\}<\infty, v_{k} \in\left(0,2 \zeta_{k}\right), k=1,2$, and $\left\{\lambda_{1, n}\right\} \subset\left[a_{1}, b_{1}\right] \subset\left(0,2 \eta_{1}\right)$. Then $\left\{x_{n}\right\}$ converges strongly to $x^{*}=P_{\Omega} x_{0}$ provided that $S_{r}^{(\Theta, \varphi)}$ is firmly nonexpansive.

Proof. In Theorem 23, putting $N=1$ and $T_{n} \equiv I$ the identity operator of $H$, we have $W_{n} \equiv I$. In this case, we get

$$
\begin{aligned}
z_{n} & =\beta_{n} x_{n}+\sigma_{n} G v_{n}+\left[\left(1-\beta_{n}\right) I-\sigma_{n} V\right] W_{n} G v_{n} \\
& =\beta_{n} x_{n}+\sigma_{n} G v_{n}+\left[\left(1-\beta_{n}\right) I-\sigma_{n} V\right] G v_{n} \\
& =\beta_{n} x_{n}+\left(1-\beta_{n}\right) G v_{n}+\sigma_{n}(I-V) G v_{n} .
\end{aligned}
$$

So, the iterative scheme (49) reduces to the iterative one (141). Utilizing Theorem 23, we derive the desired result.

Remark 26. Theorem 23 extends, improves, supplements, and develops Ceng et al.s [20, Theorem 1] in the following aspects.

(i) The problem of finding a point

$$
\begin{gathered}
x^{*} \in \bigcap_{i=1}^{N} \operatorname{Fix}\left(T_{n}\right) \cap \operatorname{GMEP}(\Theta, \varphi, A) \cap \\
\operatorname{SGEP}(G) \cap \bigcap_{i=1}^{N} \operatorname{VI}\left(C, B_{i}\right) \cap \operatorname{Fix}(S)
\end{gathered}
$$

in Theorem 23 is very different from the problem of finding a point

$$
x^{*} \in \cap_{n=1}^{\infty} \operatorname{Fix}\left(T_{n}\right) \cap \operatorname{GMEP}(\Theta, \varphi, A) \cap \operatorname{SGEP}(G)
$$

in Ceng et al.s [20, Theorem 1]. There is no doubt that our problem of finding a point $x^{*} \in \cap_{n=1}^{\infty} \operatorname{Fix}\left(T_{n}\right) \cap$ $\operatorname{GMEP}(\Theta, \varphi, A) \cap \operatorname{SGEP}(G) \cap \bigcap_{i=1}^{N} \operatorname{VI}\left(C, B_{i}\right) \cap \operatorname{Fix}(S)$ is more general and more subtle than the problem of finding a point $x^{*} \in \cap_{n=1}^{\infty} \operatorname{Fix}\left(T_{n}\right) \cap \operatorname{GMEP}(\Theta, \varphi, A) \cap \operatorname{SGEP}(G)$ in [20, Theorem 1].

(ii) The iterative scheme in [20, Theorem 1] is extended to develop the iterative scheme in Theorem 23 by the virtue of Mann-type iterative method and the shrinking projection method. The iterative scheme in Theorem 23 is more advantageous and more flexible than the iterative scheme in [20, Theorem 1] because it involves solving four problems: the GMEP (5), the SGEP (12), finitely many variational inequalities, and the common fixed point problem of an asymptotically strict pseudocontractive mapping in the intermediate sense and infinitely many nonexpansive mappings.

(iii) The iterative scheme in Theorem 23 is very different from the iterative scheme in [20, Theorem 1] because the iterative scheme in Theorem 23 involves Mann-type iterative method and the shrinking projection method. The proof of [20, Theorem 1] makes use of Lemma 12 (i.e., Demiclosedness principle for a nonexpansive mapping) but no use of Lemma 17 (i.e., Demiclosedness principle for an asymptotically strict pseudocontractive mapping in the intermediate sense). However, the proof of Theorem 23 depends on not only Lemma 12 but also Lemma 17 because there is an asymptotically strict pseudocontractive mapping in the intermediate sense and infinitely many nonexpansive mappings appearing in the problem of Theorem 23.

(iv) The proof of Theorem 23 combines Cai and $\mathrm{Bu}$ convergence analysis for Mann-type iterative method and the shrinking projection method to solve finitely many GMEPs, finitely many VIPs, and the fixed point problem of an asymptotically strict pseudocontractive mapping in the intermediate sense (see [26, Theorem 3.1]) and Ceng et al.s convergence analysis for hybrid extragradient-like iterative algorithm (see [20, Theorem 3.1]), where $\bar{\gamma} \in(0,1]$ for a $\bar{\gamma}$ strongly positive bounded linear operator $V$. Because in iterative scheme (49) the composite shrinking projection method involves a $\bar{\gamma}$-strongly positive bounded linear operator $V$ with $\bar{\gamma} \in(1,2]$ and infinitely many nonexpansive mappings, the properties of the $W$-mappings $W_{n}$ and $W$ and the operator $V$ play a key role in the proof of Theorem 23.

(v) Theorem 23 extends Ceng et al.s [20, Theorem 1] from the fixed point problem of infinitely many nonexpansive mappings to the common fixed point problem of an asymptotically strict pseudocontractive mapping in the intermediate sense and infinitely many nonexpansive mappings and generalizes Ceng et al.s [20, Theorem 1] to the setting of finitely many variational inequalities. The proof of Theorem 23 depends on the properties of the $\bar{\gamma}$-strongly positive bounded linear operator $V$ with $\bar{\gamma} \in(1,2]$, the result on the $W$-mappings $W_{n}$ and $W$ (i.e., $\lim _{n \rightarrow \infty}\left\|W_{n} x_{n}-W x_{n}\right\|=$ 0 for any bounded sequence $\left\{x_{n}\right\} \subset C$ ) (see [45, Remark 3.2]), and the properties of asymptotically strict pseudocontractive mapping in the intermediate sense (see Lemmas 15-18).

Remark 27. Theorem 23 extends, improves, supplements, and develops Yao et al.s [30, Theorem 3.1] in the following aspects.

(i) Theorem 23 generalizes and extends [30, Theorem 3.1] from the asymptotically $k$-strict pseudocontractive mapping to the asymptotically $k$-strict pseudocontractive mapping in the intermediate sense and from the MEP to the GMEP and generalizes [30, Theorem 3.1] to the setting of SGEP.

(ii) We add finitely many variational inequalities and infinitely many nonexpansive mappings $\left\{T_{n}\right\}_{n=1}^{\infty}$ 
in our algorithm such that it can be applied to find a common solution of the GMEP (5), the SGEP (12), finitely many variational inequalities for inverse strongly monotone mappings, and the common fixed point problem of an asymptotically $k$-strict pseudocontractive mapping in the intermediate sense and infinitely many nonexpansive mappings $\left\{T_{n}\right\}_{n=1}^{\infty}$.

\section{Weak Convergence Theorem}

In this section, we will propose and analyze another iterative algorithm (involving no shrinking projection method) for finding a solution of the system of generalized equilibria with constraints of several problems: a generalized mixed equilibrium problem, finitely many variational inequalities, and the common fixed point problem of an asymptotically strict pseudocontractive mapping in the intermediate sense and infinitely many nonexpansive mappings in a real Hilbert space. Moreover, under mild conditions we will prove weak convergence of the proposed algorithm.

Theorem 28. Let $C, N, \Theta, \Theta_{1}, \Theta_{2}, \varphi, A, A_{k}, B_{i}, V,\left\{T_{n}\right\}_{n=1}^{\infty}$, $\left\{\lambda_{n}\right\}$, and $W_{n}$ be the same notations as in Theorem 23, where $k \in\{1,2\}$ and $i \in\{1,2, \ldots, N\}$. Let $S: C \rightarrow C$ be a uniformly continuous asymptotically $k$-strict pseudocontractive mapping in the intermediate sense for some $0 \leq k<1$ with the sequence $\left\{\gamma_{n}\right\} \subset[0, \infty)$ such that $\sum_{n=1}^{\infty} \gamma_{n}<\infty$ and $\left\{c_{n}\right\} \subset[0, \infty)$ such that $\sum_{n=1}^{\infty} c_{n}<\infty$. Assume that $\Omega \bigcap_{i=1}^{N} \operatorname{Fix}\left(T_{n}\right) \cap$ $\operatorname{GMEP}(\Theta, \varphi, A) \cap \operatorname{SGEP}(G) \cap \bigcap_{i=1}^{N} \operatorname{VI}\left(C, B_{i}\right) \cap \operatorname{Fix}(S)$ is nonempty where $G$ is defined as in Proposition CY. Let $\left\{r_{n}\right\}$ be a sequence in $[0,2 \zeta]$ and let $\left\{\alpha_{n}\right\},\left\{\beta_{n}\right\},\left\{\sigma_{n}\right\}$, and $\left\{\delta_{n}\right\}$ be sequences in $[0,1]$ such that $\sum_{n=1}^{\infty} \sigma_{n}<\infty, 0<\alpha \leq \alpha_{n} \leq 1$, and $0<k+\epsilon \leq \delta_{n} \leq d<1$. Pick any $x_{1} \in H$ and let $\left\{x_{n}\right\}$ be a sequence generated by the following algorithm:

$$
\begin{gathered}
u_{n}=S_{r_{n}}^{(\Theta, \varphi)}\left(I-r_{n} A\right) x_{n}, \\
v_{n}=P_{C}\left(I-\lambda_{N, n} B_{N}\right) \\
\times P_{C}\left(I-\lambda_{N-1, n} B_{N-1}\right) \cdots P_{C}\left(I-\lambda_{1, n} B_{1}\right) u_{n}, \\
z_{n}=\beta_{n} x_{n}+\sigma_{n} G v_{n}+\left[\left(1-\beta_{n}\right) I-\sigma_{n} V\right] W_{n} G v_{n}, \\
k_{n}=\delta_{n} z_{n}+\left(1-\delta_{n}\right) S^{n} z_{n}, \\
x_{n+1}=\left(1-\alpha_{n}\right) x_{n}+\alpha_{n} k_{n}, \quad \forall n \geq 1,
\end{gathered}
$$

where $\nu_{k} \in\left(0,2 \zeta_{k}\right), k \in\{1,2\}$, and $\left\{\lambda_{i, n}\right\} \subset\left[a_{i}, b_{i}\right] \subset$ $\left(0,2 \eta_{i}\right), \forall i \in\{1,2, \ldots, N\}$. Assume that the conditions (i)-(iii) are satisfied. Then $\left\{x_{n}\right\}$ converges weakly to $x^{*}=\lim _{n \rightarrow \infty} P_{\Omega} x_{n}$ provided that $S_{r}^{(\Theta, \varphi)}$ is firmly nonexpansive.

Proof. As $\lim _{n \rightarrow \infty} \sigma_{n}=0,0<\liminf _{n \rightarrow \infty} \beta_{n} \leq$ $\lim \sup _{n \rightarrow \infty} \beta_{n}<1$ and $0<\liminf _{n \rightarrow \infty} r_{n} \leq$ limsup $\operatorname{sum}_{n \rightarrow \infty} r_{n}<2 \zeta$, we may assume, without loss of generality, that $\left\{\beta_{n}\right\} \subset[a, \widehat{a}] \subset[0,1],\left\{r_{n}\right\} \subset[c, \widehat{c}] \subset(0,2 \zeta)$, and $\beta_{n}+\sigma_{n}\|V\| \leq 1$ for all $n \geq 1$. First, let us show that $\lim _{n \rightarrow \infty}\left\|x_{n}-p\right\|$ exists for any $p \in \Omega$. Put

$$
\Lambda_{n}^{i}=P_{C}\left(I-\lambda_{i, n} B_{i}\right) P_{C}\left(I-\lambda_{i-1, n} B_{i-1}\right) \cdots P_{C}\left(I-\lambda_{1, n} B_{1}\right)
$$

for all $i \in\{1,2, \ldots, N\}, n \geq 1$, and $\Lambda_{n}^{0}=I$, where $I$ is the identity mapping on $H$. Then we get $v_{n}=\Lambda_{n}^{N} u_{n}$. Take $p \in \Omega$ arbitrarily. Repeating the same arguments as in the proof of Theorem 23, we can obtain that

$$
\begin{gathered}
\left\|\left(1-\beta_{n}\right) I-\sigma_{n} V\right\| \leq 1-\beta_{n}-\sigma_{n} \bar{\gamma} \\
\left\|u_{n}-p\right\|^{2} \leq\left\|x_{n}-p\right\|^{2}+r_{n}\left(r_{n}-2 \zeta\right)\left\|A x_{n}-A p\right\|^{2} \\
\leq\left\|x_{n}-p\right\|^{2} \\
\left\|v_{n}-p\right\| \leq\left\|u_{n}-p\right\| \\
\left\|G v_{n}-p\right\|^{2} \\
\leq\left\|T_{v_{2}}^{\Theta_{2}}\left(I-v_{2} A_{2}\right) v_{n}-T_{v_{2}}^{\Theta_{2}}\left(I-v_{2} A_{2}\right) p\right\|^{2} \\
+v_{1}\left(v_{1}-2 \zeta_{1}\right) \\
\times\left\|A_{1} T_{v_{2}}^{\Theta_{2}}\left(I-v_{2} A_{2}\right) v_{n}-A_{1} T_{v_{2}}^{\Theta_{2}}\left(I-v_{2} A_{2}\right) p\right\|^{2} \\
\leq\left\|T_{v_{2}}^{\Theta_{2}}\left(I-v_{2} A_{2}\right) v_{n}-T_{v_{2}}^{\Theta_{2}}\left(I-v_{2} A_{2}\right) p\right\|^{2} \\
\leq\left\|v_{n}-p\right\|^{2}+v_{2}\left(v_{2}-2 \zeta_{2}\right)\left\|A_{2} v_{n}-A_{2} p\right\|^{2} \\
\leq\left\|v_{n}-p\right\|^{2},
\end{gathered}
$$

$$
\begin{aligned}
\left\|\Lambda_{n}^{i} u_{n}-p\right\|^{2} \leq & \left\|x_{n}-p\right\|^{2}+\lambda_{i, n}\left(\lambda_{i, n}-2 \eta_{i}\right) \\
& \times\left\|B_{i} \Lambda_{n}^{i-1} u_{n}-B_{i} p\right\|^{2}, \quad i \in\{1,2, \ldots, N\},
\end{aligned}
$$

$$
\begin{aligned}
& \left\|k_{n}-p\right\|^{2} \\
& \leq\left(1+\gamma_{n}\right)\left\|z_{n}-p\right\|^{2}+\left(1-\delta_{n}\right) \\
& \quad \times\left(k-\delta_{n}\right)\left\|z_{n}-S^{n} z_{n}\right\|^{2}+c_{n} \\
& \leq\left(1+\gamma_{n}\right)\left\|z_{n}-p\right\|^{2}+c_{n} \\
& \leq\left(1+\gamma_{n}\right)\left(\left\|x_{n}-p\right\|^{2}+\sigma_{n} \frac{\|(I-V) p\|^{2}}{\bar{\gamma}-1}\right)+c_{n} \\
& \quad+2 \lambda_{i, n}\left\|\Lambda_{n}^{i-1} u_{n}-\Lambda_{n}^{i} u_{n}\right\|_{n}-p\left\|^{2} \leq\right\| x_{n}-p\left\|^{2}-\right\| \Lambda_{n}^{i-1} u_{n}-\Lambda_{n}^{i} u_{n} \|^{2} \\
& \quad \times\left\|B_{i} \Lambda_{n}^{i-1} u_{n}-B_{i} p\right\|, \quad i \in\{1,2, \ldots, N\}
\end{aligned}
$$


Utilizing (145) and (152), we obtain

$$
\begin{aligned}
&\left\|x_{n+1}-p\right\|^{2} \\
& \leq\left(1-\alpha_{n}\right)\left\|x_{n}-p\right\|^{2}+\alpha_{n}\left\|k_{n}-p\right\|^{2} \\
& \leq\left(1-\alpha_{n}\right)\left\|x_{n}-p\right\|^{2} \\
&+\alpha_{n}\left[\left(1+\gamma_{n}\right)\left(\left\|x_{n}-p\right\|^{2}+\sigma_{n} \frac{\|(I-V) p\|^{2}}{\bar{\gamma}-1}\right)+c_{n}\right] \\
& \leq\left(1+\gamma_{n}\right)\left(\left\|x_{n}-p\right\|^{2}+\sigma_{n} \frac{\|(I-V) p\|^{2}}{\bar{\gamma}-1}\right)+c_{n} \\
&=\left\|x_{n}-p\right\|^{2}+\gamma_{n}\left\|x_{n}-p\right\|^{2} \\
&+\sigma_{n}\left(1+\gamma_{n}\right) \frac{\|(I-V) p\|^{2}}{\bar{\gamma}-1}+c_{n} .
\end{aligned}
$$

Since $\sum_{n=1}^{\infty} \sigma_{n}<\infty, \sum_{n=1}^{\infty} \gamma_{n}<\infty$, and $\sum_{n=1}^{\infty} c_{n}<\infty$, by Lemma 21 we have that $\lim _{n \rightarrow \infty}\left\|x_{n}-p\right\|$ exists. Thus $\left\{x_{n}\right\}$ is bounded and so are the sequences $\left\{u_{n}\right\},\left\{v_{n}\right\},\left\{z_{n}\right\}$, and $\left\{k_{n}\right\}$.

Also, utilizing Lemmas 8 and 9(b), we obtain from (145), (148), (149), and (152) that

$$
\begin{aligned}
\| z_{n}- & p \|^{2} \\
= & \| \beta_{n}\left(x_{n}-p\right)+\left(1-\beta_{n}\right)\left(W_{n} G v_{n}-p\right) \\
& +\sigma_{n}\left(G v_{n}-V W_{n} G v_{n}\right) \|^{2} \\
\leq & \left\|\beta_{n}\left(x_{n}-p\right)+\left(1-\beta_{n}\right)\left(W_{n} G v_{n}-p\right)\right\|^{2} \\
& +2 \sigma_{n}\left\langle G v_{n}-V W_{n} G v_{n}, z_{n}-p\right\rangle \\
= & \beta_{n}\left\|x_{n}-p\right\|^{2}+\left(1-\beta_{n}\right)\left\|W_{n} G v_{n}-p\right\|^{2} \\
& -\beta_{n}\left(1-\beta_{n}\right)\left\|x_{n}-W_{n} G v_{n}\right\|^{2} \\
& +2 \sigma_{n}\left\|G v_{n}-V W_{n} G v_{n}\right\|\left\|z_{n}-p\right\| \\
= & \left\|x_{n}-p\right\|^{2}-\beta_{n}\left(1-\beta_{n}\right)\left\|x_{n}-W_{n} G v_{n}\right\|^{2} \\
& +2 \sigma_{n}\left\|G v_{n}-V W_{n} G v_{n}\right\|\left\|z_{n}-p\right\| \\
\leq & \left\|x_{n}-p\right\|^{2}+2 \sigma_{n}\left\|G v_{n}-V W_{n} G v_{n}\right\|\left\|z_{n}-p\right\|,
\end{aligned}
$$

and hence

$$
\begin{aligned}
& \left\|x_{n+1}-p\right\|^{2} \\
& \leq\left(1-\alpha_{n}\right)\left\|x_{n}-p\right\|^{2}+\alpha_{n}\left\|k_{n}-p\right\|^{2} \\
& \leq\left(1-\alpha_{n}\right)\left\|x_{n}-p\right\|^{2} \\
& \quad+\alpha_{n}\left[\left(1+\gamma_{n}\right)\left\|z_{n}-p\right\|^{2}+c_{n}\right]
\end{aligned}
$$

$$
\begin{aligned}
& \leq\left(1-\alpha_{n}\right)\left\|x_{n}-p\right\|^{2} \\
& +\alpha_{n}\left[\left(1+\gamma_{n}\right)\right. \\
& \times\left(\left\|x_{n}-p\right\|^{2}\right. \\
& -\beta_{n}\left(1-\beta_{n}\right)\left\|x_{n}-W_{n} G v_{n}\right\|^{2} \\
& +2 \sigma_{n}\left\|G v_{n}-V W_{n} G v_{n}\right\| \\
& \left.\left.\quad \times\left\|z_{n}-p\right\|\right)+c_{n}\right] \\
& \leq\left(1+\gamma_{n}\right)\left\|x_{n}-p\right\|^{2}-\alpha_{n}\left(1+\gamma_{n}\right) \beta_{n} \\
& \times\left(1-\beta_{n}\right)\left\|x_{n}-W_{n} G v_{n}\right\|^{2} \\
& +2 \sigma_{n}\left(1+\gamma_{n}\right)\left\|G v_{n}-V W_{n} G v_{n}\right\|\left\|z_{n}-p\right\|+c_{n} .
\end{aligned}
$$

So, it follows that

$$
\begin{aligned}
\alpha(1 & \left.+\gamma_{n}\right) a(1-\widehat{a})\left\|x_{n}-W_{n} G v_{n}\right\|^{2} \\
\leq & \alpha_{n}\left(1+\gamma_{n}\right) \beta_{n}\left(1-\beta_{n}\right)\left\|x_{n}-W_{n} G v_{n}\right\|^{2} \\
\leq & \left\|x_{n}-p\right\|^{2}-\left\|x_{n+1}-p\right\|^{2}+\gamma_{n}\left\|x_{n}-p\right\|^{2} \\
& +2 \sigma_{n}\left(1+\gamma_{n}\right)\left\|G v_{n}-V W_{n} G v_{n}\right\|\left\|z_{n}-p\right\|+c_{n} .
\end{aligned}
$$

Since $\lim _{n \rightarrow \infty} \sigma_{n}=0, \lim _{n \rightarrow \infty} \gamma_{n}=0$, and $\lim _{n \rightarrow \infty} c_{n}=0$, it follows from the existence of $\lim _{n \rightarrow \infty}\left\|x_{n}-p\right\|$ and the boundedness of $\left\{x_{n}\right\},\left\{z_{n}\right\}$, and $\left\{v_{n}\right\}$ that

$$
\lim _{n \rightarrow \infty}\left\|x_{n}-W_{n} G v_{n}\right\|=0 .
$$

Note that

$$
\begin{aligned}
& \left\|z_{n}-x_{n}\right\| \\
& \quad=\left\|\left(1-\beta_{n}\right)\left(W_{n} G v_{n}-x_{n}\right)+\sigma_{n}\left(G v_{n}-V W_{n} G v_{n}\right)\right\| \\
& \quad \leq\left\|W_{n} G v_{n}-x_{n}\right\|+\sigma_{n}\left\|G v_{n}-V W_{n} G v_{n}\right\| .
\end{aligned}
$$

Hence, it follows from (158) and $\lim _{n \rightarrow \infty} \sigma_{n}=0$ that

$$
\lim _{n \rightarrow \infty}\left\|x_{n}-z_{n}\right\|=0 \text {. }
$$

In the meantime, from (152) and (155) it follows that

$$
\begin{aligned}
& \left\|x_{n+1}-p\right\|^{2} \\
& \leq\left(1-\alpha_{n}\right)\left\|x_{n}-p\right\|^{2}+\alpha_{n}\left\|k_{n}-p\right\|^{2} \\
& \leq\left(1-\alpha_{n}\right)\left\|x_{n}-p\right\|^{2} \\
& \quad+\alpha_{n}\left[\left(1+\gamma_{n}\right)\left\|z_{n}-p\right\|^{2}+\left(1-\delta_{n}\right)\right. \\
& \left.\quad \times\left(k-\delta_{n}\right)\left\|z_{n}-S^{n} z_{n}\right\|^{2}+c_{n}\right]
\end{aligned}
$$




$$
\begin{aligned}
& \leq\left(1-\alpha_{n}\right)\left\|x_{n}-p\right\|^{2} \\
& +\alpha_{n}\left[\left(1+\gamma_{n}\right)\right. \\
& \times\left(\left\|x_{n}-p\right\|^{2}\right. \\
& \left.+2 \sigma_{n}\left\|G v_{n}-V W_{n} G v_{n}\right\|\left\|z_{n}-p\right\|\right) \\
& \left.+\left(1-\delta_{n}\right)\left(k-\delta_{n}\right)\left\|z_{n}-S^{n} z_{n}\right\|^{2}+c_{n}\right] \\
& \leq\left(1-\alpha_{n}\right)\left\|x_{n}-p\right\|^{2}+\alpha_{n}\left(1+\gamma_{n}\right) \\
& \times\left(\left\|x_{n}-p\right\|^{2}+2 \sigma_{n}\left\|G v_{n}-V W_{n} G v_{n}\right\|\left\|z_{n}-p\right\|\right) \\
& +\alpha_{n}\left(1-\delta_{n}\right)\left(k-\delta_{n}\right)\left\|z_{n}-S^{n} z_{n}\right\|^{2}+c_{n} \\
& \leq\left(1-\alpha_{n}\right)\left\|x_{n}-p\right\|^{2}+\alpha_{n}\left(1+\gamma_{n}\right)\left\|x_{n}-p\right\|^{2} \\
& +2\left(1+\gamma_{n}\right) \sigma_{n}\left\|G v_{n}-V W_{n} G v_{n}\right\|\left\|z_{n}-p\right\| \\
& +\alpha_{n}\left(1-\delta_{n}\right)\left(k-\delta_{n}\right)\left\|z_{n}-S^{n} z_{n}\right\|^{2}+c_{n} \\
& \leq\left(1+\gamma_{n}\right)\left\|x_{n}-p\right\|^{2}+2 \sigma_{n}\left(1+\gamma_{n}\right) \\
& \times\left\|G v_{n}-V W_{n} G v_{n}\right\|\left\|z_{n}-p\right\| \\
& +\alpha_{n}\left(1-\delta_{n}\right)\left(k-\delta_{n}\right)\left\|z_{n}-S^{n} z_{n}\right\|^{2}+c_{n},
\end{aligned}
$$

which, together with $0<k+\epsilon \leq \delta_{n} \leq d<1$, leads to

$$
\begin{aligned}
& \alpha(1-d) \epsilon\left\|z_{n}-S^{n} z_{n}\right\|^{2} \\
& \leq \alpha_{n}\left(1-\delta_{n}\right)\left(\delta_{n}-k\right)\left\|z_{n}-S^{n} z_{n}\right\|^{2} \\
& \leq\left\|x_{n}-p\right\|^{2}-\left\|x_{n+1}-p\right\|^{2}+\gamma_{n}\left\|x_{n}-p\right\|^{2} \\
&+2 \sigma_{n}\left(1+\gamma_{n}\right)\left\|G v_{n}-V W_{n} G v_{n}\right\|\left\|z_{n}-p\right\|+c_{n} .
\end{aligned}
$$

Consequently, from $\lim _{n \rightarrow \infty} \sigma_{n}=0, \lim _{n \rightarrow \infty} \gamma_{n}=0$, $\lim _{n \rightarrow \infty} c_{n}=0$, and the existence of $\lim _{n \rightarrow \infty}\left\|x_{n}-p\right\|$, we get

$$
\lim _{n \rightarrow \infty}\left\|z_{n}-S^{n} z_{n}\right\|=0 .
$$

Since $k_{n}-z_{n}=\left(1-\delta_{n}\right)\left(S^{n} z_{n}-z_{n}\right)$, from (163) we have

$$
\lim _{n \rightarrow \infty}\left\|k_{n}-z_{n}\right\|=0 \text {. }
$$

Note that

$$
\left\|x_{n+1}-x_{n}\right\|=\alpha_{n}\left\|k_{n}-x_{n}\right\| \leq\left\|k_{n}-z_{n}\right\|+\left\|z_{n}-x_{n}\right\| .
$$

Hence from (160) and (164) we have

$$
\lim _{n \rightarrow \infty}\left\|x_{n+1}-x_{n}\right\|=0 \text {. }
$$

Repeating the same arguments as those of Step 3 in the proof of Theorem 23, we can obtain that $\left\|x_{n}-u_{n}\right\| \rightarrow 0,\left\|x_{n}-v_{n}\right\| \rightarrow$ $0,\left\|v_{n}-G v_{n}\right\| \rightarrow 0,\left\|x_{n}-W x_{n}\right\| \rightarrow 0,\left\|z_{n}-S z_{n}\right\| \rightarrow 0$, and $\left\|\Lambda_{n}^{i-1} u_{n}-\Lambda_{n}^{i} u_{n}\right\| \rightarrow 0, i \in\{1,2, \ldots, N\}$ as $n \rightarrow \infty$.
Since $\left\{x_{n}\right\}$ is bounded, there exists a subsequence $\left\{x_{n_{i}}\right\}$ of $\left\{x_{n}\right\}$ which converges weakly to $w$. It is easy to see that $v_{n_{i}} \rightarrow w, u_{n_{i}} \rightarrow w, \Lambda_{n_{i}}^{m} u_{n_{i}} \rightarrow w$, and $z_{n_{i}} \rightarrow w$, where $m \in\{1,2, \ldots, N\}$. Since $S$ is uniformly continuous and $\| z_{n}-$ $S z_{n} \| \rightarrow 0$ as $n \rightarrow \infty$, we get $\lim _{n \rightarrow \infty}\left\|z_{n}-S^{m} z_{n}\right\|=0$ for any $m \geq 1$. Hence from Lemma 17, we obtain $w \in \operatorname{Fix}(S)$. In the meantime, utilizing Lemma 12, we deduce from $v_{n_{i}} \rightarrow$ $w, x_{n_{i}} \rightarrow w,\left\|v_{n}-G v_{n}\right\| \rightarrow 0$, and $\left\|x_{n}-W x_{n}\right\| \rightarrow 0$ that $w \in$ $\operatorname{SGEP}(G)$ and $w \in \operatorname{Fix}(W)=\cap_{n=1}^{\infty} \operatorname{Fix}\left(T_{n}\right)$ (due to Lemma 11). Repeating the same arguments as those of Step 4 in the proof of Theorem 23, we can conclude that $w \in \bigcap_{m=1}^{N} \operatorname{VI}\left(C, B_{m}\right)$ and $w \in \operatorname{GMEP}(\Theta, \varphi, A)$. Consequently, $w \in \Omega$. This shows that $\omega_{w}\left(x_{n}\right) \subset \Omega$.

Next let us show that $\omega_{w}\left(x_{n}\right)$ is a single-point set. As a matter of fact, let $\left\{x_{n_{j}}\right\}$ be another subsequence of $\left\{x_{n}\right\}$ such that $x_{n_{j}} \rightarrow w^{\prime}$. Then we get $w^{\prime} \in \Omega$. If $w \neq w^{\prime}$, from the Opial condition, we have

$$
\begin{aligned}
& \lim _{n \rightarrow \infty}\left\|x_{n}-w\right\| \\
& =\lim _{i \rightarrow \infty}\left\|x_{n_{i}}-w\right\|<\lim _{i \rightarrow \infty}\left\|x_{n_{i}}-w^{\prime}\right\| \\
& =\lim _{n \rightarrow \infty}\left\|x_{n}-w^{\prime}\right\|=\lim _{j \rightarrow \infty}\left\|x_{n_{j}}-w^{\prime}\right\| \\
& <\lim _{j \rightarrow \infty}\left\|x_{n_{j}}-w\right\|=\lim _{n \rightarrow \infty}\left\|x_{n}-w\right\| .
\end{aligned}
$$

This attains a contradiction. So we have $w=w^{\prime}$. Put $w_{n}=$ $P_{\Omega} x_{n}$. Since $w \in \Omega$, we have $\left\langle x_{n}-w_{n}, w_{n}-w\right\rangle \geq 0$. By Lemma 21 , we have that $\left\{w_{n}\right\}$ converges strongly to some $\widetilde{w} \in$ $\Omega$. Since $\left\{x_{n}\right\}$ converges weakly to $w$, we have

$$
\langle w-\widetilde{w}, \widetilde{w}-w\rangle \geq 0
$$

Therefore we obtain $w=\widetilde{w}=\lim _{n \rightarrow \infty} P_{\Omega} x_{n}$. This completes the proof.

Corollary 29. Choose $N=2$ in Theorem 28. For any $x_{1} \in H$ the iterative scheme (145) reduces to the following iterative one:

$$
\begin{gathered}
u_{n}=S_{r_{n}}^{(\Theta, \varphi)}\left(I-r_{n} A\right) x_{n}, \\
v_{n}=P_{C}\left(I-\lambda_{2, n} B_{2}\right) P_{C}\left(I-\lambda_{1, n} B_{1}\right) u_{n}, \\
z_{n}=\beta_{n} x_{n}+\left(1-\beta_{n}\right) G v_{n}+\sigma_{n}(I-V) G v_{n}, \\
k_{n}=\delta_{n} z_{n}+\left(1-\delta_{n}\right) S^{n} z_{n}, \\
x_{n+1}=\left(1-\alpha_{n}\right) x_{n}+\alpha_{n} k_{n}, \quad \forall n \geq 1,
\end{gathered}
$$

where $\nu_{k} \in\left(0,2 \zeta_{k}\right)$ and $\left\{\lambda_{i, n}\right\} \subset\left[a_{i}, b_{i}\right] \subset\left(0,2 \eta_{i}\right)$ for $k=1,2$ and $i=1,2$. Then $\left\{x_{n}\right\}$ converges weakly to $x^{*}=\lim _{n \rightarrow \infty} P_{\Omega} x_{n}$ provided that $S_{r}^{(\Theta, \varphi)}$ is firmly nonexpansive. 
Corollary 30. Choose $N=1$ and $T_{n} \equiv I$ the identity operator of $H$ in Theorem 28. For any $x_{1} \in H$ the iterative scheme (145) reduces to the following iterative one:

$$
\begin{gathered}
u_{n}=S_{r_{n}}^{(\Theta, \varphi)}\left(I-r_{n} A\right) x_{n}, \\
v_{n}=P_{C}\left(I-\lambda_{1, n} B_{1}\right) u_{n}, \\
z_{n}=\beta_{n} x_{n}+\sigma_{n} G v_{n}+\left[\left(1-\beta_{n}\right) I-\sigma_{n} V\right] G v_{n}, \\
k_{n}=\delta_{n} z_{n}+\left(1-\delta_{n}\right) S^{n} z_{n}, \\
x_{n+1}=\left(1-\alpha_{n}\right) x_{n}+\alpha_{n} k_{n}, \quad \forall n \geq 1,
\end{gathered}
$$

where $\nu_{k} \in\left(0,2 \zeta_{k}\right)$ and $\left\{\lambda_{1, n}\right\} \subset\left[a_{1}, b_{1}\right] \subset\left(0,2 \eta_{1}\right)$ for $k=1,2$. Then $\left\{x_{n}\right\}$ converges weakly to $x^{*}=\lim _{n \rightarrow \infty} P_{\Omega} x_{n}$ provided that $S_{r}^{(\Theta, \varphi)}$ is firmly nonexpansive.

In the following, we provide a numerical example to illustrate how Corollary 30 works.

Example 31. Let $H=\mathbf{R}^{2}$ with inner product $\langle\cdot, \cdot\rangle$ and norm $\|\cdot\|$ which are defined by

$$
\langle x, y\rangle=a c+b d, \quad\|x\|=\sqrt{a^{2}+b^{2}},
$$

for all $x, y \in \mathbf{R}^{2}$ with $x=(a, b)$ and $y=(c, d)$. Let $C=\{(a, a)$ : $a \in \mathbf{R}\}$. Clearly, $C$ is a nonempty closed convex subset of a real Hilbert space $H=\mathbf{R}^{2}$. Let $K(x)=(1 / 2)\|x\|^{2}, \forall x \in H$, $\Theta(x, y)=\Theta_{1}(x, y)=\Theta_{2}(x, y)=0, \forall(x, y) \in C \times C$, and $\varphi=0, \forall x \in C$. Then $\Theta, \Theta_{1}$, and $\Theta_{2}$ are three bifunctions from $C \times C$ to $\mathbf{R}$ satisfying $(\mathrm{H} 1)-(\mathrm{H} 4)$ and $\varphi: C \rightarrow \mathbf{R}$ is a lower semicontinuous and convex functional. Let $V$ be a $\bar{\gamma}$-strongly positive bounded linear operator with $\bar{\gamma} \in(1,2]$, let $A, A_{k}: H \rightarrow H$ and $B_{1}: C \rightarrow H$ be $\zeta$-inverse strongly monotone, $\zeta_{k}$-inverse strongly monotone, and $\eta_{1}$ inverse strongly monotone, respectively, for $k=1,2$, and let $S: C \rightarrow C$ be a uniformly continuous asymptotically $k$ strict pseudocontractive mapping in the intermediate sense for some $0 \leq k<1$ with sequence $\left\{\gamma_{n}\right\} \subset[0, \infty)$ such that $\sum_{n=1}^{\infty} \gamma_{n}<\infty$ and $\left\{c_{n}\right\} \subset[0, \infty)$ such that $\sum_{n=1}^{\infty} c_{n}<\infty$ such that $\Omega:=\operatorname{GMEP}(\Theta, \varphi, A) \cap \operatorname{SGEP}(G) \cap \operatorname{VI}(C, B) \cap \operatorname{Fix}(S)$ is nonempty, for instance, putting

$$
\begin{gathered}
A=\left\{\begin{array}{ll}
\frac{3}{5} & \frac{2}{5} \\
\frac{2}{5} & \frac{3}{5}
\end{array}\right\}, \quad B_{1}=S=\left\{\begin{array}{ll}
\frac{2}{3} & \frac{1}{3} \\
\frac{1}{3} & \frac{2}{3}
\end{array}\right\}, \\
V=\frac{5}{4} A, \quad A_{1}=I-A=\left\{\begin{array}{cc}
\frac{2}{5} & -\frac{2}{5} \\
-\frac{2}{5} & \frac{2}{5}
\end{array}\right\}, \\
A_{2}=I-B_{1}=\left\{\begin{array}{cc}
\frac{1}{3} & -\frac{1}{3} \\
-\frac{1}{3} & \frac{1}{3}
\end{array}\right\} .
\end{gathered}
$$

It is easy to see that $\|A\|=\left\|B_{1}\right\|=\|S\|=1$, that $A$ is $\zeta$-inverse strongly monotone with $\zeta=1 / 2$, that $V$ is a $5 / 4$-strongly positive bounded linear operator, that $B_{1}, A_{1}$, and $A_{2}$ are $1 / 2$-inverse strongly monotone, and that $S$ is a nonexpansive mapping, that is, a uniformly continuous asymptotically 0 strict pseudocontractive mapping in the intermediate sense with sequences $\left\{\gamma_{n}\right\}\left(\gamma_{n} \equiv 0\right)$ and $\left\{c_{n}\right\}\left(c_{n} \equiv 0\right)$. Moreover, it is clear that $\operatorname{Fix}(S)=C, \operatorname{GMEP}(\Theta, \varphi, A)=\{0\}, \operatorname{VI}\left(C, B_{1}\right)=$ $\{0\}$, and $\operatorname{SGEP}(G)=C$. Hence, $\Omega:=\operatorname{GMEP}(\Theta, \varphi, A) \cap$ $\operatorname{SGEP}(G) \cap \operatorname{VI}\left(C, B_{1}\right) \cap \operatorname{Fix}(S)=\{0\}$. In this case, from iterative scheme (170) in Corollary 30, we obtain that, for any given $x_{1} \in C$,

$$
\begin{aligned}
& u_{n}=S_{r_{n}}^{(\Theta, \varphi)}\left(I-r_{n} A\right) x_{n}=P_{C}\left(I-r_{n} A\right) x_{n}=\left(1-r_{n}\right) x_{n}, \\
& v_{n}=P_{C}\left(I-\lambda_{1, n} B_{1}\right) u_{n}=\left(1-\lambda_{1, n}\right) u_{n} \\
& =\left(1-\lambda_{1, n}\right)\left(1-r_{n}\right) x_{n}, \\
& z_{n}=\beta_{n} x_{n}+\left(1-\beta_{n}\right) G v_{n}+\sigma_{n}(I-V) G v_{n} \\
& =\beta_{n} x_{n}+\left(1-\beta_{n}\right) T_{v_{1}}^{\Theta_{1}}\left(I-v_{1} A_{1}\right) T_{v_{2}}^{\Theta_{2}}\left(I-v_{2} A_{2}\right) v_{n} \\
& +\sigma_{n}(I-V) T_{v_{1}}^{\Theta_{1}}\left(I-v_{1} A_{1}\right) T_{v_{2}}^{\Theta_{2}}\left(I-v_{2} A_{2}\right) v_{n} \\
& =\beta_{n} x_{n}+\left(1-\beta_{n}\right) v_{n}+\sigma_{n}\left(I-\frac{5}{4} A\right) v_{n} \\
& =\beta_{n} x_{n}+\left(1-\beta_{n}\right) v_{n}-\frac{1}{4} \sigma_{n} v_{n} \\
& =\beta_{n} x_{n}+\left(1-\beta_{n}-\frac{1}{4} \sigma_{n}\right) v_{n} \\
& =\beta_{n} x_{n}+\left(1-\beta_{n}-\frac{1}{4} \sigma_{n}\right)\left(1-\lambda_{1, n}\right)\left(1-r_{n}\right) x_{n} \\
& =\left[\beta_{n}+\left(1-\beta_{n}-\frac{1}{4} \sigma_{n}\right)\left(1-\lambda_{1, n}\right)\left(1-r_{n}\right)\right] x_{n}, \\
& k_{n}=\delta_{n} z_{n}+\left(1-\delta_{n}\right) S^{n} z_{n}=\delta_{n} z_{n}+\left(1-\delta_{n}\right) z_{n}=z_{n} \\
& =\left[\beta_{n}+\left(1-\beta_{n}-\frac{1}{4} \sigma_{n}\right)\left(1-\lambda_{1, n}\right)\left(1-r_{n}\right)\right] x_{n}, \\
& x_{n+1}=\left(1-\alpha_{n}\right) x_{n}+\alpha_{n} k_{n} \\
& =\left(1-\alpha_{n}\right) x_{n} \\
& +\alpha_{n}\left[\beta_{n}+\left(1-\beta_{n}-\frac{1}{4} \sigma_{n}\right)\left(1-\lambda_{1, n}\right)\left(1-r_{n}\right)\right] x_{n} \\
& =\left\{1-\alpha_{n}\right. \\
& \left.+\alpha_{n}\left[\beta_{n}+\left(1-\beta_{n}-\frac{1}{4} \sigma_{n}\right)\left(1-\lambda_{1, n}\right)\left(1-r_{n}\right)\right]\right\} x_{n} .
\end{aligned}
$$

Whenever $0<\alpha \leq \alpha_{n} \leq 1,\left\{\lambda_{1, n}\right\} \subset\left[a_{1}, b_{1}\right] \subset(0,1),\left\{\beta_{n}\right\} \subset$ $[c, \widehat{c}] \subset(0,1),\left\{r_{n}\right\} \subset[e, \hat{e}] \subset(0,1)$ and $\left\{\sigma_{n}\right\} \subset[0,1]$ satisfying $(1 / 4) \sigma_{n} \leq 1-\widehat{c}$, we have

$$
\begin{aligned}
& \left\|x_{n+1}\right\| \\
& \quad=\left\{1-\alpha_{n}+\alpha_{n}\left[\beta_{n}+\left(1-\beta_{n}-\frac{1}{4} \sigma_{n}\right)\right.\right.
\end{aligned}
$$




$$
\begin{aligned}
& \left.\left.\times\left(1-\lambda_{1, n}\right)\left(1-r_{n}\right)\right]\right\}\left\|x_{n}\right\| \\
& \leq\left\{1-\alpha_{n}+\alpha_{n}\left[\beta_{n}+\left(1-\beta_{n}\right)\right.\right. \\
& \left.\left.\times\left(1-\lambda_{1, n}\right)\left(1-r_{n}\right)\right]\right\}\left\|x_{n}\right\| \\
& \leq\left\{1-\alpha_{n}+\alpha_{n}\left[\beta_{n}+\left(1-\beta_{n}\right)\right.\right. \\
& \left.\left.\times\left(1-a_{1}\right)(1-e)\right]\right\}\left\|x_{n}\right\| \\
& =\left[1-\alpha_{n}\left(1-\beta_{n}\right)+\alpha_{n}\left(1-\beta_{n}\right)\right. \\
& \left.\times\left(1-a_{1}\right)(1-e)\right]\left\|x_{n}\right\| \\
& =\left[1-\alpha_{n}\left(1-\beta_{n}\right)\left(1-\left(1-a_{1}\right)(1-e)\right)\right]\left\|x_{n}\right\| \\
& \leq\left[1-\alpha(1-\widehat{c})\left(1-\left(1-a_{1}\right)(1-e)\right)\right]\left\|x_{n}\right\| \\
& \leq\left[1-\alpha(1-\widehat{c})\left(1-\left(1-a_{1}\right)(1-e)\right)\right]^{n}\left\|x_{1}\right\| .
\end{aligned}
$$

Since $0<\alpha(1-\widehat{c})\left(1-\left(1-a_{1}\right)(1-e)\right)<1$, we immediately get

$$
\lim _{n \rightarrow \infty}\left\|x_{n}\right\|=0 \text {. }
$$

This shows that $\left\{x_{n}\right\}$ converges to the unique element 0 of $\Omega$.

\section{Conflict of Interests}

The authors declare that there is no conflict of interests regarding the publication of this paper.

\section{Acknowledgments}

This research was partially supported by the National Science Foundation of China (11071169), the Innovation Program of Shanghai Municipal Education Commission (09ZZ133), and the Ph.D. Program Foundation of the Ministry of Education of China (20123127110002). This work was supported partly by the National Science Council of Taiwan.

\section{References}

[1] J. L. Lions, Quelques Méthodes de Résolution des Problèmes aux Limites Non Linéaires, Dunod, Paris, 1969.

[2] R. Glowinski, Numerical Methods for Nonlinear Variational Problems, Springer, New York, NY, USA, 1984.

[3] W. Takahashi, Nonlinear Functional Analysis, Yokohama Publishers, Yokohama, Japan, 2000.

[4] J. T. Oden, Quantitative Methods on Nonlinear Mechanics, Prentice-Hall, Englewood Cliffs, NJ, USA, 1986.

[5] E. Zeidler, Nonlinear Functional Analysis and Its Applications, Springer, New York, NY, USA, 1985.

[6] L. C. Zeng, "Iterative algorithms for finding approximate solutions for general strongly nonlinear variational inequalities," Journal of Mathematical Analysis and Applications, vol. 187, no. 2, pp. 352-360, 1994.
[7] L. C. Zeng, "Iterative algorithm for finding approximate solutions to completely generalized strongly nonlinear quasivariational inequalities," Journal of Mathematical Analysis and Applications, vol. 201, no. 1, pp. 180-194, 1996.

[8] Y. Yao and J. C. Yao, "On modified iterative method for nonexpansive mappings and monotone mappings," Applied Mathematics and Computation, vol. 186, no. 2, pp. 1551-1558, 2007.

[9] L. C. Ceng, Q. H. Ansari, and J. C. Yao, "Iterative methods for triple hierarchical variational inequalities in Hilbert spaces," Journal of Optimization Theory and Applications, vol. 151, no. 3, pp. 489-512, 2011.

[10] L. C. Ceng, M. Teboulle, and J. C. Yao, "Weak convergence of an iterative method for pseudomonotone variational inequalities and fixed-point problems," Journal of Optimization Theory and Applications, vol. 146, no. 1, pp. 19-31, 2010.

[11] L. C. Ceng, Q. H. Ansari, M. M. Wong, and J. C. Yao, "Mann type hybrid extragradient method for variational inequalities, variational inclusions and fixed point problems," Fixed Point Theory, vol. 13, no. 2, pp. 403-422, 2012.

[12] G. M. Korpelevič, "An extragradient method for finding saddle points and for other problems," Ékonomika i Matematicheskie Metody, vol. 12, no. 4, pp. 747-756, 1976.

[13] L. C. Ceng, C. Y. Wang, and J. C. Yao, "Strong convergence theorems by a relaxed extragradient method for a general system of variational inequalities," Mathematical Methods of Operations Research, vol. 67, no. 3, pp. 375-390, 2008.

[14] L. C. Zeng and J. C. Yao, "Strong convergence theorem by an extragradient method for fixed point problems and variational inequality problems," Taiwanese Journal of Mathematics, vol. 10, no. 5, pp. 1293-1303, 2006.

[15] L. C. Ceng and J. C. Yao, "An extragradient-like approximation method for variational inequality problems and fixed point problems," Applied Mathematics and Computation, vol. 190, no. 1, pp. 205-215, 2007.

[16] N. Nadezhkina and W. Takahashi, "Weak convergence theorem by an extragradient method for nonexpansive mappings and monotone mappings," Journal of Optimization Theory and Applications, vol. 128, no. 1, pp. 191-201, 2006.

[17] N. Nadezhkina and W. Takahashi, "Strong convergence theorem by a hybrid method for nonexpansive mappings and Lipschitz-continuous monotone mappings," SIAM Journal on Optimization, vol. 16, no. 4, pp. 1230-1241, 2006.

[18] J. W. Peng and J. C. Yao, "A new hybrid-extragradient method for generalized mixed equilibrium problems, fixed point problems and variational inequality problems," Taiwanese Journal of Mathematics, vol. 12, no. 6, pp. 1401-1432, 2008.

[19] L. C. Ceng and J. C. Yao, "A relaxed extragradient-like method for a generalized mixed equilibrium problem, a general system of generalized equilibria and a fixed point problem," Nonlinear Analysis: Theory, Methods \& Applications, vol. 72, no. 3-4, pp. 1922-1937, 2010.

[20] L. C. Ceng, Q. H. Ansari, and S. Schaible, "Hybrid extragradient-like methods for generalized mixed equilibrium problems, systems of generalized equilibrium problems and optimization problems," Journal of Global Optimization, vol. 53, no. 1, pp. 69-96, 2012.

[21] L. C. Ceng, N. Hadjisavvas, and N. C. Wong, "Strong convergence theorem by a hybrid extragradient-like approximation method for variational inequalities and fixed point problems," Journal of Global Optimization, vol. 46, no. 4, pp. 635-646, 2010. 
[22] S. Huang, "Hybrid extragradient methods for asymptotically strict pseudo-contractions in the intermediate sense and variational inequality problems," Optimization, vol. 60, no. 6, pp. 739-754, 2011.

[23] L. C. Ceng and A. Petruşel, "Relaxed extragradient-like method for general system of generalized mixed equilibria and fixed point problem," Taiwanese Journal of Mathematics, vol. 16, no. 2, pp. 445-478, 2012.

[24] L. C. Ceng, H. Y. Hu, and M. M. Wong, "Strong and weak convergence theorems for generalized mixed equilibrium problem with perturbation and fixed pointed problem of infinitely many nonexpansive mappings," Taiwanese Journal of Mathematics, vol. 15, no. 3, pp. 1341-1367, 2011.

[25] Y. Yao, Y. C. Liou, and J. C. Yao, "New relaxed hybrid-extragradient method for fixed point problems, a general system of variational inequality problems and generalized mixed equilibrium problems," Optimization, vol. 60, no. 3, pp. 395-412, 2011.

[26] G. Cai and S. Bu, "Strong and weak convergence theorems for general mixed equilibrium problems and variational inequality problems and fixed point problems in Hilbert spaces," Journal of Computational and Applied Mathematics, vol. 247, pp. 34-52, 2013.

[27] Y. Yao, Y. J. Cho, and Y. C. Liou, "Algorithms of common solutions for variational inclusions, mixed equilibrium problems and fixed point problems," European Journal of Operational Research, vol. 212, no. 2, pp. 242-250, 2011.

[28] L. C. Ceng, S. M. Guu, and J. C. Yao, "Hybrid iterative method for finding common solutions of generalized mixed equilibrium and fixed point problems," Fixed Point Theory and Applications, vol. 2012, article 92, 19 pages, 2012.

[29] S. Takahashi and W. Takahashi, "Strong convergence theorem for a generalized equilibrium problem and a nonexpansive mapping in a Hilbert space," Nonlinear Analysis: Theory, Methods \& Applications, vol. 69, no. 3, pp. 1025-1033, 2008.

[30] Y. Yao, H. Zhou, and Y. C. Liou, "Weak and strong convergence theorems for an asymptotically $k$-strict pseudo-contraction and a mixed equilibrium problem," Journal of the Korean Mathematical Society, vol. 46, no. 3, pp. 561-576, 2009.

[31] Y. Yao, M. A. Noor, S. Zainab, and Y. C. Liou, "Mixed equilibrium problems and optimization problems," Journal of Mathematical Analysis and Applications, vol. 354, no. 1, pp. 319329, 2009.

[32] L. C. Ceng and J. C. Yao, "A hybrid iterative scheme for mixed equilibrium problems and fixed point problems," Journal of Computational and Applied Mathematics, vol. 214, no. 1, pp. 186201, 2008.

[33] L. C. Ceng and J. C. Yao, "Hybrid viscosity approximation schemes for equilibrium problems and fixed point problems of infinitely many nonexpansive mappings," Applied Mathematics and Computation, vol. 198, no. 2, pp. 729-741, 2008.

[34] L. C. Ceng, A. Petruşel, and J. C. Yao, "Iterative approaches to solving equilibrium problems and fixed point problems of infinitely many nonexpansive mappings," Journal of Optimization Theory and Applications, vol. 143, no. 1, pp. 37-58, 2009.

[35] T. H. Kim and H. K. Xu, "Convergence of the modified Mann's iteration method for asymptotically strict pseudo-contractions," Nonlinear Analysis: Theory, Methods \& Applications, vol. 68, no. 9, pp. 2828-2836, 2008.

[36] D. R. Sahu, H. K. Xu, and J. C. Yao, "Asymptotically strict pseudocontractive mappings in the intermediate sense," Nonlinear Analysis: Theory, Methods \& Applications, vol. 70, no. 10, pp. 3502-3511, 2009.
[37] J. G. O'Hara, P. Pillay, and H. K. Xu, "Iterative approaches to convex feasibility problems in Banach spaces," Nonlinear Analysis: Theory, Methods \& Applications, vol. 64, no. 9, pp. 20222042, 2006.

[38] K. Goebel and W. A. Kirk, Topics on Metric Fixed-Point Theory, Cambridge University Press, Cambridge, UK, 1990.

[39] R. T. Rockafellar, "Monotone operators and the proximal point algorithm," SIAM Journal on Control and Optimization, vol. 14, no. 5, pp. 877-898, 1976.

[40] J. Górnicki, "Weak convergence theorems for asymptotically nonexpansive mappings in uniformly convex Banach spaces," Commentationes Mathematicae Universitatis Carolinae, vol. 30, no. 2, pp. 249-252, 1989.

[41] H. K. Xu, "Existence and convergence for fixed points of mappings of asymptotically nonexpansive type," Nonlinear Analysis: Theory, Methods \& Applications, vol. 16, no. 12, pp. 1139-1146, 1991.

[42] G. Marino and H. K. Xu, "Weak and strong convergence theorems for strict pseudo-contractions in Hilbert spaces," Journal of Mathematical Analysis and Applications, vol. 329, no. 1, pp. 336-346, 2007.

[43] M. O. Osilike, S. C. Aniagbosor, and B. G. Akuchu, "Fixed points of asymptotically demicontractive mappings in arbitrary Banach spaces," Panamerican Mathematical Journal, vol. 12, no. 2, pp. 77-88, 2002.

[44] C. Martinez-Yanes and H. K. Xu, "Strong convergence of the CQ method for fixed point iteration processes," Nonlinear Analysis: Theory, Methods \& Applications, vol. 64, no. 11, pp. 2400-2411, 2006.

[45] Y. Yao, Y. C. Liou, and J. C. Yao, "Convergence theorem for equilibrium problems and fixed point problems of infinite family of nonexpansive mappings," Fixed Point Theory and Applications, vol. 2007, Article ID 64363, 12 pages, 2007. 


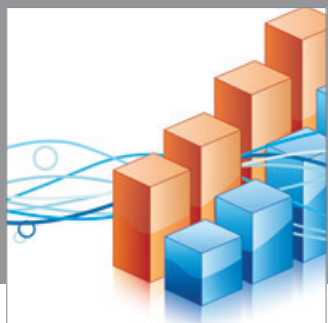

Advances in

Operations Research

mansans

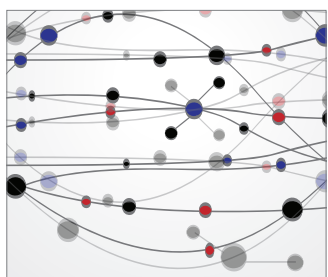

The Scientific World Journal
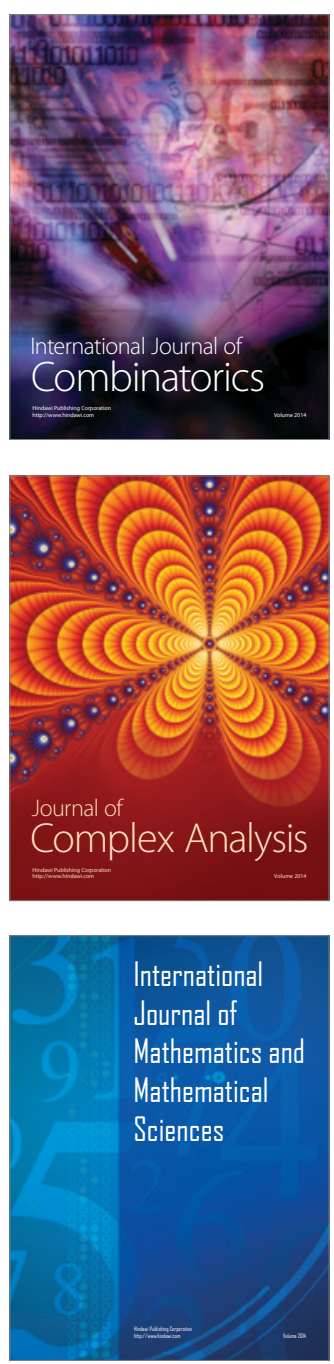
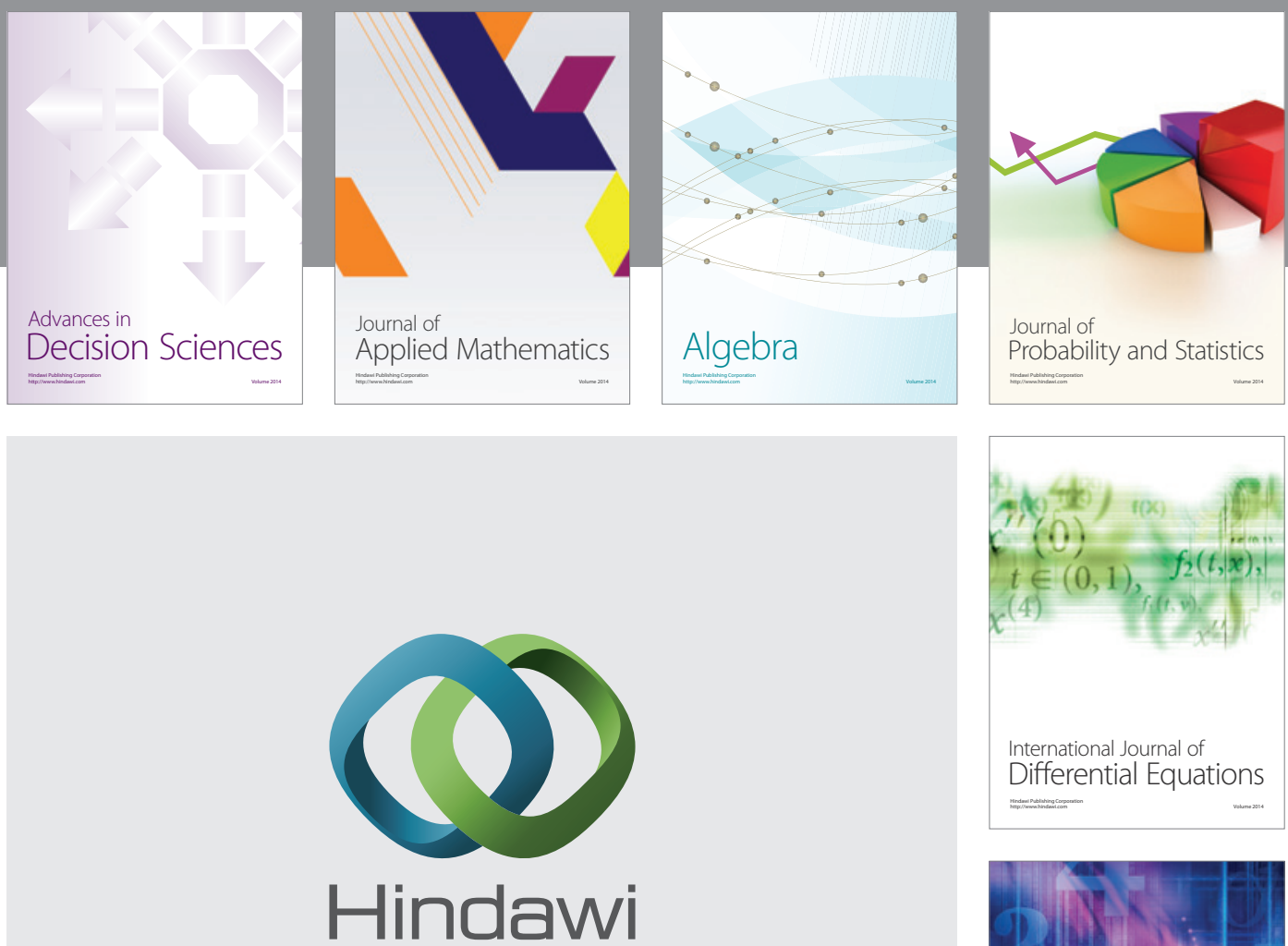

Submit your manuscripts at http://www.hindawi.com
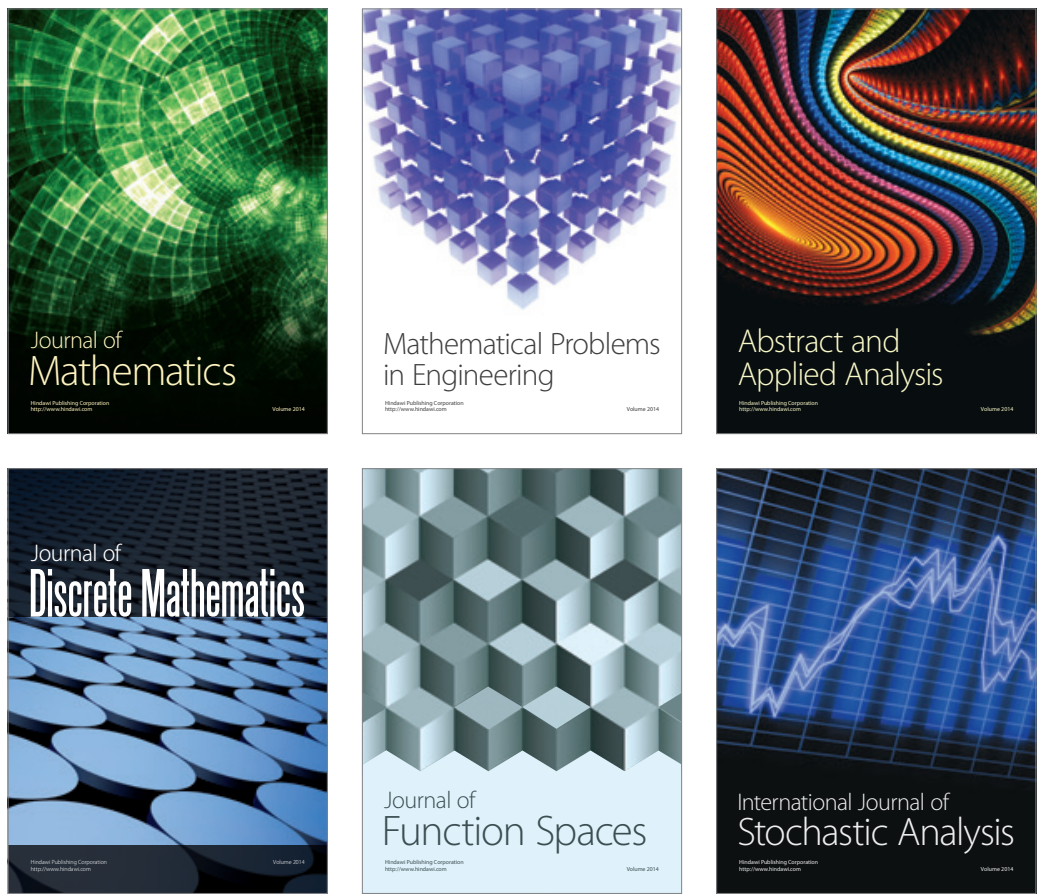

Journal of

Function Spaces

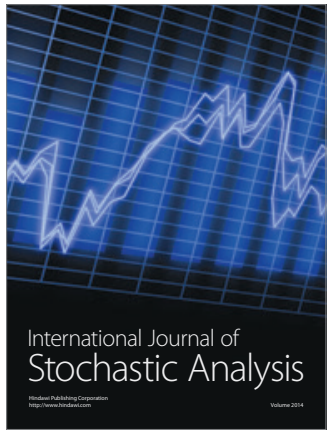

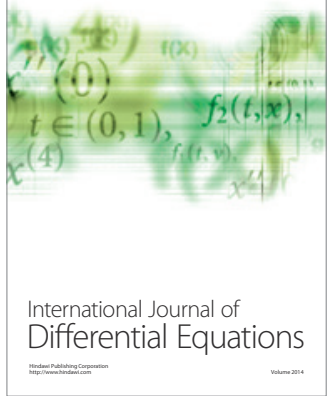
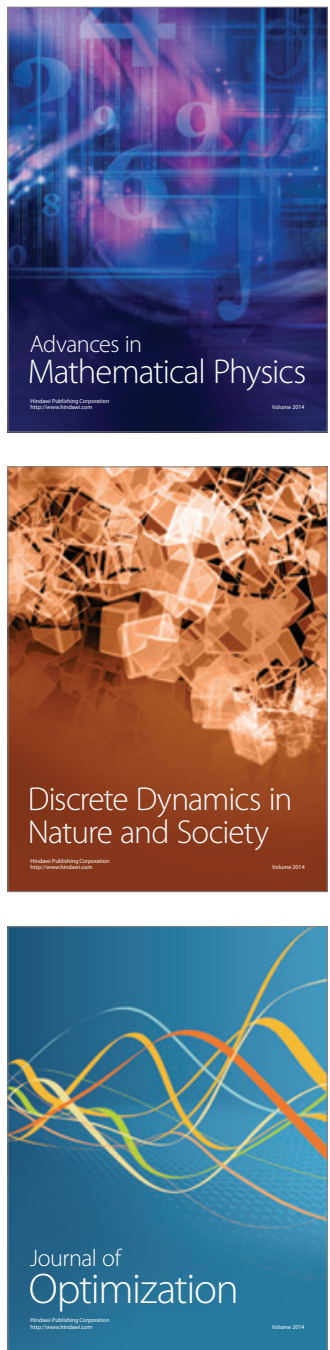